\section{ISBN: 978-90-365-2984-6}

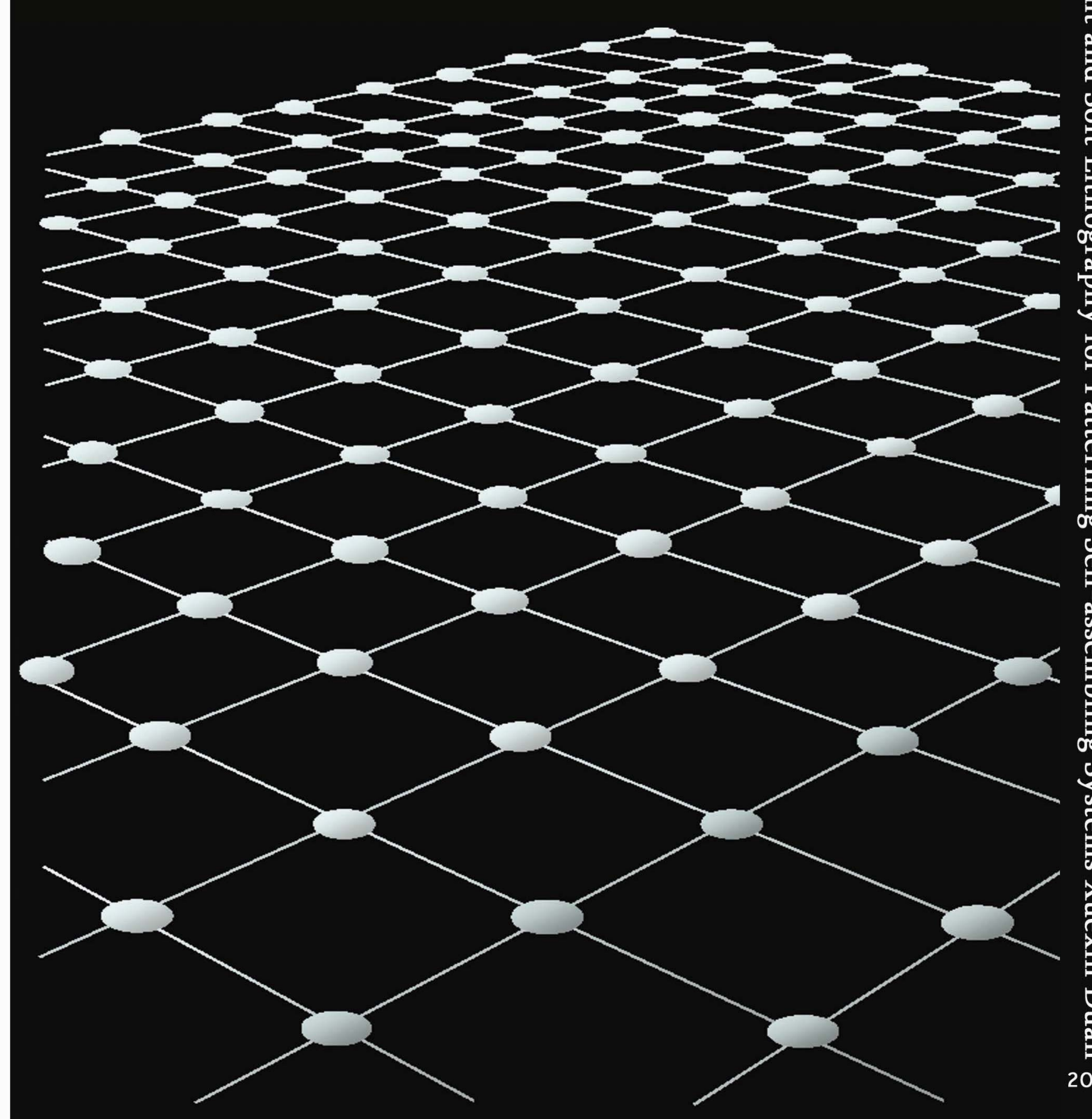

\section{High-Resolution Imprint and Soft Lithography}

for Patterning Self-assembling Systems

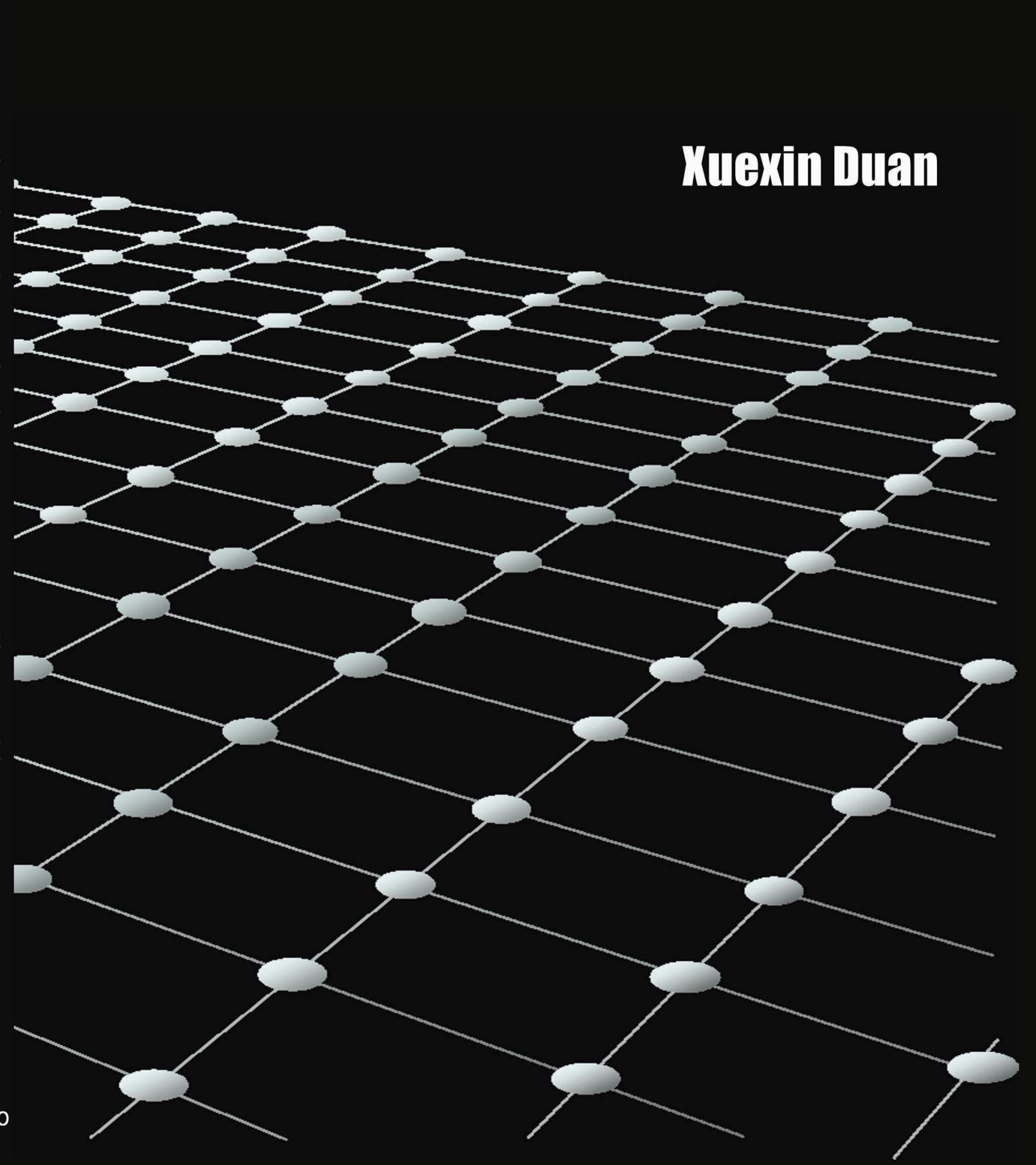

It is my pleasure to invite you to the public defence of my PhD thesis entitled:

High-resolution Imprint and Soft Lithography for Patterning Self-assembling Systems

on Friday, 26th Feb. 2010 at 16.30 in room SP2 of

the Spiegel building at the University of Twente, Einschede, the Netherlands

A party will be held at China Tuin

Oldenzaalsestraat 547 7558 PW, Hengelo (Ov)

Tel; 074-2913252

from 21.30 to 283.30

Xuexin Duan

X.Duan@utwente.nl

Paranimfen

Pieter Moonen

P.F.Moonen@tnw.utwente.nl

Shu-Han Hsu

S.Hsu@tnw.utwente.nl 


\section{HIGH-RESOLUTION IMPRINT AND SOFT LITHOGRAPHY FOR}

PATTERNING SELF-ASSEMBLING SYSTEMS 
The research has been supported by NanoNed, a national nanotechnology program coordinated by the Dutch Ministry of the Economics Affairs (Project No. TMM. 7125).

\section{nano ned}

Publisher: Wöhrmann Print Services, Zutphen, The Netherlands

(C) Xuexin Duan, Enschede, 2010

No part of this work may be produced by print, photocopy or any other means without the permission in writing of the author.

ISBN: 978-90-365-2984-6 


\title{
HIGH-RESOLUTION IMPRINT AND SOFT LITHOGRAPHY FOR \\ PATTERNING SELF-ASSEMBLING SYSTEMS
}

\author{
PROEFSCHRIFT
}

Ter verkrijging van

de graad van doctor aan de Universiteit Twente,

op gezag van de rector magnificus,

prof. dr. H. Brinksma,

volgens besluit van het College voor Promoties

in het openbaar te verdedigen

op vrijdag 26 februari 2010 om 16.45 uur

door

\section{Xuexin Duan}

geboren op 6 juli 1979

te Tianjin, P. R. China 
Dit proefschrift is goedgekeurd door:

Promotoren:

prof. dr. ir. J. Huskens

prof. dr. ir. D. N. Reinhoudt 
To my family 



\section{Table of Contents}

Chapter 1 General Introduction.....................................................1

Chapter 2 Soft Lithography for Patterning Self-assembling Systems................7

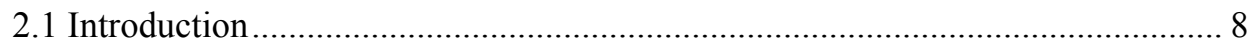

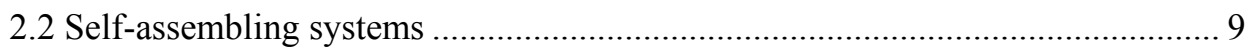

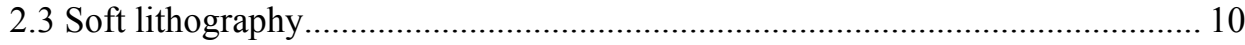

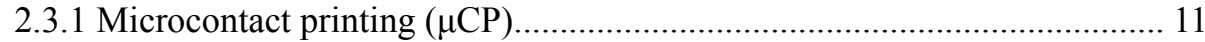

2.3.2 Micromolding injection in capillaries (MIMIC)..................................... 12

2.3.3 Main limitations of soft lithography ……….......................................... 14

2.4 Contact printing of SAMs with high resolution ................................................. 15

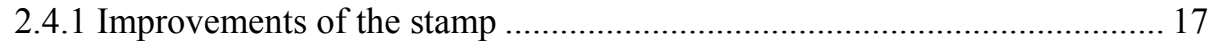

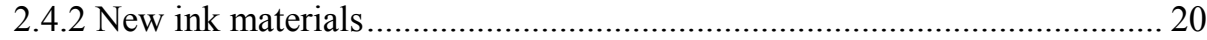

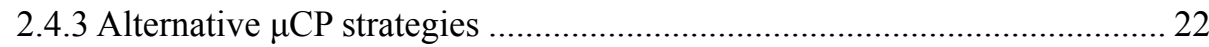

2.5 Soft lithography to pattern assemblies of nanoparticles.................................... 24

2.5.1 Patterning by contact printing................................................................. 24

2.5.2 Patterning by micromolding in capillaries (MIMIC)................................... 30

2.5.3 Patterning by soft lithography with solvent mediation................................ 31

2.6 Soft lithography to pattern supramolecular assemblies........................................... 31

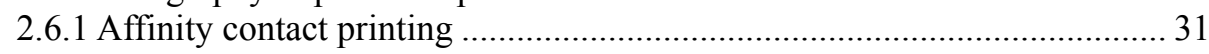

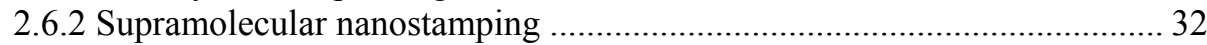

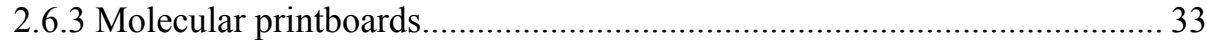

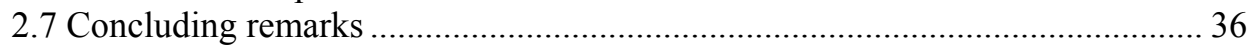

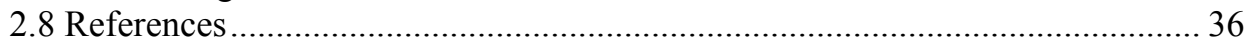

Chapter 3 Bifunctional, Chemically Patterned Flat Stamps for Microcontact Printing of Polar Inks............................................................45

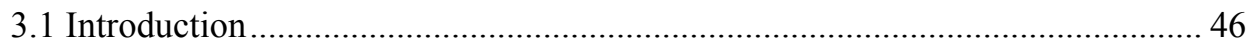

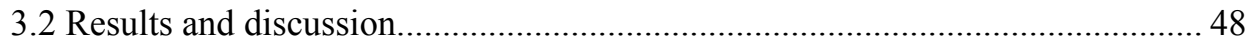

3.2.1 Stamp fabrication and surface characterization ...................................... 48

3.2.2 Microcontact printing using bifunctional flat PDMS stamps ..................... 56

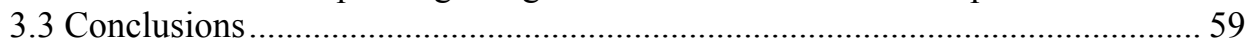

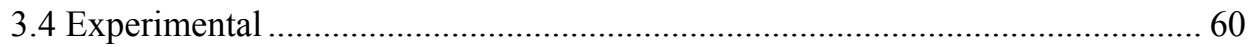

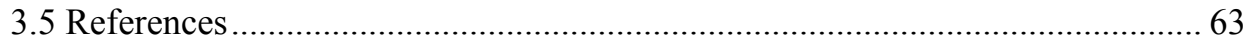

Chapter 4 High-Resolution Contact Printing with Chemically Patterned Flat Stamps Fabricated by Nanoimprint Lithography.................................67 


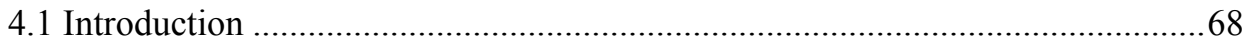

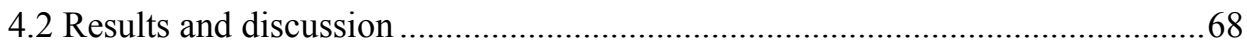

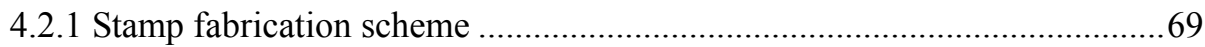

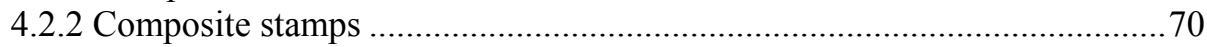

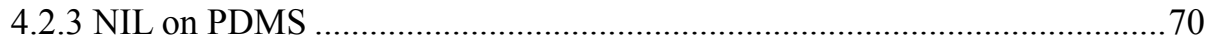

4.2.4 Residual layer removal and pattern transfer..............................................72

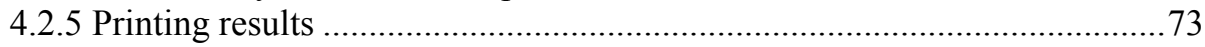

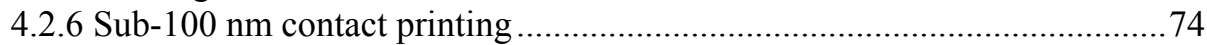

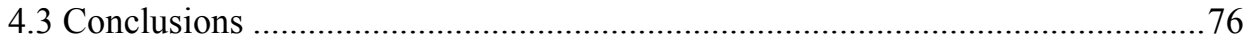

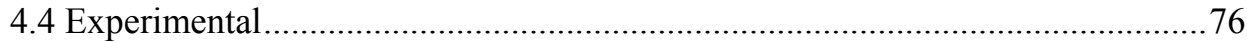

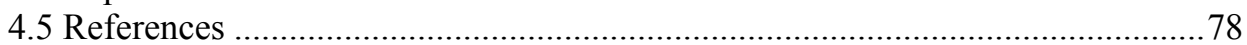

Chapter 5 Nanopatterning by an Integrated Process Combining Capillary Force Lithography and Microcontact Printing..........................................81

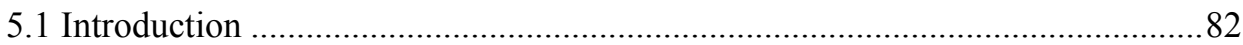

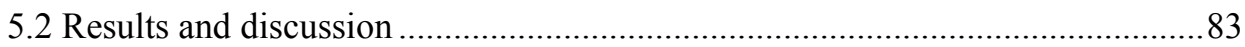

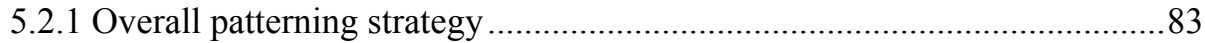

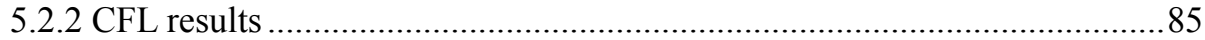

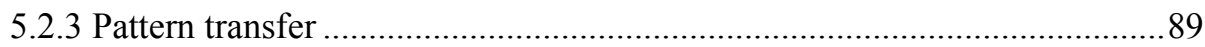

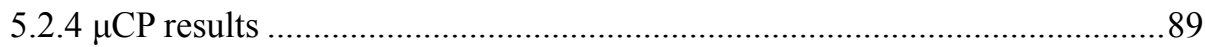

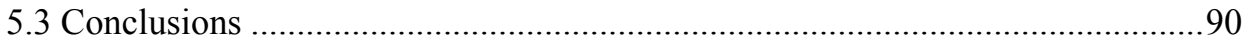

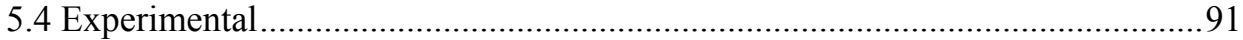

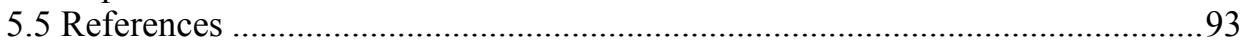

Chapter 6 Large-area Nanoscale Patterning of Functional Materials by Nanomolding in Capillaries......................................................97

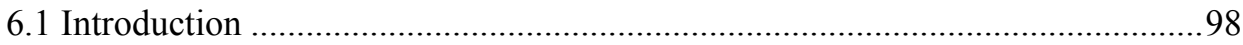

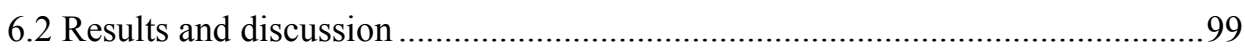

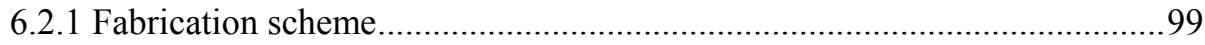

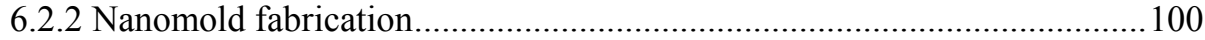

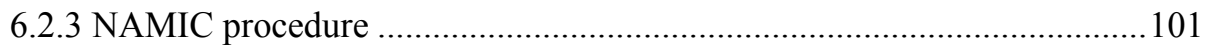

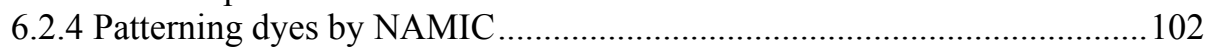

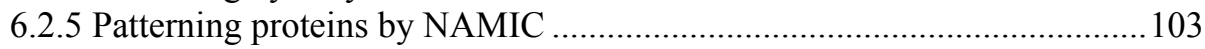

6.2.6 Patterning silica nanoparticles (NPs) by NAMIC .....................................104

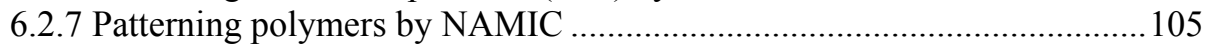

6.2.8 Conductivity measurement of the PEDOT nanowires ………………….....107

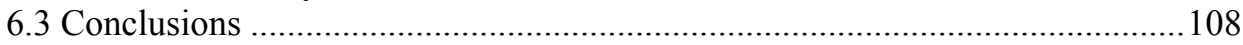

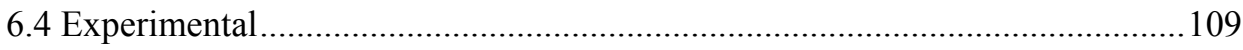

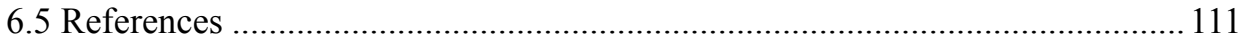


Chapter 7 Assembly, Chemical Modification, and Electrical Characterization of Quasi-1D Gold Nanoparticle Arrays via Nanomolding in Capillaries Combined with Dithiocarbamate Bond Formation...........................................115

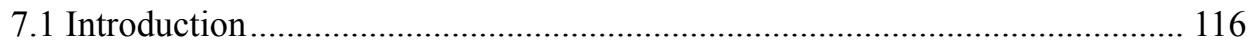

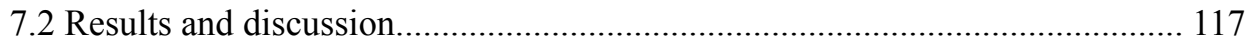

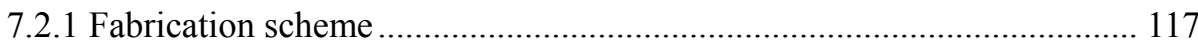

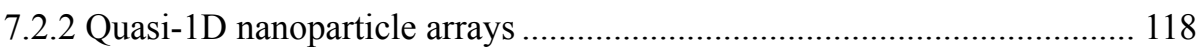

7.2.3 Au nanoparticle arrays as templates for assembly .................................... 122

7.2.4 Electrical properties of the quasi-1D Au nanoparticles arrays.................... 123

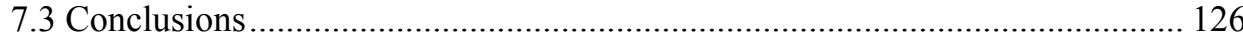

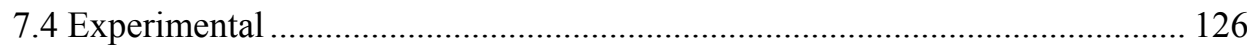

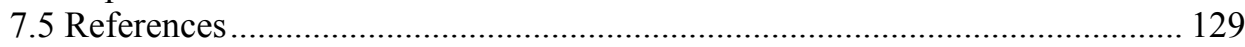

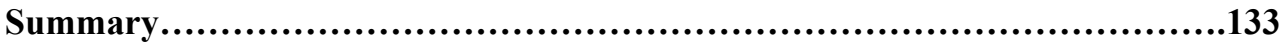

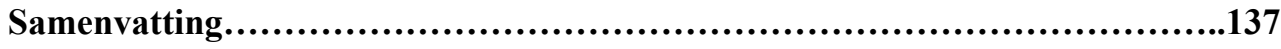

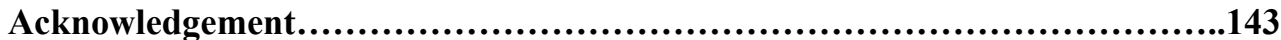





\section{Chapter 1}

\section{General Introduction}

Nanotechnology is a multidisciplinary field of science and technology dealing with the understanding of governing principles of matter at the nanometer scale. Thus, it constitutes the science of objects with the smallest dimensions ranging from a few $\mathrm{nm}$ up to $100 \mathrm{~nm} \cdot{ }^{[1]}$ Objects and structures with these dimensions exhibit peculiar and interesting characteristics. ${ }^{[2,3]}$ The fundamental goal of nanotechnology is the ability to manipulate material properties by controlling their size and shape. ${ }^{[4,5]}$ Nanomaterials synthesized in solution can be well defined and it is now possible to produce all manner of different materials; e.g. metallic, semiconducting, magnetic materials with well-controlled sizes, shapes and surface chemistries. ${ }^{[6]}$ But how do we manufacture useful objects from these nanoscale components? By definition, they are extremely small and difficult to manipulate. To construct anything practical, we must deal with vast numbers of them very rapidly. Nanofabrication fills the gaps between the nanomaterials and functional nanostructures. Two main lines of nanofabrication strategies have evolved: bottom-up and top-down methods.

Bottom-up methods build highly ordered nanostructures from smaller elementary components. The most efficient and applied method is the self-assembly of molecules, surfactants, colloids, block copolymers, etc. The key idea in self-assembly is that the final structure is close to or at thermodynamic equilibrium, and it thus tends to form spontaneously and is prone to error correction. Therefore, it can provide routes to achieve structures with greater order than what can be reached in non-self-assembling systems and thus mainly offers new lithographic possibilities. ${ }^{[7]}$ In another strategy of nanofabrication, "top-down" methods, which consist of a series of lithographic approaches that are based on traditional photolithography, offer arbitrary geometrical designs and superior nanometer- 
level precision, accuracy, and registration. ${ }^{[8]}$ Self-assembly provides simple and low-cost processes to make large-area periodic nanostructures. However, for bulk production of nanostructures, self-assembly has drawbacks, such as the lack of long-range order of the structures, as in most cases they are isotropic and random. The combination of "bottom-up" self-assembly with "top-down" patterned templates can provide new opportunities for development of alternative nanofabrication methods as well as a source of fundamental studies of the self-assembly in confined environments.

The research presented in this thesis contributes to the development of the patterning strategies of the combination of bottom-up and top-down techniques to achieve high resolution (sub-100 nm) patterning. Instead of using conventional lithography techniques (e.g. extreme-UV lithography, e-beam lithography) which generally have the limitations of high capital and operating costs, difficulty of operation, low applicability to several important classes of (bio)organic and organometallic materials, and low applicability to several surface functionalities etc., we have used several unconventional nanofabrication methods (soft lithography, ${ }^{[9]}$ (microcontact printing, micromolding in capillaries), nanoimprint lithography (NIL), ${ }^{[10]}$ capillary force lithography $(\mathrm{CFL}),{ }^{[11]}$ and combinations of these top-down techniques) to pattern or process different self-assembled systems (e.g. self-assembled monolayers, particles, (bio)molecules, polymers, etc.) on surfaces. A focus is on high resolution and high materials versatility especially combined with specific surface chemistry. The self-assembly behavior of different systems in a nano-confined situation has been studied and some applications of these patterned nanostructures have also been discussed. In order to achieve this, the development of chemically patterned flat stamps has been described in Chapters 3-5 to pattern molecules down to sub-100 nm. In Chapters 6 and 7, a hybrid soft stamp with harder, more well-defined features which was fabricated by NIL, was introduced to pattern a large variety of materials (fluorescent dyes, nanoparticles, (bio)molecules, polymers) from their solutions.

Chapter 2 gives an overview of the patterning of self-assembling systems by soft lithography. It starts with a brief introduction of various self-assembling systems and two main soft lithography techniques, microcontact printing $(\mu \mathrm{CP})$ and micromolding in capillaries (MIMIC). Thereafter, recent developments of using $\mu \mathrm{CP}$ and MIMIC for 
patterning self-assembling systems are presented. The selected examples focus on molding high-resolution features in elastomers that are mechanically stable, the need for specific surface chemistry or template design to adsorb molecules, nanoparticles, and biomolecules from solution onto selected areas of the stamp surface, and new strategies to transfer assemblies onto a target surface.

Chapter 3 studies the use of flat stamps to pattern polar inks. Chemically patterned flat PDMS stamps have been shown to result in improved printing resolution in standard microcontact printing because the absence of voids leads to improved stamp stability. In this chapter, the concept of bifunctional, chemically patterned flat PDMS stamps is introduced to patterning hydrophilic ink molecules. $1 \mathrm{H}, 1 \mathrm{H}, 2 \mathrm{H}, 2 \mathrm{H}$ perfluorodecyltrichlorosilane (PFDTS) and 3-(aminopropyl)-triethoxysilane (APTS) were deposited onto flat PDMS surfaces to form bifunctional chemically patterned flat stamps. The PFDTS self-assembled monolayer (SAM) provides an effective barrier to prevent ink transfer, while the APTS SAM areas function as an ink reservoir for polar inks. These stamps were used to transfer polar inks (a thioether-functionalized dendrimer and a fluorescent dye) by microcontact printing.

In Chapter 4, a new method is described to fabricate high-resolution chemical patterns on flat PDMS stamps. Nanoimprint lithography (NIL) was used to fabricate polymer patterns on the flat PDMS substrates at low pressure. The polymer pattern, produced by thermal NIL followed by residual layer removal, acts as a local mask to oxidize the uncovered regions of the PDMS. The chemical patterns were subsequently formed by gas phase evaporation of a fluorinated silane. After removal of the imprint polymer, these stamps were used to transfer alkanethiols as inks to a gold substrate by $\mu \mathrm{CP}$.

In Chapter 5, a nanopatterning process is described by combining capillary force lithography $(\mathrm{CFL})$ and microcontact printing $(\mu \mathrm{CP})$. Flat PDMS was used as the substrate in CFL, and, after chemical functionalization, as the stamp in $\mu \mathrm{CP}$ which increased the resolution of both methods. The polymer patterns, produced by CFL on a thin polymer film on the flat PDMS substrate, acted as a mask to oxidize the uncovered regions of the PDMS. The chemical patterns were subsequently formed by gas phase evaporation of a fluorinated 
silane. After removal of the polymer, these stamps were used to transfer thiol inks to a gold substrate by $\mu \mathrm{CP}$.

Chapter 6 demonstrates a high-resolution soft lithography technique - nanomolding in capillaries (NAMIC). Composite PDMS stamps which consist of flat PDMS, a thin glass layer cured inside the PDMS as a hard support and a thin patterned polymer layer with sub$100 \mathrm{~nm}$ features, were fabricated by nanoimprint lithography (NIL). The fabricated PDMS nanomold can be soft bonded to other substrates to form nanochannels, which can be used to deposit materials through those channels via so-called nanomolding in capillaries (NAMIC). NAMIC has been used to pattern different functional materials such as fluorescent dyes, proteins, nanoparticles, thermoplastic polymers, and conductive polymers at the nanometer scale over large areas. The electrical properties of the nano-patterned conductive polymers have been studied as well.

In Chapter 7, nanomolding in capillaries (NAMIC) combined with dithiocarbamate (DTC) chemistry was used to fabricate sub-50 nm quasi-1D arrays of ultrasmall gold nanoparticles (NPs) over large areas. Owing to the chemical immobilization through the DTC bond, the patterned gold NPs can be further functionalized through thiol chemistry and binding of proteins. The electrical properties of these patterned quasi-1D gold NPs arrays have also been studied.

\section{References}

[1] A. Dowling, Nanosciences and nanotechnologies: opportunities and uncertainties, The Royal Soicety \& The Royal Academy of Engineering, London, July 2004.

[2] M. A. Kastner, Phys. Today 1993, 46, 24.

[3] M. A. Reed, Sci. Amer. 1993, 268, 118. 
[4] C. B. Murray, D. J. Norris, M. G. Bawendi, J. Am. Chem. Soc. 1993, 115, 8706.

[5] S. C. Glotzer, M. J. Solomon, Nat. Mater. 2007, 6, 557.

[6] D. J. Milliron, S. M. Hughes, Y. Cui, L. Manna, J. B. Li, L. W. Wang, A. P. Alivisatos, Nature 2004, 430, 190.

[7] G. M. Whitesides, B. Grzybowski, Science 2002, 295, 2418.

[8] T. Ito, S. Okazaki, Nature 2000, 406, 1027.

[9] Y. N. Xia, G. M. Whitesides, Angew. Chem. Int. Ed. 1998, 37, 551.

[10] S. Y. Chou, P. R. Krauss, P. J. Renstrom, J. Vac. Sci. Technol. B 1996, 14, 4129.

[11] K. Y. Suh, Y. S. Kim, H. H. Lee, Adv. Mater. 2001, 13, 1386. 



\section{Chapter 2}

\section{Soft Lithography for Patterning Self-}

\section{assembling Systems*}

Soft lithography encompasses a family of techniques which employ a soft mold or stamp made by replica molding using a hard master to replicate structures by conformal contact with a substrate to pattern self-assembling systems. It allows the patterning of a wide range of materials and material precursors in a relatively cheap and facile way. This chapter aims to provide an overview of recent advances in patterning self-assembling systems by soft lithography. The selected examples focus on molding high-resolution features in elastomers that are mechanically stable, the need for specific surface chemistry or template design to adsorb molecules, nanoparticles, and biomolecules from solution onto selected areas of the stamp surface, and new strategies to transfer assemblies onto a target surface.

* Part of this chapter has been accepted to publish as a book chapter: X. X. Duan, D. N. Reinhoudt, J. Huskens, "Soft Lithography for Patterning Self-assembling Systems" in Functional Supramolecular Architectures for Organic Electronics and Nanotechnology Ed. Wiley-VCH: Weinheim 2009. 


\subsection{Introduction}

Nanoscience is an important, central theme in fundamental research. It is an emerging science of objects that are intermediate in size between the largest molecules and the smallest structures that can be fabricated by current lithography techniques; that is, the science of objects with the smallest dimensions ranging from a few nanometers to less than 100 nanometers. ${ }^{[1-3]}$ Objects and structures with these dimensions exhibit peculiar and interesting characteristics. ${ }^{[4-7]}$ In addition to their use in the fundamental research field, nanostructures are central to the development of nanotechnologies. In almost all applications of nanostructures, fabrication represents the first and one of the most significant challenges. Two main lines of nanofabrication strategies have evolved: bottomup and top-down methods.

Bottom-up methods build highly ordered nanostructures from smaller elementary components. The most efficient and applied method is the self-assembly of molecules, surfactants, colloids, block copolymers, etc. The key idea in self-assembly is that the final structure is close to or at thermodynamic equilibrium, and it thus tends to form spontaneously and is prone to error correction. Therefore, it can provide routes to achieve structures with greater order than what can be reached in non-self-assembling systems and thus mainly offer new lithographic possibilities. ${ }^{\left[{ }^{[-13]}\right.}$

Self-assembly provides simple and low-cost processes to make large-area periodic nanostructures. However, for bulk production of nanostructures, self-assembly has drawbacks, such as the lack of long range order of the structures, as in most cases they are isotropic and random. In contrast, "top-down" methods, which consist of a series of lithographic approaches, that are based on traditional photolithography ${ }^{[14,15]}$ offer arbitrary geometrical designs and superior nanometer-level precision, accuracy, and registration. The combination of "bottom-up" self-assembly with "top-down" patterned templates can provide new opportunities for fundamental studies of self-assembly behavior in confined environments, as well as a source of innovation for alternative nanofabrication methods. Among them soft lithography, ${ }^{[16-21]}$ which is a series of fabrication techniques that aim to replicate structures on a master by conformal contact with a substrate to pattern self- 
Soft Lithography for Patterning Self-assemling Systems

assembling systems, is most attractive since it allows the patterning of a wide range of materials and material precursors in a relatively cheap and facile way. As such, soft lithography has particularly found its way into the research environment, where rapid prototyping and materials versatility are crucial, and access to high-end photolithography is limited.

This chapter gives an overview of patterning of self-assembling systems by soft lithography. It starts with a brief introduction of various self-assembling systems and two main soft lithography techniques, microcontact printing $(\mu \mathrm{CP})$ and micromolding in capillaries (MIMIC). Thereafter, recent developments of using $\mu \mathrm{CP}$ and MIMIC for patterning self-assembling systems will be presented. A main focus is on advances in resolution.

\subsection{Self-assembling systems}

Self-assembly is the spontaneous organization of molecules or objects into stable, welldefined structures by noncovalent forces. It has been the focus of vast research efforts in the last four decades. These efforts have produced a solid foundation of understanding of the physics and chemistry of self-organizing processes. ${ }^{[22]}$

Self-assembly starts with the smallest units - molecular assembly. In molecular selfassembly, multiple weak, reversible interactions, based on, for example, hydrogen bonding and Van der Waals forces, are the driving forces to assemble individual molecular subunits into stable aggregates. The assembled structure typically represents a thermodynamic minimum that results from equilibration of these interactions. There are hundreds of examples of systems that based on the self-assembly of molecules, such as liquid crystals, micelles, and lipid membranes. Although many of the studies in self-assembly have focused on molecular components, the domain of self-assembly is not limited to the molecular level, but extends to structural organizations on various length scales. Energy minimization remains the key motivation for self-assembly at these scales. Self-assembly of larger components, e.g., nanoparticles, biomolecules, block polymers, etc., shows lots of promise and is extremely important in the emerging fields of nano-structured materials, bio-inspired templating, DNA computing and new developments in microelectronics. ${ }^{[23]}$ 
In general, self-assembly can be classified in: (i) nontemplated self-assembly, where individual components interact to produce a larger structure without the assistance of external forces or spatial constraints, and (ii) templated self-assembly, where individual components interact with one another guided by an external force or within spatial constraints. In this review we will focus on the recent advances in templated self-assembly, in particular examples patterned by soft lithography, and evaluate their application for the nanofabrication of $1 \mathrm{D}, 2 \mathrm{D}$, and $3 \mathrm{D}$ materials.

\subsection{Soft lithography}

Photolithography is exclusively being used for the fabrication of microelectronic devices. ${ }^{[14,15]}$ However, photolithography has limitations when targeting the patterning of self-assembling systems: (i) it requires expensive instruments and facilities with high capital investment, (ii) the pattern resolution is limited by optical diffraction and obtaining submicron patterns requires extensive technology up-grading, (iii) it is not suitable for patterning all types of polymers as only photosensitive resist materials (photoresists) can be directly patterned, (iv) it requires harsh processing conditions like exposure to UV-radiation and chemical etching, therefore, it is not suitable for patterning sensitive materials. Some of the limitations of photolithography, particularly the resolution limit, can be overcome by using next-generation lithography, e.g. deep UV and extreme UV photolithography, ${ }^{[24,25]}$ soft X-ray lithography, ${ }^{[26]}$ electron-beam writing, ${ }^{[27]}$ and ion-beam lithography. ${ }^{[28]}$ However, all of these state-of-the-art lithographic techniques have high costs and low accessibility. There is a large demand for low-cost and large-area manufacturing techniques, both for real applications in actual devices, but in particular for rapid prototyping in research environments. Soft lithography, which is a collective term for a number of nonphotolithographic techniques, fulfills these requirements, to some extent, by removing the need for cleanroom facilities. Furthermore, it allows the direct patterning of a wide range of materials.

Soft lithography encompasses a family of techniques which employ a soft mold or stamp made by replica molding a soft polymer using a hard master. Varying the way in which the molds are used results in different techniques: microcontact printing $(\mu \mathrm{CP})$, ${ }^{[29]}$ 
Soft Lithography for Patterning Self-assemling Systems

micromolding in capillaries (MIMIC), ${ }^{[30,}{ }^{31]}$ replica molding (REM) ${ }^{[32]}$ microtransfer molding $(\mu \mathrm{TM}),{ }^{[33]}$ and solvent-assisted micromolding (SAMIM) ${ }^{[34]}$ The present review will focus on the first two methods, since they offer the possibilities to pattern selfassembling systems at solid state or from their solutions. Emphasis is put on new developments which provide access to difficult length scales, i.e., between a few tens and hundreds of nanometers.

\subsubsection{Microcontact printing $(\mu \mathrm{CP})$}

Whitesides et al. invented the microcontact printing $(\mu \mathrm{CP})$ process at the beginning of 1990s. The general procedure of the $\mu \mathrm{CP}$ is remarkably simple (Figure 1). It works by the creation of a flexible, polymeric stamp with patterned reliefs (typically made from poly(dimethylsiloxane), PDMS) and by dipping the stamp in an alkanethiol 'ink'. Once the stamp has been inked and dried, it is then briefly pressed against a gold (or other thiolcompatible) substrate via conformal contact, and the alkanethiol molecules are transferred from the polymer to the substrate and self-assemble into a self-assembled monolayer (SAM) in the contact areas. The bare gold regions can either be etched, used directly, or backfilled with a different adsorbate. In the latter way, a binary-component SAM is formed which can act as a template for the selective deposition of specific molecules (e.g. proteins).

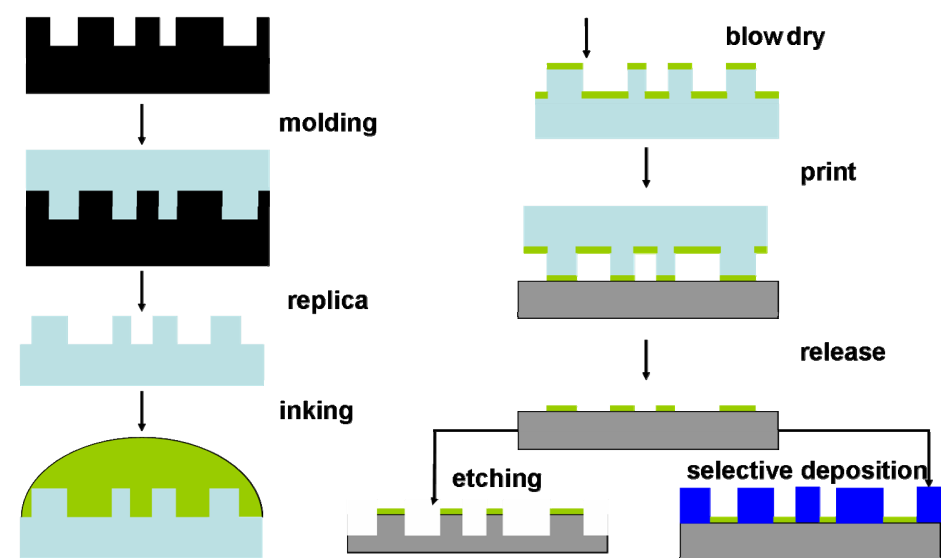

Figure 1. Schematic representation of the microcontact printing ( $\mu C P)$ process. 
$\mu \mathrm{CP}$ as a method of patterning SAMs has grown in popularity due to the ease of fabrication of the printing tools, relatively high spatial resolution of the features produced and large printing capacities. ${ }^{[20,35-37]}$ The low costs and simplicity of the technique have inspired the interest in creating smaller patterns with higher edge resolution, and in broadening the versatility of the technique. Although initially mainly used as a method for patterning self-assembled alkanethiol monolayers on gold surfaces, ${ }^{[37-39]} \mu \mathrm{CP}$ has been extended to alkylsilanes on silicon oxide, ${ }^{[40]}$ and this has resulted in numerous biotechnology applications, such as the patterned growth of a variety of cells and the fabrication of microarrays for biosensor purposes. The range of ink molecules has been extended from alkylsilanes and alkanethiols to various particles and organic molecules with higher molecular weights, ranging from Langmuir-Blodgett films ${ }^{[41]}$ to $\mathrm{DNA}^{[42,43]}$ and proteins. ${ }^{[44]}$ Nowadays, $\mu \mathrm{CP}$ has been recognized as a remarkable surface patterning technique and has triggered enormous interest from the surface science community, as well as from engineers and biologists.

\subsubsection{Micromolding injection in capillaries (MIMIC)}

Micromolding in capillaries (MIMIC) is a simple and versatile soft-lithographic method that forms complex microstructures on both planar and curved surfaces. It was introduced by Whitesides and coworkers in 1995. ${ }^{[31]}$ MIMIC, which scheme is shown in Figure 2, can be considered a precursor of micro- and nanofluidics, and it can be used to pattern many soluble materials.

In a typical MIMIC scheme, a soft elastomeric mold usually made from PDMS with parallel protrusions is placed in contact with a smooth surface, so that the grooves form channels (capillaries). When a solution is poured at the open end of the channels, the liquid spontaneously fills the channels under the effect of capillary pressure. As the solution volume gradually shrinks because of solvent evaporation, the capillary forces drive the formation of a meniscus under the roof of the stamp channels. After the complete evaporation of the solvent, the stamp is gently removed leaving the patterns on the surface.

Depending on the concentration of the solution, two kinds of patterns can be obtained. In the high concentration regime, if the solution reaches supersaturation when the 
microchannel is still full of solution, the pattern replicates the size of the microchannel. In the dilute regime, when the solution reaches supersaturation when most of the solvent has evaporated and the volume of the residual solution is not enough to fill the channel completely, the solution tends to accumulate on the boundaries of the channel, giving rise to some defects in the microstripes or to split lines.

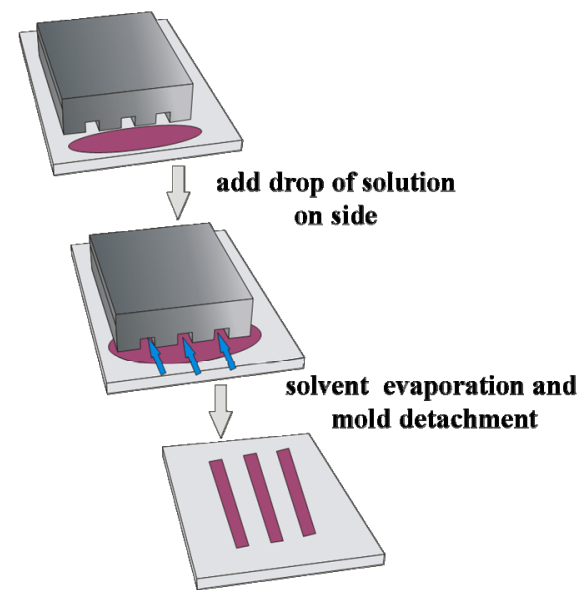

Figure 2. Schematic representation of the micromolding in capillaries (MIMIC) process.

The capability of MIMIC has been demonstrated by the fabrication of patterned structures and devices from a variety of functional materials at the micrometer and nanometer length scales. Although MIMIC was used initially without solvent, ${ }^{[30]}$ that is, using a prepolymer instead of a solution, the most recent applications employ solutions of functional molecules, for which the only requirement is that the solvent does not swell the polymeric stamp.

Capillarity is the main driving force in MIMIC for filling the channels. During the solvent evaporation, the self-organization of the solute enters into play when the solution reaches supersaturation, so that spatially organized nanodots, wires, or crystallites can be fabricated. It proved that MIMIC is an excellent tool both for patterning and studying selfassembling systems. 


\subsubsection{Main limitations of soft lithography}

The key element in soft lithography is the use of a patterned elastomeric stamp that mediates intimate contact between the elastomeric stamp and the substrate. The definition of this intimate or 'conformal' contact in high-resolution printing goes beyond the contact between the asperities of two flat, hard surfaces. Adhesion forces mediate this elastic adaptation, and even without the application of external pressure, an elastomer can spontaneously compensate for some degree of substrate roughness, depending on the materials properties. ${ }^{[35]}$

The elastomer stamp that is used in soft lithography is most commonly poly(dimethylsiloxane) (PDMS). This is favored because it is commercially available, resistant to many types of chemicals and $\mathrm{pH}$ environments, optically transparent, and nontoxic. PDMS stamps can be reused many times without noticeable degradation. The material cures under moderate conditions and is easily removed from surfaces, making it amenable to patterning complex structures on delicate or non-planar surfaces.

The use of a soft polymer is also at the origin of the main problems of soft lithography. Deformation of the stamp during stamp removal from the template and during the contacting of the substrate limits the resolution of the patterning. Such deformations are illustrated in Figure 3. The height of the features divided by their lateral dimensions defines the aspect ratio of a pattern. When the aspect ratio is high, buckling and lateral collapse of the PDMS features can occur, while at low aspect ratios roof collapse is possible. ${ }^{[45]}$ Any deformation of the stamp will affect the printed pattern and decrease reproducibility. These phenomena are enhanced when the sizes of the corrugations reach submicron or nanoscale. 


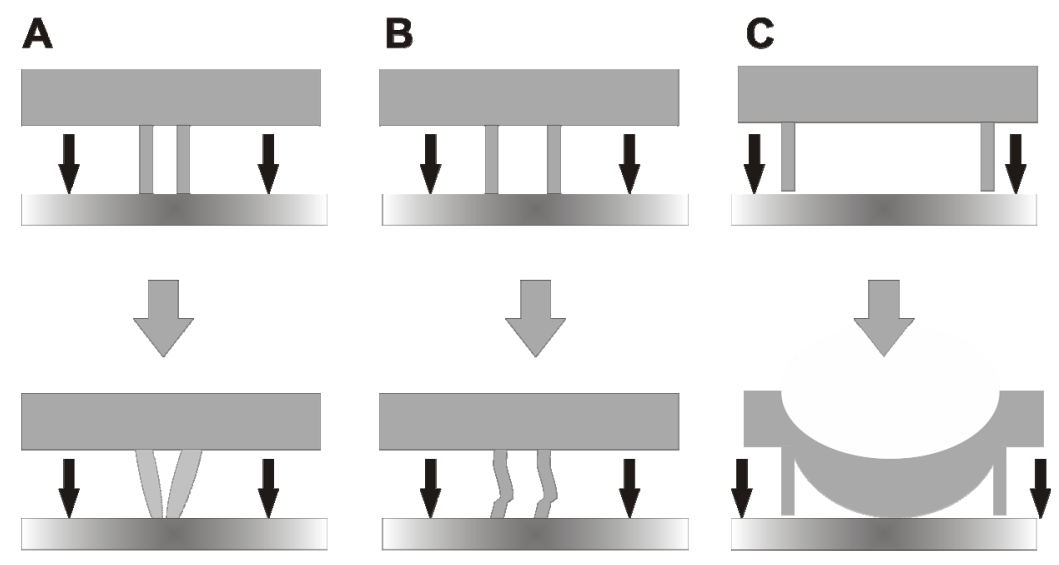

Figure 3. The most commonly observed stamp deformations: pairing (A), buckling (B), and roof collapse $(C)$.

Beside the stamp deformation, there are some other major drawbacks when using PDMS as the stamp or mold in soft lithography. For example, the patterns can be contaminated with unpolymerized low molecular weight siloxanes co-transferred from the stamp and thus decrease the pattern quality. ${ }^{[46-49]}$ Swelling and distortion of the stamp may occur with certain solvents, especially in the case of nonpolar solvents, and may result in patterns with increased sizes and pattern defects. ${ }^{[50-52]}$

These problems have limited the size of the patterns that can be attained by soft lithography to the $>500 \mathrm{~nm}$ scale. In recent years, efforts have been made to shrink the size of the patterns to nanoscale. To overcome the obstacles described above, the optimization of stamp materials and improvements of the patterning conditions are crucial. Furthermore, new patterning strategies combined with self-assembling systems have enabled the possibility of patterning with nanoscale dimensions through microcontact printing or micromolding in capillaries.

\subsection{Contact printing of SAMs with high resolution}

Self-assembled monolayers (SAMs) constitute an important class of self-assembling systems. SAMs are ordered molecular assemblies formed by the adsorption of an active surfactant to a solid surface. The order in these two-dimensional systems is produced by a 
spontaneous chemisorption process at the interface, as the system approaches equilibrium. ${ }^{[53]}$ SAMs have shown potential applications in the control of wettability, biocompatibility, and corrosion resistance of the surfaces of a wide range of materials. ${ }^{[54,55]}$ Among all SAM systems, the assembly of sulfur-containing molecules, such as alkanethiols and dialkyldisulfides on noble metal surfaces (especially on gold) have been the most widely studied. The assembly is held together by the bonds between the sulfur headgroups and the gold surface as well as by Van der Waals interactions between the neighboring hydrocarbon chains. The surface chemistry can be entirely defined by the nature of the functional group at the end of the long-chain alkanethiols used. The facile formation of these self-assembled monolayers has opened a general route to molecular-level control over surface order and chemistry.

Patterning SAMs offers the possibility to form SAMs in predetermined spatial distributions and has thus created in applications for nanotechnology. ${ }^{[53,56]}$ Patterning SAMs in the plane of the surface has been achieved by a wide variety of techniques. ${ }^{[57]}$ Among them, microcontact printing $(\mu \mathrm{CP})$, which relies on the contact of a rubber stamp inked with alkanethiols with a substrate, offers a most interesting combination of convenience and new capabilities. Contact time are typically in the order of seconds, but despite these short contact times, high-quality SAMs are formed that differ very little from the crystalline SAMs formed from solution over hours as demonstrated by scanning tunneling microscopy (STM) studies. ${ }^{[58]}$

As simple as $\mu \mathrm{CP}$ is, however, it has been very difficult to print SAMs with sub-500 $\mathrm{nm}$ lateral resolution. Besides the stamp deformation problem, which is mentioned in section 2.3.3, another important issue is the ink diffusion problem. In $\mu \mathrm{CP}$, the successful transfer of molecules to the substrate is typically achieved by ink diffusion of absorbed molecules from the PDMS bulk to the surface. However, an excess of ink results in the uncontrolled transfer and spreading of the ink molecules. Delamarche et al. have studied in detail the ink transfer mechanism during the microcontact printing process. ${ }^{[59]}$ They proved that ink transfer to the noncontact areas can happen because of ink diffusion through the stamp, ink spreading, surface diffusion, and vapor phase deposition. All those issues limit 
Soft Lithography for Patterning Self-assemling Systems

the lateral resolution of the contact printing of SAMs. Here, examples of recent advances in microcontact printing to achieve high resolution will be reviewed.

\subsubsection{Improvements of the stamp}

\subsubsection{Hard PDMS and composite stamps}

In order to solve stamp deformation with the conventional (Sylgard 184 system) PDMS, the IBM group has identified a commercially available Si-based elastomer that has a relatively high compression modulus. ${ }^{[60]}$ This material (hard-PDMS, or h-PDMS) is prepared from trimethylsiloxy-terminated vinylmethylsiloxane-dimethylsiloxane (VDT-731; Gelest) and methylhydrosiloxane-dimethylsiloxane (HMS-301; Gelest) copolymers. The hPDMS system has cross-linkers that have relatively short lengths as compared to those in the Sylgard 184 system thus it has a relatively high modulus $(\approx 9 \mathrm{MPa})$. With this "hard" PDMS, the contact printing of structures down to $80 \mathrm{~nm}$ was achieved. Choi et al. ${ }^{[61,62]}$ designed a new stiffer PDMS stamp that incorporates a rigid urethane methacrylate crosslinker into the PDMS polymeric network. It has a modulus of $4 \mathrm{MPa}$ which is higher than conventional Sylgard $184(\approx 2 \mathrm{MPa})$ but lower than h-PDMS. It performs better in printing in many respects than the commercially available materials. In addition, the physical toughness and modulus of this material can be adjusted by controlling the cross-linking density. Moreover, its photocurability allows the elements to be patterned by exposure to light through a mask. Suh et al. ${ }^{[63]}$ developed a PDMS stamp with corrugations that were reinforced by chemical vapor deposition-induced polymerization of poly(p-xylylene) on the side walls of the structure. It also proved to be a good candidate for the fabrication of deformation-proof stamps.

Drawbacks associated with the relatively brittle nature of h-PDMS and the thermal curing requirements of the material remain. In order to overcome the brittle behavior of $\mathrm{h}$ PDMS, the IBM group also tested several composite stamp designs that used a rigid support (glass or quartz) and a thin h-PDMS top layer. ${ }^{[60]}$ These designs included multilayer stamps (a thin layer of h-PDMS on a slab of Sylgard 184 system attached to a quartz plate) and two-layer stamps (thin layers of h-PDMS or Sylgard 184 molded to glass foils). ${ }^{[60,64]} \mathrm{A}$ 
stamp, consisting of a thin $(30 \mu \mathrm{m})$ layer of h-PDMS supported on a flexible glass foil (100 $\mu \mathrm{m}$ thick), conformed the best to uneven substrate topologies with minimal distortion over large areas. Whitesides et al. ${ }^{[65]}$ modified these fabricated composite stamps using a Sylgard 184 slab to support a thin, stiff h-PDMS top layer. The smallest features that have been replicated using such composite stamps were $50 \mathrm{~nm}$.

It is important to note that by increasing the stiffness of the elastomeric material, higher-resolution patterns can be produced; however, stiffer materials decrease the conformability of the stamp or mold, thereby reducing the contact between the stamp and the substrate and causing defects in the patterns. In addition, stiffer materials limit the versatility of the patterning technique since these cannot be used with non-planar substrates. Therefore, an acceptable balance must be achieved between the conformability and stiffness of the material in order to produce patterns reproducibly.

\subsubsection{Other stamp materials}

Apart from PDMS, an enormous variety of other polymeric elastomers is available that derive their elastomeric character from, for instance, self-aggregation of thermoreversible, nanosized structures within the bulk of the polymers. Typically, these elastomers are classified as thermoplastic elastomers. The properties of these elastomers with respect to stiffness can be tuned, to a large extent, by a proper selection of their chemical structure, composition, and processing conditions while preserving their intrinsic toughness. Csucs et $a l .{ }^{[66]}$ reported the use of polyolefin plastomers (POPs) for the printing of protein and block copolymer patterns. It was shown that in the submicrometer range (submicrometer structures with micrometer separations), a much higher printing quality is achievable with the POPs compared to regular PDMS. This fact is probably due to the higher bulk modulus of the POP stamps. Trimbach et al. ${ }^{[67]}$ studied two commercially available thermoplastic block copolymer elastomers (poly(styrene- $b l$-butadiene- $b l$-styrene)) and (poly(styrene- $b l$ ethylene-co-butylene- $b l$-styrene)) with high stiffness as stamp materials for microcontact printing. They showed that the thermoplastic elastomers possess a high modulus and toughness in comparison to PDMS, and consequently the stamp deformation during printing is decreased. A UV-curable stamp material, a poly(urethane acylate) based on a 
Soft Lithography for Patterning Self-assemling Systems

functionalized prepolymer with acrylate groups for cross-linking and different monomeric modulators, was developed in Lee's group. ${ }^{[68]}$ By varying the modulator, the mechanical properties of the stamp could be tuned. A series of materials based on perfluoropolyether (PFPE) has also been developed as stamp materials for high resolution printing. ${ }^{[69-71]}$ The fluoropolymer, which is liquid at room temperature, can be crosslinked under UV light to yield elastomers with an extremely low surface energy $\left(\approx 12 \mathrm{mN} \mathrm{m}^{-1}\right)$. A major advantage of PFPE-based materials is that they are solvent resistant and chemically robust and therefore swell much less than PDMS when exposed to most organic solvents. This property expands the range of materials that can be patterned effectively. Also, unlike PDMS, PFPEs eliminate the surface functionalization step that is often required to avoid adhesion to oxides (e.g., $\mathrm{SiO}_{2}$ on $\mathrm{Si}$ wafers) during the casting and curing steps used to make the patterning elements. These PFPE-based materials allow the replication of sub-100 $\mathrm{nm}$ sized features with no indications of limits when going to even smaller sizes.

\subsubsection{Flat stamps}

The mechanical issues are a direct consequence of the inclusion of topographical voids as the transport barriers. In principle, a flat stamp can solve many or all stamp stability issues. Geissler et al. ${ }^{[72]}$ first used a planar PDMS stamp to print chemical patterns onto a substrate. Delamarche et al. ${ }^{[73]}$ have shown that flat PDMS stamps can be patterned by a combination of surface oxidation in an oxygen plasma using a mask and subsequent stabilization of the hydrophilized areas by reaction with a poly(ethylene oxide) silane. These stamps have been used for the selective deposition and subsequent patterned transfer of proteins from the stamp surface. Later they developed a method by using flat stamps to pick up proteins from a hard nano-template, thus creating sub-100 nm protein patterns and called this approach subtractive lithography. ${ }^{[7]}$

In our group, we have introduced the concept of flat, chemically patterned stamps for the $\mu \mathrm{CP}$ of regular thiol inks. ${ }^{[75]}$ Stamp functionalization was achieved by local oxidation of a flat piece of PDMS through a mask, followed by adsorption of a fluorinated silane, $1 \mathrm{H}, 1 \mathrm{H}, 2 \mathrm{H}, 2 \mathrm{H}$-perfluorodecyl-trichlorosilane (PFDTS). It was found that this silane forms densely packed SAMs on oxidized PDMS, which constitutes an effective barrier to prevent 
ink transfer, while the rest of the surface allows the diffusion of ink molecules from the bulk of the PDMS to the gold substrates (Figure 4).
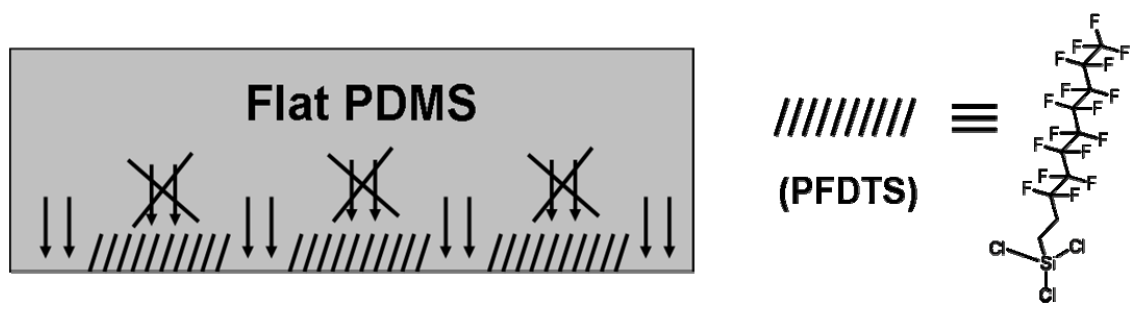

Figure 4. Schematic representation of printing thiols with flat stamps: the $1 \mathrm{H}, 1 \mathrm{H}, 2 \mathrm{H}, 2 \mathrm{H}$ perfluorodecyltrichlorosilane-covered area acts as an ink barrier, while the non-covered PDMS transfers the thiols to the gold substrate.

The flat stamp design effectively solves the stamp stability issues, and sub-micrometersized features were successfully printed using these chemically patterned flat stamps. However, the main difficulty is the fabrication of the chemical barriers on the stamp's surface as the pattern size is limited by the mask used for the selective oxidation. Other methods have been used to fabricate chemical patterns on flat PDMS surfaces, targeting higher resolutions. Zhang recently reported the use of dip-pen nanolithography (DPN) to directly write sub-100 nm chemical patterns on flat PDMS. ${ }^{[76]}$ The limitations of this method are the longer writing time and the difficulties in writing on the flexible substrates.

\subsubsection{New ink materials}

Among all the limitations of $\mu \mathrm{CP}$, the diffusion of the ink molecules during and after the printing process is a significant problem in getting high-resolution patterns. When feature sizes are smaller than $500 \mathrm{~nm}$, ink diffusion compromises the final resolution. The use of a heavy ink allows the diffusion zone to be reduced to $100 \mathrm{~nm}$ for immersion inking and less than $50 \mathrm{~nm}$ for contact inking of the stamp. ${ }^{[35]}$ Bass and Lichtenberger ${ }^{[77]}$ showed that a higher molecular weight alkanethiol, such as octadecanethiol or eicosanethiol, diffuses less on a gold surface compared to hexadecanethiol and exhibits concomitantly better printing results. Longer chain thiols (longer than eicosanethiol) tend to show more disordered layers on gold, thus leading to poorer etch performance. 
Soft Lithography for Patterning Self-assemling Systems

Other ways of improving the resolution of the microcontact printed patterns include the use of inks heavier than alkanethiols (Figure 5). Liebau et al. ${ }^{[78]}$ investigated some heavy molecular weight thioether derivatives as inks in microcontact printing. Poly(amidoamine) (PAMAM) dendrimers have been used as inks to be transferred to silicon substrates, ${ }^{[79]}$ palladium, ${ }^{[80]}$ or gold substrates. ${ }^{[81]}$ Sub-100 $\mathrm{nm}$ resolution has been achieved by these dendrimer inks. In these studies, the ink molecules did not show diffusion on the patterned surface because of their high molecular weight, thus leading to a more faithful transfer of the inks.

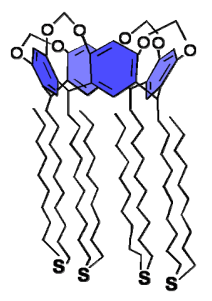

a

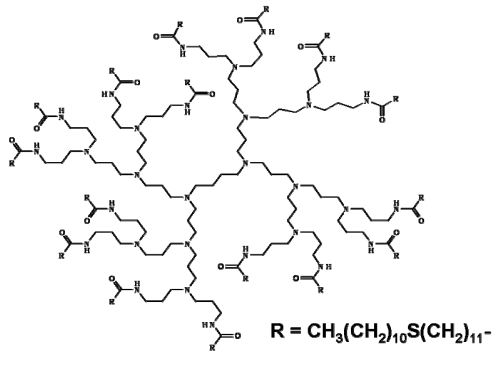

b

Figure 5. Some examples of heavyweight inks: a calix [4] arene thioether derivative (a) and thioether-modified dendrimer (b).

However, heavy inks also have their own limitations, such as longer printing times, less ordered monolayers, and the tendency to crystallize at the surface of the stamp leading to contamination problems. In order to achieve high resolution printing there must be a balance between the molecular weights of the inks, the printing time, the inking time, the amount of the inks, and other parameters. Recently Balmer et al. studied in detail the diffusion behavior of several alkanethiols in PDMS stamps. ${ }^{[82]}$ Their results showed that the ink transport through the PDMS stamps follows Fick's laws of diffusion. The diffusion coefficient was also calculated for three different alkanethiols. These studies constitute the basis of future optimizations of the printing conditions. 
Beside using molecules as inks, a few nanometer thin metal layers evaporated on the stamp surface could be transferred onto substrates. ${ }^{[83,84]}$ The process, described by Rogers and coworkers, is called nanotransfer printing (nTP) and uses an adhesive SAM on the substrate to stick to the metal layer and to promote the release of the layer from the stamp when the stamp is removed. The technique can generate complex patterns without the risk of surface diffusion and edge disorder.

\subsubsection{Alternative $\mu \mathrm{CP}$ strategies}

\subsubsection{High-speed $\mu \mathrm{CP}$}

After a series of studies on understanding ink transport and diffusion mechanisms, ${ }^{[59,82]}$ a breakthrough in the timescales involved in stamp contact and monolayer formation was reported in a recent paper on high-speed microcontact printing by the IBM group. ${ }^{[85]}$ In a careful analysis of the mechanics of the printing procedure and a numerical diffusion simulation of the ink transfer, they concluded that $\mu \mathrm{CP}$ can be performed up to three orders of magnitude faster than previously reported. For example, a printing time of $3 \mathrm{~ms}$ using a concentration of $16.6 \mathrm{mM}$ of hexadecanethiol ink is sufficient to create same quality pattern replication in printed and etched gold patterns. These recent results demonstrate that there is a well-defined processing window, in which the combination of ink concentration and contact time yield perfect SAMs (as judged from etch resistance, thus also indicating the formation of monolayers with crystalline order), but where surface diffusion, diffusion through the vapor phase, ink depletion, and stamp distortion are all avoided (Figure 6). This major improvement illustrates the possibilities to scale down the lateral resolution of $\mu \mathrm{CP}$ and indicates that $\mu \mathrm{CP}$ could become a commercially attractive micro(nano)fabrication technology. 
Soft Lithography for Patterning Self-assemling Systems

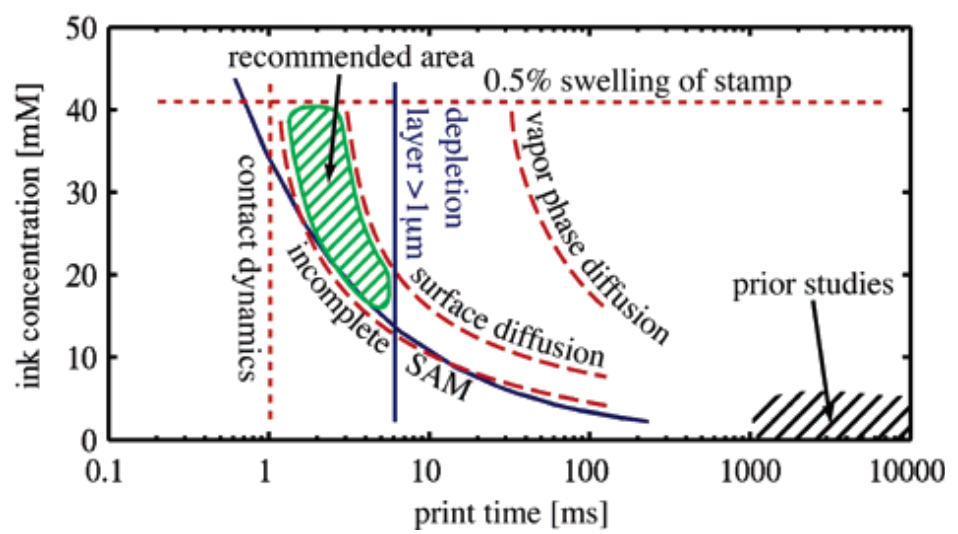

Figure 6. High-speed $\mu$ CP process window as a function of ink concentration and contact time.

\subsubsection{Catalytic printing}

In catalytic printing, the PDMS stamp used can be made catalytically active either by chemical surface modification or UV treatment in order to induce a chemical reaction with the substrate upon contact. In such a catalytic approach, no ink is needed, and therefore the lateral resolution-reducing effect of surface spreading is efficiently eliminated.

In our group, surface-oxidized PDMS stamps have been used to hydrolyze silyletherprotected SAMs in the areas of contact, thus forming patterned SAMs. Because there was no ink flow from the stamp to the surface, high edge resolution (below $60 \mathrm{~nm}$ ) was obtained ${ }^{[86]}$ A similar study used plasma-oxidized flat PDMS to promote coupling between amino-terminated SAMs and N-protected amino acids under nanoscale confinement by contact printing. ${ }^{[87]}$

Park et al ${ }^{[88]}$ have developed a printing method to directly transfer the contact surface of the PDMS stamp on a substrate via a UV light-induced surface bonding between the stamp and substrate. First a patterned PDMS stamp was prepared which was activated by exposure to UV/ozone and brought into contact with the substrate. Because of the UV/ozone treatment, an irreversible adhesion reaction occurred between the PDMS and the substrate. After the release of the stamp, PDMS patterns were formed on the substrate. The 
PDMS patterns can be used not only as resists for selective wet etching, but also as templates to selectively deposit $\mathrm{TiO}_{2}$ thin films.

Toone and coworkers used piperidine-functionalized poly(urethane acrylate) stamps to promote the catalytic cleavage of the 9-fluorenylmethoxycarbonyl amino-protecting group. ${ }^{[89]}$ With this inkless method, sub-micrometer patterns were created by selective deprotection of (9H-fluoren-9-yl)methyl-11-mercaptoundecylcarbamate SAMs on gold. Later, they extended the use of catalytic $\mu \mathrm{CP}$ to biochemical substrates by immobilizing exonuclease enzymes on biocompatible poly(acrylamide) stamps and created patterned DNA both on glass and gold surfaces. ${ }^{[90]}$

\subsection{Soft lithography to pattern assemblies of nanoparticles}

Nanoparticles (NPs) are most versatile tools for the construction of new and advanced tunable materials. ${ }^{[91]}$ Their intriguing properties make them useful in a wide range of applications in optics, chemical sensors, data storage, etc. ${ }^{[92-94]}$ A prerequisite for future applications using NPs as functional entities is the control over their positions and arrangement on a surface. Doing so with conventional microfabrication techniques is difficult, and it is often time-consuming and inefficient. Soft lithography, in contrast, offers the possibility to pattern NPs in a relatively easy way. Patterning using imprint lithography has been reviewed before. ${ }^{[95]}$ Here, examples of recent approaches to pattern NPs through soft lithography will be reviewed.

\subsubsection{Patterning by contact printing}

\subsubsection{Nanoparticles as inks}

When directly using NPs as ink in $\mu \mathrm{CP}$, the key to successful printing is to tune the surface energy of the particles on the surface. The inking method, the printing conditions, the chemical functions of the particle surface and the substrate surface are all important. The interaction of the NPs with the targeting surface should be stronger than with the stamp surface. 
Soft Lithography for Patterning Self-assemling Systems

The earliest example is from the Whitesides group. ${ }^{[96]}$ Palladium NPs were used as inks first absorbed onto a PDMS surface and then stamped onto amino-functionalized substrates. The NPs were transferred to the substrate due to the affinity for the amino surface. The patterned NPs were then used for the selective electroless deposition of copper.

In order to get better ordering of the NPs patterns, Andres et al. used the LangmuirBlodgett (LB) technique to ink the PDMS surface with NPs. ${ }^{[97]}$ Dense and hexagonally packed monolayers of NPs were assembled first on the air-water interface and transferred to the PDMS stamp surface via an LB setup. Subsequently, the monolayer of densely packed NPs was printed to another substrate. Multilayers of NPs were prepared by repeating the printing process in a layer-by-layer (LBL) scheme, in which subsequent particle layers may be made up of the same or different types of particles. The same method has been applied to print monolayers of magnetic FePt NPs to different substrates to form micrometer-size circles, lines, and squares. ${ }^{[98]}$

In other studies, the PDMS surface is tailored to fine-tune the interactions between the NPs and the stamp surface. Fuchs et al. reported that the distribution of the NPs on a structured stamp surface can be controlled by the gas flow rate during the inking process as well as the type and scale of the patterns on the stamp. CdTe NPs stabilized with thioglycolic acid (TGA) were patterned on $\mathrm{SiO}_{2} / \mathrm{Si}$ surfaces. ${ }^{[99]}$ Bulovic et al. demonstrated a contact printing method for depositing patterned Quantum Dot (QD) monolayer films that are formed by spin-casting QDs onto chemically functionalized PDMS stamps. ${ }^{[100]}$ The chemical functionalization of the PDMS surface was achieved by a coating of a chemical vapor deposited parylene-C layer. Parylene-C is an aromatic polymer; therefore, its surface is optimal for minimizing the surface energy of the QD monolayer, thus facilitates the transfer of the QDs to other substrates. Gigli et al. proposed another $\mu \mathrm{CP}$ approach to pattern QDs, using the SU-8 photoresist as a protective layer for the PDMS. They used this technique to fabricate a multilayer, hybrid, white-light emitting diode. The advantage of using SU-8 instead of parylene-C is the possibility of deposition by using a very easy and low-cost spin-coating process. ${ }^{[101,102]}$

Yang's group first reported combined lift-up and $\mu \mathrm{CP}$ techniques to pattern NPs (Figure 7). ${ }^{[103]}$ In their approach, a PDMS stamp with patterned features was brought into 
contact with a NPs film deposited on a silicon substrate. After the sample was heated and the PDMS stamp was carefully peeled away, a single layer of close-packed particles was transferred to the surface of the PDMS stamp and the corresponding pattern was formed on the colloidal crystal film surface. The NPs inked PDMS stamp was then brought into contact with a thin film of polymer, usually poly(vinyl alcohol) (PVA). After the sample was heated above the $T_{g}$ of PVA, the PDMS stamp was peeled off, and the NP film was transferred onto the substrate. Later, they extended this technique to pattern non-closepacked arrays of NPs based on the solvent-swelling and mechanical deformation properties of PDMS. ${ }^{[104]}$ With this approach the lattice structures of the printed 2D particles arrays can be adjusted.

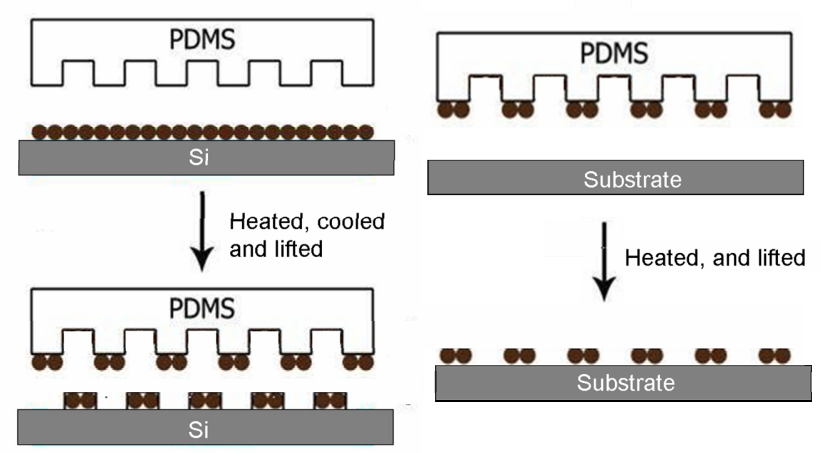

Figure 7. Schematic illustration of lift-up combined with microcontact printing to transfer NPs.

In order to increase the amount of NPs inked onto the stamp surface, our group reported porous stamp structures which are fabricated by one-step phase separation micromolding. With the pore structures functioning as ink reservoirs, multiple printing steps of NPs were achieved without reinking of the stamps. ${ }^{[105]}$

Another method to modify the interactions between NPs and the surface is mixing NPs with other functional materials. Wang et al. reported the contact printing of nanocomposites of a polymer poly(styrene-alt-maleic anhydride), (HSMA) and inorganic NPs (hydrolyzed $\mathrm{TiO}_{2}$ ) from a dispersion of $\mathrm{TiO}_{2} \mathrm{NPs}$ in a HSMA solution. ${ }^{[106]}$ In the last step, calcination of 
Soft Lithography for Patterning Self-assemling Systems

the composites removed the HSMA polymer and resulted in a nanostructure of $\mathrm{TiO}_{2} \mathrm{NPs}$. Bittner et al. reported the $\mu \mathrm{CP}$ of $\mathrm{CdS} /$ dendrimer nanocomposites on a hydroxy-terminated silicon surface. ${ }^{[107]}$ Dendrimers were used as hosts for CdS NPs, and facilitated the adsorption of the NPs to the surface via electrostatic forces, hydrogen bonds and/or Van der Waals interactions.

In our group, we exploited host-guest interactions between dendritic guest molecules and $\beta$-cyclodextrin (CD)-functionalized NPs for the formation of organic/metal NPs multilayers on a PDMS stamp (Figure 8). ${ }^{[108]}$ The multilayer stacks were transferred to a complementary host surface, while no materials remained on the protruding areas of the PDMS stamp. These multilayers showed a well-defined thickness control of $2 \mathrm{~nm}$ per bilayer.
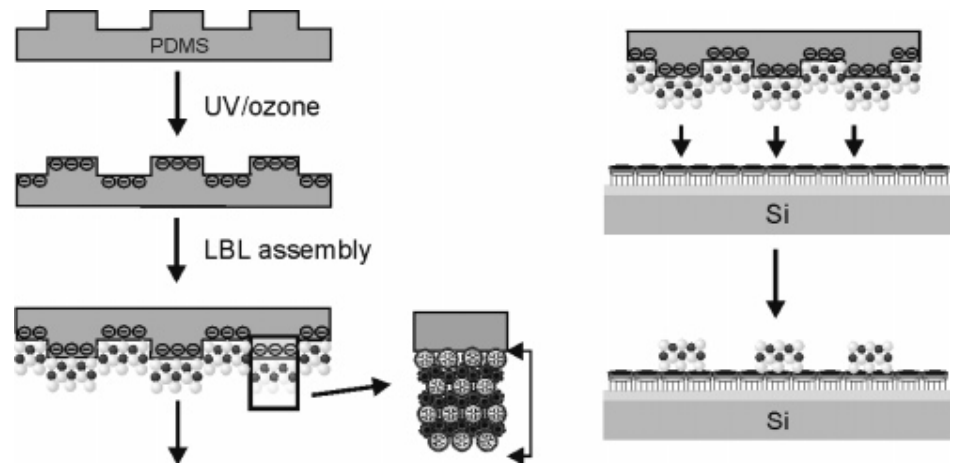

Figure 8. The preparation of a multilayered supramolecular nanostructure on PDMS and transfer printing onto a CD SAM.

\subsubsection{Convective assembly}

The IBM group introduced the concept of convective assembly via an integrated topdown method, called self-assembly, transfer, and integration (SATI) of NPs with high placement accuracy (Figure 9). ${ }^{[109,110]}$ In their method, top-down lithography was used to create a template and to attach and pattern NPs by means of physical confinement. The height of the template was smaller than the size of the NPs in order to have the NPs 
protruding from the pattern. The substrate was treated before hand with a fluoroalkyl SAM to minimize the adhesion between the surface and the NPs. A flat PDMS stamp was used to pick up the patterned NPs to subsequently deposit them onto a Si surface, having a higher adhesion with the NPs than PDMS. By using this method, they have shown the printing of a 60-nm Au NPs array with single-particle resolution. ${ }^{[111]}$

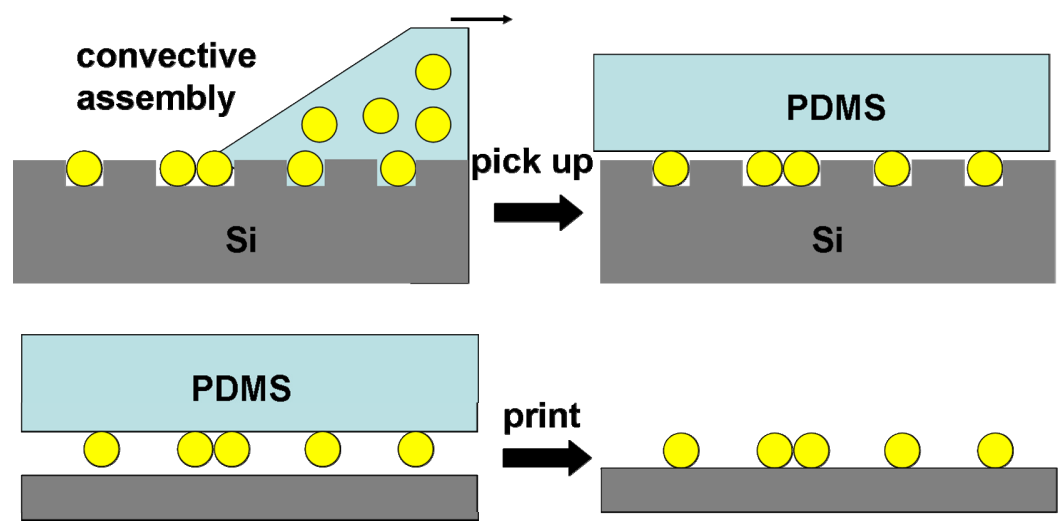

Figure 9. Scheme of convective assembly of NPs, pick-up by a PDMS stamp, and subsequent transfer printing.

In our group, we have combined the concept of convective assembly with supramolecular chemistry to pattern 3D NP structures. $\beta$-Cyclodextrin (CD)-functionalized NPs were first assembled on a patterned PDMS stamp through convective assembly, then adamantyl dendrimers were used as supramolecular glue to chemically bond neighboring NPs together to form stable and ordered 3D hybrid NP structures. These dendrimerinfiltrated NPs could be transfer-printed. ${ }^{[12]}$ Later by using the same method, the 3D hybrid NPs structures were transferred onto topographically patterned substrates via hostguest interactions. Freestanding particle bridges were formed, and the mechanical robustness and rigidity of the particle bridges can be controlled by manipulating the layerby-layer cycles of supramolecular glues of gold nanoparticles and dendrimers (Figure 10). $[112,113]$ 


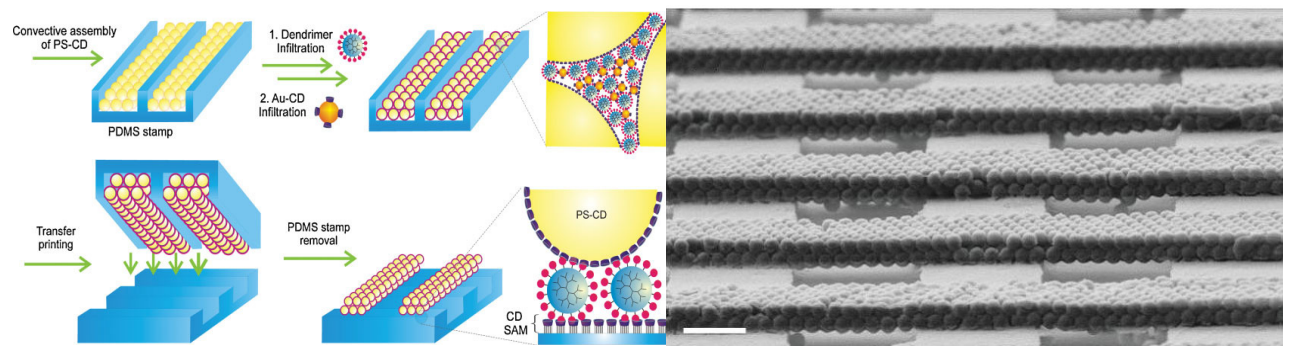

Figure 10. Preparation of hybrid particle bridges on a topographically patterned substrate (left) and SEM image of the free-standing hybrid particle structures (right). Scale bar: 2

$\mu \mathrm{m}$.

\subsubsection{Templated assembly}

In the templated assembly of NPs, organic molecules are first printed onto substrates, and these function as linkers to immobilize NPs via different interactions. Hammond et al. reported the self-organization of $\mathrm{SiO}_{2}$ and polystyrene (PS) NPs on a $\mu \mathrm{CP}$-patterned substrate ${ }^{[114]}$ First, carboxylate-functionalized thiols were printed on a gold surface through $\mu \mathrm{CP}$, then polyelectrolyte multilayers were selectively deposited onto the printing areas. Multicomponent NPs were assembled on the polyelectrolyte surface driven by electrostatic and hydrophobic interactions. The surface charge density was modulated by $\mathrm{pH}$, ionic strength, and effective surface charge of the polyelectrolyte. Kang and Klenerman et al. reported the layer-by-layer ( $\mathrm{LbL}$ ) assembly of quantum dots (QDs) on microcontact printed carboxylate-functionalized SAMs. ${ }^{[15]}$ The LbL assembly was achieved by alternating adsorption of COOH-QDs and 2-mercaptoethanesulfonic acid as the assembly partner. Combinatorially selected peptides and peptide-organic conjugates were used as linkers with controlled structural and organizational conformations to attach QDs at contact printed SAMs. ${ }^{[116]}$ This work establishes a framework for investigating the luminescence properties of surface-immobilized hybrid nanostructures where both the QD-metal distance and the surface attachment density can be monitored and controlled by $\mu \mathrm{CP}$. 


\subsubsection{Patterning by micromolding in capillaries (MIMIC)}

MIMIC is an effective method to pattern or process NPs from their solutions. Whitesides first studied MIMIC to pattern monolayers and multilayers of microspheres. ${ }^{[17]}$ The procedure can fabricate highly ordered 2D and 3D arrays of microspheres by selfassembly. The mechanism of crystallization of latex particles in capillaries involves nucleation due to capillary attractive forces between the microspheres and growth due to evaporation and influx of suspension to compensate for the loss of solvent. Han et al. reported a modified MIMIC approach to fabricate aggregates composed of monodisperse silica microspheres. ${ }^{[118,119]}$ Two different kinds of contact modes, namely, conformal contact and non-conformal contact, between the PDMS mold and the underlying prepatterned substrate, can be controlled during the micromolding, which result in the formation of different aggregates including wood pile, discoid, and rectangular clusters under the influence of template confinement and capillary forces. Recently, monodisperse Si NPs have also been patterned through the MIMIC approach. ${ }^{[120,121]}$ Large area $(\mathrm{cm})$ and ordered NP arrays were successfully fabricated. However, the pattern size is limited by the micron-size mold.

Processing or patterning of nanoparticles (NPs) from their solutions is interesting not only for fundamental research, but also for promising real applications. Blumel et al. reported on the use of MIMIC for patterning silver NPs followed by thermal annealing to fabricate silver source/drain electrodes in well-performing bottom-gate/bottom-contact organic field-effect transistors (OFETs) with poly(3-hexylthiophene) as the active layer material. ${ }^{[122]}$ The transistors they fabricated have performances comparable to corresponding devices based on gold electrodes. Yu et al. applied MIMIC to fabricate stripes of rare-earth ion-doped $\mathrm{LaPO}_{4}$ nanocrystals in a sol-gel process. ${ }^{[123]}$ This class of NPs is particularly important, because they are employed in modern lighting and displays, such as fluorescent lamps, cathode-ray tubes, field-emission displays, and plasma display panels. In their work, they proved the processability of NPs by MIMIC, controlling the pattern morphology, tuning the quantity of material, and the annealing conditions. Recently, Cavallini et al. used MIMIC to pattern magnetic NPs with sub-micrometer periodic features and a vertical resolution of a monolayer. ${ }^{[124]}$ They demonstrated that the morphology of the 
Soft Lithography for Patterning Self-assemling Systems

patterned NPs can be controlled simply by controlling the solution concentration. Exploiting confinement and competing interactions between the adsorbate and the substrate, they fabricated continuous or split stripes composed of $\mathrm{Fe}_{3} \mathrm{O}_{4} \mathrm{NPs}$. Working in a dilute regime, they reached a spatial resolution of a few tens of nanoclusters, depositing a single monolayer of NPs. This approach represents a remarkable example of an integrated topdown/bottom-up process.

\subsubsection{Patterning by soft lithography with solvent mediation}

There are a few other approaches to pattern NPs from their solutions which exploit the self-organization of NPs with the spatial control provided by the PDMS stamp features. Cavallini et al. used a technique they termed 'lithographically controlled wetting' $(\mathrm{LCW}) .{ }^{[125,126]}$ By placing a stamp in contact with a thin liquid film, the capillary forces drive the liquid to distribute only under the protrusions of the stamp. As the solvent evaporates, the deposited solute can form nanostripes on a substrate, and by controlling the concentration and the stamp-substrate distance, the pattern size is controlled. More recently, Cheng et al. have used PDMS stamps to control the shape and location of microdroplets of a solution containing NPs. The pressure exerted on the PDMS stamp and its geometry controls the dewetting dynamics of the solution and allows further control of the local nucleation and growth of superlattices. ${ }^{[127]}$

\subsection{Soft lithography to pattern supramolecular assemblies}

Supramolecular interactions play a pivotal role in biology and are being extensively used for other nonbiological applications as well. ${ }^{[128]}$ Supramolecular interactions are directional, specific, and reversible, which allows fine-tuning of the adsorption/desorption processes at receptors, which is not feasible for conventional routes of immobilization of molecules, assemblies, and particles on surfaces. ${ }^{[129]}$ The combination of soft lithography with supramolecular host-guest interactions has led to the stable positioning and directed assembly of (bio)molecules.

\subsubsection{Affinity contact printing}


The IBM group reported affinity contact printing (Figure 11$){ }^{[130]}$ In their work, the surface of a PDMS stamp was functionalized with anti-mouse $\mathrm{IgG}$ which selectively captured ${ }^{125}$ I-labeled mouse $\mathrm{IgG}$ from a crude biological sample. After rinsing to remove unbound molecules from the stamp surface, the stamp was brought into contact with a solid surface. Because of the stronger interaction between the surface and proteins, the captured molecules were transferred to the solid surface. The same group also demonstrated that protein microarrays can be fabricated by using affinity contact printing. ${ }^{[131]}$ Proteins were selectively picked up from crude biological solutions and then printed on substrates by stamps functionalized with reactive groups that bound the proteins from complex mixtures and aided the transfer of these biomolecules onto the chosen substrates. Recently, Yang et al. reported a method of transferring complementary target DNA from an aqueous solution onto a solid surface by using the concept of affinity microcontact printing. ${ }^{[132]}$

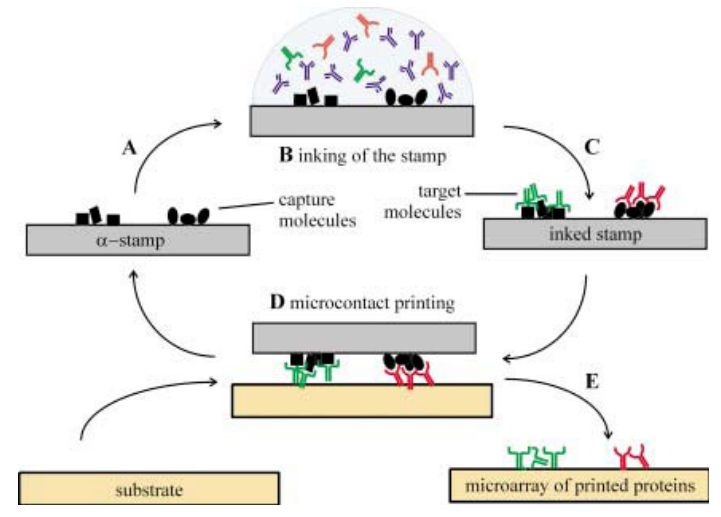

Figure 11. Affinity contact printing.

\subsubsection{Supramolecular nanostamping}

Stellacci's group developed a stamping technique which they called supramolecular nanostamping (SuNS). ${ }^{[133-138]}$ The most interesting of this technique is its ability to print both spatial and chemical information simultaneously. It combines chemical reactions, which contact printing can induce, with DNA molecular recognition. The replication of DNA features is achieved through a hybridization-contact-dehybridization cycle. Features 
Soft Lithography for Patterning Self-assemling Systems

made of different single stranded DNAs can be replicated from a master onto a secondary surface in just three steps. First, the master is immersed in a solution of DNA strands complementary to the ones present on its surface, modified -at their distal position- with a group that can form a chemical bond with another surface (hybridization). Second, another substrate is placed onto the hybridized master (contact). Finally, the two substrates are separated by thermally induced melting of the DNA double helices (dehybridization), achieving a copy of the original pattern. SuNS can reproduce DNA patterns with high resolution $(40 \mathrm{~nm}) \cdot{ }^{[137]}$ Crook' $\mathrm{s}^{[139-142]}$ group reported a similar approach, in which the transfer is based on the affinity between biotin and streptavidin and the separation is obtained through mechanical forces. The limitation here is that DNA molecules need to be first labeled with biotin before they can be transferred.

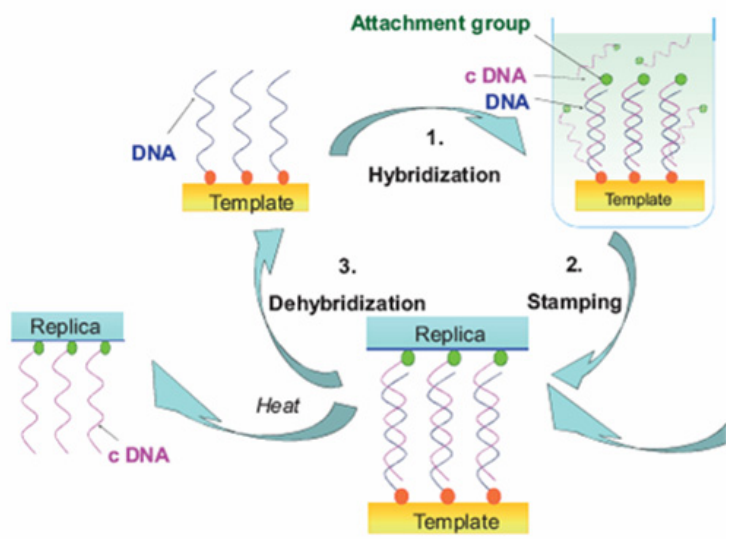

Figure 12. Schematic illustration of the supramolecular nanostamping (SuNS) process.

\subsubsection{Molecular printboards}

Our group has been exploiting supramolecular interactions mainly focusing on multivalency on surfaces. ${ }^{[129]}$ Molecular printboards, ${ }^{[143]}$ which are self-assembled monolayers functionalized with $\beta$-cyclodextrin $(\mathrm{CD})$ as receptor groups, were used as substrates to immobilize molecules through specific and directional supramolecular 
interactions. A typical procedure for fabricating and patterning guest molecules on molecular printboards on gold and silicon oxide surfaces was reported by Auletta et al. ${ }^{[144]}$ A densely packed, well-ordered $\beta$-CD SAM was prepared, and guest-functionalized dendrimers, fluorescently labeled molecules, and polymers were used as inks for supramolecular $\mu \mathrm{CP}$ through host-guest interactions (Figure 13). Comparison with adsorption onto $\mathrm{OH}$-functionalized SAMs showed that the assembly on the printboard was governed by specific, multivalent host-guest interactions. The guest molecules were exclusively found in the areas of preceding contact between the microcontact printing stamp and the substrate, even after extensive rinsing with water or salt solutions. Only rinsing with $10 \mathrm{mM} \mathrm{CD}$, in order to induce competition for binding the adamantyl guest sites, led to noticeable desorption. Very similar results were obtained using adamantylfunctionalized PPI dendrimers. ${ }^{[145]}$

a)

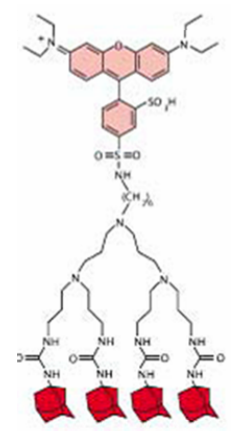

b)

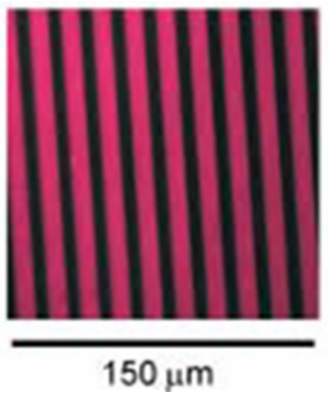

Figure 13. Structure of a lissamine-rhodamine-functionalized dendritic guest molecule (a) and confocal microscopy image after $\mu \mathrm{CP}$ of this guest on a CD-terminated $\mathrm{SAM}$ on $\mathrm{SiO}_{2}$ (b).

Patterning the adamantyl dendrimers on the printboard on silicon oxide provided one of the first cases of the use of two orthogonal interaction motifs for the formation of more complex architectures. ${ }^{[146]}$ The application of a solution of a negatively charged fluorescent dye to a substrate patterned by $\mu \mathrm{CP}$ with the adamantyl dendrimers led to localization of the dyes in the dendrimer-printed areas only. This two-step procedure therefore succumbed to 
Soft Lithography for Patterning Self-assemling Systems

an architecture where the dendrimers were bound by multivalent host-guest interactions, whereas the dyes were immobilized inside the dendrimer cores by electrostatic interactions.

Combining adamantyl dendrimers and $\beta$-CD-functionalized gold particles, various patterning strategies have been developed to create 3D hybrid nanostructures on a printboard by $\mu \mathrm{CP}$ using a layer-by-layer (LBL) approach. ${ }^{[108]}$ By using the nickel(II) complex of a hetero-divalent orthogonal adamantyl nitrilotriacetate linker, hexahistidine (His ${ }_{6}$ )-tagged proteins were successfully patterned on the printboard through $\mu \mathrm{CP}$ in a specific, stable, multivalent manner. ${ }^{[147]}$

Recently, CD-functionalized PDMS stamps were fabricated and successfully used in supramolecular contact printing of specific guest molecules onto printboards. ${ }^{[148]}$ It shows the possibility to selectively recognize ink molecules and to tune the amount of ink molecules transferred. The CD-covered stamps exhibited a highly selective recognition ability when guest-functionalized molecules were captured by self-assembly from ink mixtures. Uniform, equilibrium-controlled host-guest ink transfer was achieved upon conformal contact between two CD-covered surfaces. A supramolecular inkpad has also been developed by using a solid $\beta$-CD printboard, onto which an ink monolayer was specifically assembled, serving as an inkpad. Control over the amount of transferred ink molecules can be exerted by tuning the coverage of the ink monolayer on the inkpad (Figure 14).

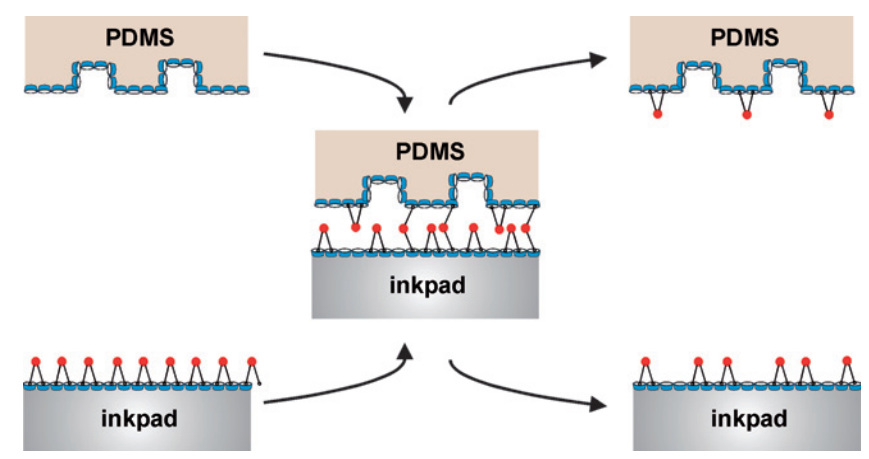

Figure 14. Contact inking with a controlled amount of ink by contacting a CD-modified PDMS stamp with a flat $\beta$-CD printboard on glass (inkpad) with a preadsorbed ink monolayer and subsequent transfer. 


\subsection{Concluding remarks}

Self-assembly of molecular building blocks and nanoparticles by non-covalent interactions is a parallel process that usually occurs in very short times, allowing to create discrete nanostructures and extended 2D and 3D materials. However, free assembly lacks long range order, precision, accuracy, and registration.

With the invention of soft lithography, which brought micro(nano)fabrication within the reach of every chemistry, physics, and biology laboratory, without the need for any significant investment in equipment or cleanroom type infrastructure, researchers have the chance to control the position, order and orientation of self-assembling systems with a large freedom over chemical functionalities which are difficult to be afforded by other lithography techniques. It is the aim of this chapter to provide an overview of recent advances in patterning self-assemble systems by soft lithography. The selected examples focus on molding high resolution features in elastomers that are mechanically stable, the need for specific surface chemistry or template design to adsorb molecules, nanoparticles, and biomolecules from solution onto selected areas of the stamp surface, and new strategies to transfer self-assembly to the target surface. The major efforts were motivated by possible applications in sensing, (bio)analysis, functional nanofabrication, and in fundamental research. Using the possibility of soft lithography in creating chemically distinct patterned SAMs, the elemental basics of chemical, functional nanoparticles, supramolecular, and biological interactions can be studied and understood. The self-assembling behavior in nano-confined conditions is getting more interesting when the patterning resolution is approaching the sub-100 nm range. As such there are plenty of reasons to expect important breakthroughs in the forthcoming years as the field will be deeply investigated.

\subsection{References}

[1] R. Verberg, C. M. Pooley, J. M. Yeomans, A. C. Balazs, Phys. Rev. Lett. 2004, 93. 184501.

[2] M. C. McAlpine, R. S. Friedman, C. M. Lieber, Proceed. IEEE 2005, 93, 1357.

[3] A. Dowling, Nanosciences and nanotechnologies: opportunities and uncertainties, The Royal Society \& The Royal Academy of Engineering, London, July 2004. 
Soft Lithography for Patterning Self-assemling Systems

[4] M. A. Kastner, Phys. Today 1993, 46, 24.

[5] K. K. Likharev, T. Claeson, Sci. Amer. 1992, 266, 80.

[6] M. A. Reed, Sci. Amer. 1993, 268, 118.

[7] V. Vijayakrishnan, A. Chainani, D. D. Sarma, C. N. R. Rao, J. Phys. Chem. 1992, 96, 8679 .

[8] J.-M. Lehn, Angew. Chem. 1990, 102, 1347.

[9] E. Ostuni, L. Yan, G. M. Whitesides, Coll. Surf. B 1999, 15, 3.

[10] A. Ruiz, A. Valsesia, F. Bretagnol, P. Colpo, F. Rossi, Nanotechnology 2007, 18.

[11] I. Bita, J. K. W. Yang, Y. S. Jung, C. A. Ross, E. L. Thomas, K. K. Berggren, Science 2008, 321, 939.

[12] R. A. Segalman, H. Yokoyama, E. J. Kramer, Adv. Mater. 2001, 13, 1152.

[13] J. X. Shi, Z. Wang, P. S. He, H. Y. Yang, Chin. J. Chem. Phys. 2006, 19, 527.

[14] T. I. S. Okazaki, Nature 2000, 1027.

[15] J. Heier, J. Genzer, E. J. Kramer, F. S. Bates, S. Walheim, G. Krausch, J. Chem. Phys. 1999, 111, 11101.

[16] M. Brehmer, L. Conrad, L. Funk, J. Disp. Sci. Tech. 2003, 24, 291.

[17] R. S. Kane, S. Takayama, E. Ostuni, D. E. Ingber, G. M. Whitesides, Biomaterials 1999, 20, 2363.

[18] G. M. Whitesides, E. Ostuni, S. Takayama, X. Y. Jiang, D. E. Ingber, Annu. Rev. Bio. Engin. 2001, 3, 335.

[19] Y. N. Xia, G. M. Whitesides, Ann. Rev. Mater. Sci. 1998, 28, 153.

[20] Y. N. Xia, G. M. Whitesides, Angew. Chem., Int. Ed. Engl. 1998, 37, 551.

[21] Y. N. Xia, J. A. Rogers, K. E. Paul, G. M. Whitesides, Chem. Rev. 1999, 99, 1823.

[22] J. V. Barth, G. Costantini, K. Kern, Nature 2005, 437, 671.

[23] G. M. Whitesides, B. Grzybowski, Science 2002, 295, 2418.

[24] S. J. Holmes, P. H. Mitchell, M. C. Hakey, IBM J. Res. Dev. 1997, 41, 7.

[25] R. H. Stulen, D. W. Sweeney, IEEE J. Quant. Electron. 1999, 35, 694.

[26] J. P. Silverman, J. Vac. Sci. Technol. B 1997, 15, 2117.

[27] M. A. McCord, J. Vac. Sci. Technol. B 1997, 15, 2125.

[28] U. S. Tandon, Vacuum 1992, 43, 241. 
[29] A. Kumar, G. M. Whitesides, Appl. Phys. Lett. 1993, 63, 2002.

[30] Y. N. Xia, E. Kim, G. M. Whitesides, Chem. Mater. 1996, 8, 1558.

[31] E. Kim, Y. N. Xia, G. M. Whitesides, Nature 1995, 376, 581.

[32] Y. N. Xia, G. M. Whitesides, Langmuir 1997, 13, 2059.

[33] X. M. Zhao, A. Stoddart, S. P. Smith, E. Kim, Y. Xia, M. Prentiss, G. M. Whitesides, Adv. Mater. 1996, 8, 420.

[34] E. Kim, Y. N. Xia, X. M. Zhao, G. M. Whitesides, Adv. Mater. 1997, 9, 651.

[35] B. Michel, A. Bernard, A. Bietsch, E. Delamarche, M. Geissler, D. Juncker, H. Kind, J. P. Renault, H. Rothuizen, H. Schmid, P. Schmidt-Winkel, R. Stutz, H. Wolf, IBM J. Res. Dev. 2001, 45, 697.

[36] H. A. Biebuyck, N. B. Larsen, E. Delamarche, B. Michel, IBM J. Res. Dev. 1997, 41,159 .

[37] Y. N. Xia, G. M. Whitesides, J. Am. Chem. Soc. 1995, 117, 3274.

[38] L. Deng, M. Mrksich, G. M. Whitesides, J. Am. Chem. Soc. 1996, 118, 5136.

[39] M. Mrksich, C. S. Chen, Y. N. Xia, L. E. Dike, D. E. Ingber, G. M. Whitesides, Proc. Natl. Acad. Sci. U. S. A. 1996, 93, 10775.

[40] Y. N. Xia, M. Mrksich, E. Kim, G. M. Whitesides, J. Am. Chem. Soc. 1995, 117, 9576.

[41] Q. J. Guo, X. W. Teng, S. Rahman, H. Yang, J. Am. Chem. Soc. 2003, 125, 630.

[42] C. Xu, P. Taylor, M. Ersoz, P. D. I. Fletcher, V. N. Paunov, J. Mater. Chem. 2003, 13,3044 .

[43] S. A. Lange, V. Benes, D. P. Kern, J. K. H. Horber, A. Bernard, Anal. Chem. 2004, $76,1641$.

[44] K. E. Schmalenberg, H. M. Buettner, K. E. Uhrich, Biomaterials 2004, 25, 1851.

[45] J. A. Rogers, K. E. Paul, R. J. Jackman, G. M. Whitesides, J. Vac. Sci. Technol. B 1998, $16,59$.

[46] E. Delamarche, H. Schmid, B. Michel, H. Biebuyck, Adv. Mater. 1997, 9, 741.

[47] F. Bessueille, M. Pla-Roca, C. A. Mills, E. Martinez, J. Samitier, A. Errachid, Langmuir 2005, 21, 12060. 
Soft Lithography for Patterning Self-assemling Systems

[48] I. Bohm, A. Lampert, M. Buck, F. Eisert, M. Grunze, Appl. Surf. Sci. 1999, 141, 237.

[49] K. Glasmastar, J. Gold, A. S. Andersson, D. S. Sutherland, B. Kasemo, Langmuir 2003, 19, 5475 .

[50] J. A. Rogers, R. J. Jackman, G. M. Whitesides, J. L. Wagener, A. M. Vengsarkar, Appl. Phys. Lett. 1997, 70, 7.

[51] D. J. Graham, D. D. Price, B. D. Ratner, Langmuir 2002, 18, 1518.

[52] J. N. Lee, C. Park, G. M. Whitesides, Anal. Chem. 2003, 75, 6544.

[53] A. Ulman, Chem. Rev.1996, 96, 1533.

[54] S. T. Milner, Science 1991, 251, 905.

[55] M. Bal, A. Ursache, M. T. Touminen, J. T. Goldbach, T. P. Russell, Appl. Phys. Lett. 2002, 81, 3479.

[56] G. M. Whitesides, J. P. Mathias, C. T. Seto, Science 1991, 254, 1312.

[57] R. K. Smith, P. A. Lewis, P. S. Weiss, Prog. Surf. Sci. 2004, 75, 1.

[58] N. B. Larsen, H. Biebuyck, E. Delamarche, B. Michel, J. Am. Chem. Soc. 1997, 119, 3017.

[59] E. Delamarche, H. Schmid, A. Bietsch, N. B. Larsen, H. Rothuizen, B. Michel, H. Biebuyck, J. Phys. Chem. B 1998, 102, 3324.

[60] H. Schmid, B. Michel, Macromolecules 2000, 33, 3042.

[61] K. M. Choi, J. Phys. Chem. B 2005, 109, 21525.

[62] K. M. Choi, J. A. Rogers, J. Am. Chem. Soc. 2003, 125, 4060.

[63] K. Y. Suh, R. Langer, J. Lahann, Appl. Phys. Lett. 2003, 83, 4250.

[64] T. Burgin, V. E. Choong, G. Maracas, Langmuir 2000, 16, 5371.

[65] T. W. Odom, J. C. Love, D. B. Wolfe, K. E. Paul, G. M. Whitesides, Langmuir 2002, 18, 5314 .

[66] G. Csucs, T. Kunzler, K. Feldman, F. Robin, N. D. Spencer, Langmuir 2003, 19, 6104

[67] D. Trimbach, K. Feldman, N. D. Spencer, D. J. Broer, C. W. M. Bastiaansen, Langmuir 2003, 19, 10957. 
[68] P. J. Yoo, S. J. Choi, J. H. Kim, D. Suh, S. J. Baek, T. W. Kim, H. H. Lee, Chem. Mater. 2004, 16, 5000.

[69] J. P. Rolland, E. C. Hagberg, G. M. Denison, K. R. Carter, J. M. De Simone, Angew. Chem. Int. Ed. 2004, 43, 5796.

[70] M. J. Hampton, S. S. Williams, Z. Zhou, J. Nunes, D. H. Ko, J. L. Templeton, E. T. Samulski, J. M. DeSimone, Adv. Mater. 2008, 20, 2667.

[71] T. T. Truong, R. S. Lin, S. Jeon, H. H. Lee, J. Maria, A. Gaur, F. Hua, I. Meinel, J. A. Rogers, Langmuir 2007, 23, 2898.

[72] M. Geissler, A. Bernard, A. Bietsch, H. Schmid, B. Michel, E. Delamarche, J. Am. Chem. Soc. 2000, 122, 6303.

[73] E. Delamarche, C. Donzel, F. S. Kamounah, H. Wolf, M. Geissler, R. Stutz, P. Schmidt-Winkel, B. Michel, H. J. Mathieu, K. Schaumburg, Langmuir 2003, 19, 8749 .

[74] S. R. Coyer, A. J. Garcia, E. Delamarche, Angew. Chem. Int. Ed. 2007, 46, 6837.

[75] R. B. A. Sharpe, D. Burdinski, J. Huskens, H. J. W. Zandvliet, D. N. Reinhoudt, B. Poelsema, J. Am. Chem. Soc. 2005, 127, 10344.

[76] Z. Zheng, J. W. Jang, G. Zheng, C. A. Mirkin, Angew. Chem. Int. Ed. 2008, 47, 9951.

[77] R. B. Bass, A. W. Lichtenberger, Appl. Surf. Sci. 2004, 226, 335.

[78] M. Liebau, J. Huskens, D. N. Reinhoudt, Adv. Funct. Mater. 2001, 11, 147.

[79] H. W. Li, D. J. Kang, M. G. Blamire, W. T. S. Huck, Nano Lett. 2002, 2, 347.

[80] S. G. Jang, D. G. Choi, S. Kim, J. H. Jeong, E. S. Lee, S. M. Yang, Langmuir 2006, $22,3326$.

[81] A. Perl, M. Peter, B. J. Ravoo, D. N. Reinhoudt, J. Huskens, Langmuir 2006, 22, 7568 .

[82] T. E. Balmer, H. Schmid, R. Stutz, E. Delamarche, B. Michel, N. D. Spencer, H. Wolf, Langmuir 2005, 21, 622.

[83] Y. L. Loo, R. L. Willett, K. W. Baldwin, J. A. Rogers, J. Am. Chem. Soc. 2002, 124, 7654 
Soft Lithography for Patterning Self-assemling Systems

[84] B. D. Gates, Q. B. Xu, M. Stewart, D. Baldwin, D. Ryan, C. G. Wilson, G. M. Whitesides, Chem. Rev. 2005, 105, 1171

[85] J. A. Helmuth, H. Schmid, R. Stutz, A. Stemmer, H. Wolf, J. Am. Chem. Soc. 2006, $128,9296$.

[86] X. M. Li, M. Peter, J. Huskens, D. N. Reinhoudt, Nano Lett. 2003, 3, 1449.

[87] T. P. Sullivan, M. L. van Poll, P. Y. W. Dankers, W. T. S. Huck, Angew. Chem. Int. Ed. 2004, 43, 4190.

[88] K. S. Park, E. K. Seo, Y. R. Do, K. Kim, M. M. Sung, J. Am. Chem. Soc. 2006, 128,858 .

[89] R. L. C. Alexander A. Shestopalov, J. Toone, J. Am. Chem. Soc. 2007, 129, 13818.

[90] P. W. Snyder, M. S. Johannes, B. N. Vogen, R. L. Clark, E. J. Toone, J. Org. Chem. 2007, 72, 7459.

[91] A. P. Alivisatos, Science 1996, 271, 933.

[92] V. L. Colvin, MRS Bull. 2001, 26, 637.

[93] J. H. Holtz, S. A. Asher, Nature 1997, 389, 829.

[94] I. Gourevich, H. Pham, J. E. N. Jonkman, E. Kumacheva, Chem. Mater. 2004, 16, 1472.

[95] P. A. Maury, D. N. Reinhoudt, J. Huskens, Curr. Opin. Coll. Interf. Sci. 2008, 13, 74.

[96] P. C. Hidber, W. Helbig, E. Kim, G. M. Whitesides, Langmuir 1996, 12, 1375.

[97] V. Santhanam, R. P. Andres, Nano Lett. 2004, 4, 41.

[98] N. Patel, R. Bhandari, K. M. Shakesheff, S. M. Cannizzaro, M. C. Davies, R. Langer, C. J. Roberts, S. J. B. Tendler, P. M. Williams, J. Biomat. Sci.-Polym. E. 2000, 11, 319 .

[99] X. C. Wu, S. Lenhert, L. F. Chi, H. Fuchs, Langmuir 2006, 22, 7807.

[100] L. Kim, P. O. Anikeeva, S. A. Coe-Sullivan, J. S. Steckel, M. G. Bawendi, V. Bulovic, Nano Lett. 2008, 8, 4513.

[101] A. Rizzo, M. Mazzeo, M. Biasiucci, R. Cingolani, G. Gigli, Small 2008, 4, 2143.

[102] A. Rizzo, M. Mazzeo, M. Palumbo, G. Lerario, S. D'Amone, R. Cingolani, G. Gigli, Adv. Mater. 2008, 20, 1886. 
[103] X. Yan, J. M. Yao, G. A. Lu, X. Chen, K. Zhang, B. Yang, J. Am. Chem. Soc. 2004, 126, 10510.

[104] X. Yan, J. M. Yao, G. Lu, X. Li, J. H. Zhang, K. Han, B. Yang, J. Am. Chem. Soc. 2005, 127, 7688 .

[105] H. Xu, X. Y. Ling, J. van Bennekom, X. Duan, M. J. W. Ludden, D. N. Reinhoudt, M. Wessling, R. G. H. Lammertink, J. Huskens, J. Am. Chem. Soc. 2009, 131, 797.

[106] M. T. Wang, H. G. Braun, E. Meyer, Chem. Mater. 2002, 14, 4812.

[107] X. C. Wu, A. M. Bittner, K. Kern, Adv. Mater. 2004, 16, 413.

[108] O. Crespo-Biel, B. Dordi, P. Maury, M. Peter, D. N. Reinhoudt, J. Huskens, Chem. Mater. 2006, 18, 2545.

[109] L. Malaquin, T. Kraus, H. Schmid, E. Delamarche, H. Wolf, Langmuir 2007, 23, 11513.

[110] T. Kraus, L. Malaquin, E. Delamarche, H. Schmid, N. D. Spencer, H. Wolf, $A d v$. Mater. 2005, 17, 2438.

[111] T. Kraus, L. Malaquin, H. Schmid, W. Riess, N. D. Spencer, H. Wolf, Nat. Nanotechnol. 2007, 2, 570.

[112] X. Y. Ling, I. Y. Phang, D. N. Reinhoudt, G. J. Vancso, J. Huskens, ACS Appl. Mater. Interf. 2009, 1, 960.

[113] X. Y. Ling, I. Y. Phang, H. Schönherr, D. N. Reinhoudt, G. J. Vancso, J. Huskens, Small 2009, 5, 1428.

[114] I. S. Lee, P. T. Hammond, M. F. Rubner, Chem. Mater. 2003, 15, 4583.

[115] D. J. Zhou, A. Bruckbauer, C. Abell, D. Klenerman, D. J. Kang, Adv. Mater. 2005, $17,1243$.

[116] M. T. Zin, A. M. Munro, M. Gungormus, N. Y. Wong, H. Ma, C. Tamerler, D. S. Ginger, M. Sarikaya, A. K. Y. Jen, J. Mater. Chem. 2007, 17, 866.

[117] E. Kim, Y. N. Xia, G. M. Whitesides, Adv. Mater. 1996, 8, 245.

[118] W. H. Huang, J. A. Li, L. J. Xue, R. B. Xing, S. F. Luan, C. X. Luo, L. B. Liu, Y. C. Han, Coll. Surf. A 2006, 278, 144.

[119] W. H. Huang, J. Li, C. X. Luo, J. L. Zhang, S. F. Luan, Y. C. Han, Coll. Surf. A 2006, $273,43$. 
Soft Lithography for Patterning Self-assemling Systems

[120] A. Singh, C. K. Malek, S. K. Kulkarni, Smart Mater. Struct. 2008, 17.

[121] Y. Yamauchi, J. Imasu, Y. Kuroda, K. Kuroda, Y. Sakka, J. Mater. Chem. 2009, 19, 1964.

[122] A. Blumel, A. Klug, S. Eder, U. Scherf, E. Moderegger, E. J. W. List, Org. Electron. 2007, 8, 389.

[123] M. Yu, J. Lin, J. Fu, H. J. Zhang, Y. C. Han, J. Mater. Chem. 2003, 13, 1413.

[124] M. Cavallini, E. Bystrenova, M. Timko, M. Koneracka, V. Zavisova, P. Kopcansky, J. Phys.-Condens. Mat. 2008, 20.

[125] M. Cavallini, F. Biscarini, Nano Lett. 2003, 3, 1269.

[126] M. Cavallini, I. Bergenti, S. Milita, G. Ruani, I. Salitros, Z. R. Qu, R. Chandrasekar, M. Ruben, Angew. Chem. Int. Ed. 2008, 47, 8596.

[127] W. L. Cheng, N. Y. Park, M. T. Walter, M. R. Hartman, D. Luo, Nat. Nanotechnol. 2008, 3, 682 .

[128] D. N. Reinhoudt, M. Crego-Calama, Science 2002, 295, 2403.

[129] M. J. W. Ludden, D. N. Reinhoudt, J. Huskens, Chem. Soc. Rev. 2006, 35, 1122.

[130] A. Bernard, D. Fitzli, P. Sonderegger, E. Delamarche, B. Michel, H. R. Bosshard, H. Biebuyck, Nat. Biotechnol. 2001, 19, 866.

[131] J. P. Renault, A. Bernard, D. Juncker, B. Michel, H. R. Bosshard, E. Delamarche, Angew. Chem. Int. Ed. 2002, 41, 2320.

[132] H. Tan, S. Huang, K. L. Yang, Langmuir 2007, 23, 8607.

[133] S. Thevenet, H. Y. Chen, J. Lahann, F. Stellacci, Adv. Mater. 2007, 19, 4333.

[134] A. A. Yu, F. Stellacci, Adv. Mater. 2007, 19, 4338.

[135] O. Akbulut, J. M. Jung, R. D. Bennett, Y. Hu, H. T. Jung, R. E. Cohen, A. M. Mayes, F. Stellacci, Nano Lett. 2007, 7, 3493.

[136] A. A. Yu, F. Stellacci, J. Mater. Chem. 2006, 16, 2868.

[137] A. A. Yu, T. A. Savas, G. S. Taylor, A. Guiseppe-Elie, H. I. Smith, F. Stellacci, Nano Lett. 2005, 5, 1061.

[138] A. A. Yu, T. Savas, S. Cabrini, E. diFabrizio, H. I. Smith, F. Stellacci, J. Am. Chem. Soc. 2005, 127, 16774.

[139] H. H. Lin, L. Sun, R. M. Crooks, J. Am. Chem. Soc. 2005, 127, 11210. 
[140] J. H. Kim, R. M. Crooks, Anal. Chem. 2007, 79, 8994.

[141] J. Kim, R. M. Crooks, Anal. Chem. 2007, 79, 7267.

[142] H. H. Lin, J. Kim, L. Sun, R. M. Crooks, J. Am. Chem. Soc. 2006, 128, 3268.

[143] J. Huskens, M. A. Deij, D. N. Reinhoudt, Angew. Chem. Int. Ed. 2002, 41, 4467.

[144] T. Auletta, B. Dordi, A. Mulder, A. Sartori, S. Onclin, C. M. Bruinink, M. Peter, C. A. Nijhuis, H. Beijleveld, H. Schönherr, G. J. Vancso, A. Casnati, R. Ungaro, B. J. Ravoo, J. Huskens, D. N. Reinhoudt, Angew. Chem. Int. Ed. 2004, 43, 369.

[145] C. M. Bruinink, C. A. Nijhuis, M. Peter, B. Dordi, O. Crespo-Biel, T. Auletta, A. Mulder, H. Schönherr, G. J. Vancso, J. Huskens, D. N. Reinhoudt, Chem. Eur. J. 2005, 11, 3988 .

[146] S. Onclin, J. Huskens, B. J. Ravoo, D. N. Reinhoudt, Small 2005, 1, 852.

[147] M. L. W. Ludden, A. Mulder, K. Schulze, V. Subramaniam, R. Tampe, J. Huskens, Chem. Eur. J. 2008, 14, 2044.

[148] V. B. Sadhu, A. Perl, X. X. Duan, D. N. Reinhoudt, J. Huskens, Soft Matter 2009, 5,1198 . 


\section{Chapter 3}

\section{Bifunctional, Chemically Patterned Flat Stamps for Microcontact Printing of Polar}

\section{Inks*}

$1 \mathrm{H}, 1 \mathrm{H}, 2 \mathrm{H}, 2 \mathrm{H}$-Perfluorodecyltrichlorosilane (PFDTS) and 3-(aminopropyl)triethoxysilane (APTS) were deposited through a mask via two times oxygen plasma onto flat PDMS surfaces to form a bifunctional chemically patterned flat stamp. These stamps were used to transfer polar inks (a thioether-functionalized dendrimer and a fluorescent dye) by microcontact printing. The PFDTS self assembled monolayer (SAM) uses as an effective barrier to prevent ink transfer, while the APTS SAM areas function as an ink reservoir for polar inks. The printing results confirmed excellent transfer of hydrophilic inks with these stamps to gold and glass substrates, even from aqueous solutions. Attachment of a fluorescent dye on the amino-functionalized regions shows the possibility of the further modification of the chemically patterned stamps for tailoring of the stamps' properties.

* Part of this chapter has been published in: X. X. Duan, V. B. Sadhu, A. Perl, M. Peter, D. N. Reinhoudt, J. Huskens, "Bifunctional, Chemically Patterned Flat Stamps for Microcontact Printing of Polar Inks" Langmuir 2008, 24, 3621 


\subsection{Introduction}

In recent years, soft lithography has become a widespread technique to chemically pattern various substrates. ${ }^{[1]}$ As such, microcontact printing $(\mu \mathrm{CP})^{[2,3]}$ has triggered enormous interest because of its processing advantages in comparison to conventional lithographic techniques. In a typical $\mu \mathrm{CP}$ approach, an elastomeric stamp with a relief structure is brought into intimate contact with a substrate to transfer ink molecules from the stamp to the substrate. In the contact areas, the ink forms a self-assembled monolayer (SAM), which can then be used, for instance, as a resist against etching. The lateral dimensions of the SAMs formed depend on the dimensions of the relief features on the stamp. It is possible to form relief features with lateral dimensions as small as $50 \mathrm{~nm}$, but the replication of such structures by $\mu \mathrm{CP}$ remains a challenge. ${ }^{[4-10]}$ The main limiting factors of downsizing the printed patterns are (i) the low mechanical stability of the elastomeric stamp, which is prone to collapse and deformation, ${ }^{[4,11,12]}$ and (ii) the absorption of ink in the stamp promoting ink diffusion and overload of the surface. ${ }^{[13-16]}$ Several solutions have been proposed to overcome these problems such as developing new stamp materials ${ }^{[17-20]}$ and composite stamps ${ }^{[11,21,22]}$ to avoid stamp collapse during printing, inking with ink pads ${ }^{[23]}$ and utilizing heavyweight inks or catalytic microcontact printing to overcome ink diffusion. ${ }^{[8,24,25]}$

Currently, the most common stamp material for $\mu \mathrm{CP}$ is poly(dimethylsiloxane) (PDMS). PDMS is a very soft, chemically cross-linked rubber, which easily establishes conformal contact with a substrate and exhibits excellent printing characteristics with apolar inks, such as alkanethiols. However, the elastomeric character of PDMS is also the origin of some of the most serious technical problems. Deformation of the soft polymer stamps due to non-uniform pressures applied during printing, such as pairing, buckling or roof collapse of structures, renders them unsuitable for high-resolution $\mu \mathrm{CP}$ (see also Chapter 2).

In principle, a flat stamp can solve many or all stamp stability issues. Delamarche et $a l{ }^{[26]}$ have shown that flat PDMS stamps can be patterned by a combination of surface oxidation in an oxygen plasma using a mask and subsequent stabilization of the 
hydrophilized areas by reaction with a poly(ethylene oxide) silane. These stamps have been used for the selective deposition and subsequent patterned transfer of proteins from the stamp surface. In our group, we have introduced the concept of flat, chemically patterned stamps for $\mu \mathrm{CP}$ using regular thiol inks. ${ }^{[27]}$ Stamp functionalization is achieved by local oxidation of a flat piece of PDMS through a mask, followed by adsorption of a fluorinated silane, $1 H, 1 H, 2 H, 2 H$-perfluorodecyltrichlorosilane (PFDTS). It was found that this silane forms densely packed SAMs on oxidized PDMS, which constitutes an effective barrier for ink transfer by $\mu \mathrm{CP}$. These flat PDMS stamps have been used to transfer patterns of alkanethiols to gold. A limitation of these fluorinated silane-modified stamps is that these stamps can not be used to transfer hydrophilic inks, because polar inks do not wet the stamp surface.

In order to solve this problem, a concept of using bifunctional, chemically patterned flat stamps is introduced in this chapter. Besides the fluorinated SAMs, 3-aminopropyltriethoxysilane (APTS) was chosen to form the second SAM type on the remaining PDMS areas. It has short alkyl chains and shows high reactivity in silane chemistry, and the primary amino groups offer the possibility to interact with inks or to further modification to achieve different chemical functionalities on the stamp. APTS has, for example, been used for $\mu \mathrm{CP}$ of DNA via electrostatic interactions. ${ }^{[28]}$ The intermediate hydrophilicity of the APTS layer (typical advancing water contact angle $\theta_{\mathrm{A}}=75^{\circ}$ ) can be advantageous to adsorb polar and apolar inks alike. A bifuntional, flat stamp design is believed to eliminate diffusion (through the strong, specific interaction with the stamp) and overload (since only a monolayer is transferred), therefore leading to improved resolution of the printed patterns.

In this chapter, the formation of the bifunctional, chemically patterned flat PDMS stamps is demonstrated. Three different methods have been used to fabricate flat PDMS stamps functionalized with APTS and PFDTS. XPS, water contact angle, and AFM measurements have been used to identify the two chemical patterns on the PDMS surface. A fluorescent dye was attached to the amino-functionalized regions to investigate the possibilities of the further modification of such bifunctional stamps. These PDMS stamps were used to transfer polar inks to gold and glass substrates. The stability of the chemically patterned PDMS stamps was tested by printing with the same stamps after 1 month. 


\subsection{Results and discussion}

\subsubsection{Stamp fabrication and surface characterization}

\section{Stamp fabrication scheme}

Three different methods have been used to make chemically patterned flat PDMS stamps functionalized with two absorbates (Scheme 1). Since most of the organic solvents swell PDMS, APTS and PFDTS were deposited on the stamp surface by vapor phase deposition. X-ray photoelectron spectroscopy (XPS) and water contact angle (CA) measurements were used to monitor the chemical changes that occur at the stamp surface.

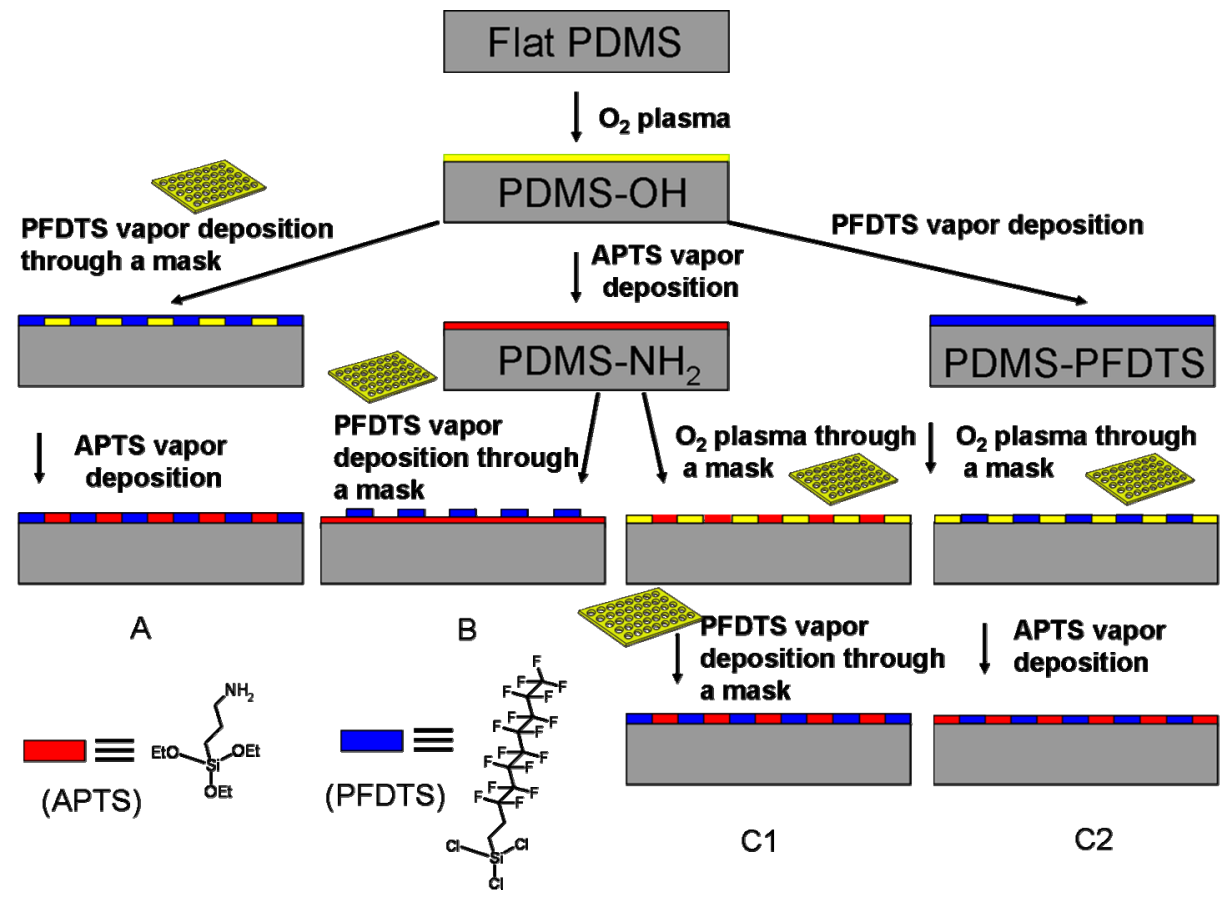

Scheme 1. Fabrication strategies of bifunctional flat PDMS stamps: deposition through a shadow mask (A), grafting on an APTS monolayer (B), two times oxygen plasma with APTS first (C1), and with PFDTS first (C2). 


\section{Deposition through a shadow mask}

In the first method, $1 H, 1 H, 2 H, 2 H$-perfluorodecyltrichlorosilane (PFDTS) and 3aminopropyltriethoxysilane (APTS) were deposited on the PDMS surface using a shadow mask (Scheme 1A). First, a piece of flat PDMS was treated with oxygen plasma. Subsequently, chemical patterns were generated by reaction of the activated PDMS with PFDTS from the vapor phase through a contact mask. In order to prevent the hydrophobic recovery ${ }^{[29]}$ of the oxidized PDMS after the PFDTS deposition the deposition, time was minimized to $15 \mathrm{~min}$ and the annealing step was omitted. After removing the mask, the stamp was immediately transferred into the vapor of APTS for $30 \mathrm{~min}$. So the two different chemical functionalities were patterned on the flat PDMS surface according to the patterns of the contact mask.

Table 1.XPS and water contact angle data of flat PDMS stamp surfaces on full layers of APTS and PFDTTS and on macro-patterned model stamps using different methods shown in Scheme 1.

\begin{tabular}{|c|l|c|c|c|c|}
\hline \multirow{2}{*}{ Samples } & \multicolumn{3}{|c|}{ XPS atomic concentration (\%) } & $\boldsymbol{\theta}_{\mathbf{a}} / \mathbf{\theta}_{\mathbf{r}} \mathbf{~}^{\mathbf{0}}$ ) \\
\cline { 2 - 6 } \multicolumn{2}{|l|}{ APTS (full) } & N1s & F1s & Cl2p & \\
\hline \multicolumn{2}{|c|}{ PFDTS (full) } & 0.49 & 0.11 & 1.76 & $75 / 37$ \\
\hline \multirow{2}{*}{ A } & APTS & 4.95 & 0.21 & 1.73 & $80 / 45$ \\
\cline { 2 - 6 } & PFDTS & 1.94 & 8.56 & 0.36 & $110 / 75$ \\
\hline \multirow{2}{*}{ B } & APTS & 5.48 & 1.28 & 1.55 & $80 / 41$ \\
\cline { 2 - 6 } & PFDTS & 4.05 & 24.26 & 3.69 & $120 / 85$ \\
\hline \multirow{2}{*}{ C1 } & APTS & 6.12 & 0.89 & 1.28 & $75 / 40$ \\
\cline { 2 - 6 } & PFDTS & 0.02 & 21.89 & 0.13 & $118 / 100$ \\
\hline \multirow{2}{*}{ C2 } & APTS & 5.18 & 0.99 & 1.88 & $80 / 42$ \\
\cline { 2 - 6 } & PFDTS & 0.10 & 19.38 & 0.02 & $119 / 102$ \\
\hline
\end{tabular}


In order to facilitate the characterization by contact angle and XPS, a model stamp was prepared according to Scheme 1A, by covering half of a flat piece of PDMS by a piece of Si wafer as the contact mask, thus generating macroscale pattern areas. For comparison, full layers of PFDTS and APTS were deposited on different PDMS slabs. CA measurements were performed to determine the hydrophilicity of the stamp surface. Results are summarized in Table 1.

The full PFDTS layer on PDMS had a typical advancing contact angle $\left(\theta_{\mathrm{A}}\right)$ value of $120^{\circ}$. In contrast, the CA value of the PFDTS layer on the model stamp made by method A was lower $\left(\theta_{\mathrm{a}}=110^{\circ}\right)$. XPS also showed that the fluorine content of the PFDTS layer on the patterned stamp was lower than on the full substrate, and some APTS was deposited within the PFDTS layer as shown by the nitrogen content in the PFDTS layer. These results indicate that the PFDTS did not form a densely packed layer without annealing, and thus resulted in the deposition of APTS everywhere on the stamp surface.

\section{Grafting on an APTS monolayer}

The second method (Scheme 1B) employs the possibilities to attach silanes onto an amino-coated surface. Amino groups of APTS SAMs on silicon react readily with chlorodimethylsilane from the vapor phase ${ }^{[30]}$ Probably the surface-bound amine has a low steric hindrance during this $\mathrm{S}_{\mathrm{N}} 2$ reaction. Here we target the grafting of PFDTS onto a full APTS SAM through a shadow mask. First, a full layer of APTS was deposited on the oxidized flat PDMS surface by vapor phase deposition. Then the chemical patterns were generated by exposure to PFDTS vapor for 20 min through a contact mask. The stamp was annealed at $60{ }^{\circ} \mathrm{C}$ for $3 \mathrm{~h}$ to complete the coupling reaction.

XPS and CA measurements of the macro-patterned model stamp made by this grafting method are summarized in Table 1. Both CA values and XPS data compare well with the full layers of APTS and PFDTS on flat PDMS. However, when using AFM to inspect the SAMs on the stamp surface, the patterns were not well defined (Figure 1). This is attributed to a rather poor quality of the full APTS SAM. This was confirmed by the large hysteresis between the advancing and receding water contact angles $\left(\approx 40^{\circ}\right)$ of this SAM. Apparently, 
this also leads to a poor quality PFDTS SAM on top of the APTS SAM. Different attempts were done to improve the quality of the fluorinated layer, for example, by increase of the annealing time of the bottom APTS layer and extension of the PFDTS grafting reaction time. However, the quality of the patterns did not improve much, according to AFM.
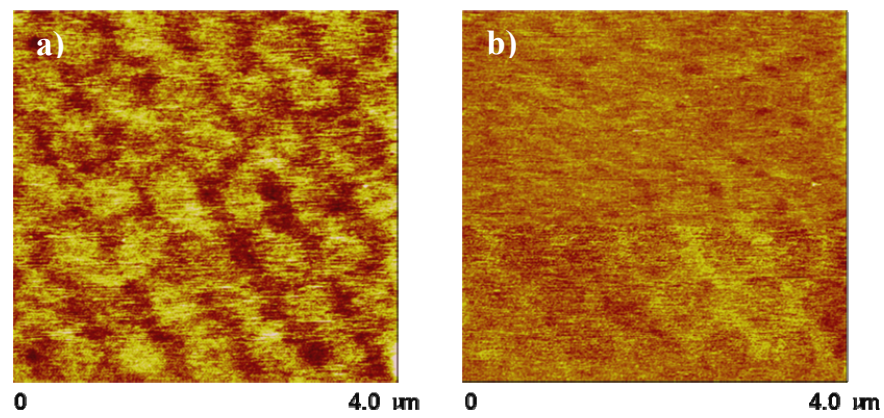

Figure 1. Contact mode AFM (a) height and (b) friction images of the surface of a bifunctional, flat PDMS stamp, which was made by selectively grafting PFDTS onto a full APTS layer (Scheme 1B).

\section{Fabricated through two times oxygen plasma}

As outlined in Scheme 1C, chemically patterned PDMS stamps can also be fabricated via full SAM formation, oxidation through a mask to simultaneously remove the first SAM locally and reactivate the PDMS, followed by attachment of the second silane. Thus, the amino silane can be attached first, followed by the fluorinated silane (Scheme 1C1), or in reverse order (Scheme 1C2). It has been reported that prolonged exposure to oxygen plasma can lead to physical damage of the PDMS surface producing 'cracks' or other microstructures. ${ }^{[31]}$ To our knowledge, no studies have been reported so far on using oxygen plasma twice on PDMS.

In order to make sure the first silane monolayers can be totally removed by $2^{\text {nd }}$ time oxygen plasma and the PDMS surface remain smooth, a carefully study to optimize the $2^{\text {nd }}$ time plasma-etching conditions was performed. The oxidation was done by Plasma Therm 790 reactive ion etching set up with $10 \mathrm{~W}$ power and $10 \mathrm{mTorr} \mathrm{O}_{2}$ pressure. Water contact angle combined with AFM studies were used to check the PDMS surface after different 
time treatment of $2^{\text {nd }}$ time oxygen plasma. First PFDTS was deposited onto a flat PDMS surface and $2^{\text {nd }}$ time oxygen plasma was used to remove the PFDTS SAM. Figure 2 reveals the water contact angle of the PFDTS modified PDMS as a function of the plasma treatment time. As to the result, a PFDTS modified PDMS has a typical water contact angle $\theta=118^{\circ}$. It was found that the oxygen plasma led to a reduction in water contact angle. The lowest value $\left(\theta=16^{\circ}\right)$ was obtained after $20 \mathrm{~s}$ treatment.

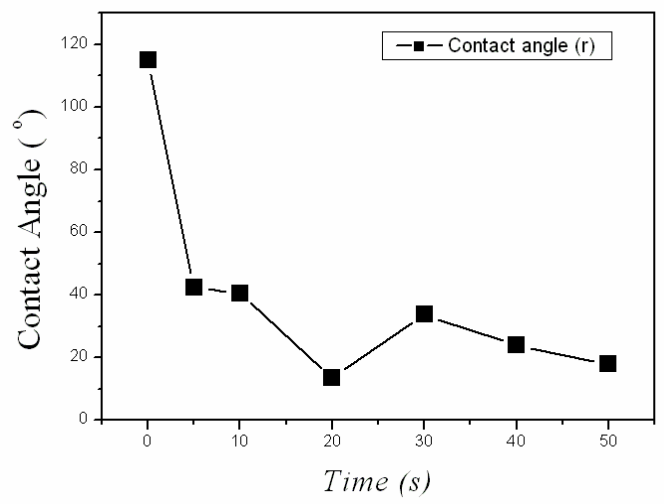

Figure 2. Effect of treatment time in oxygen plasma on the contact angle of water on the surface of PFDTS-modified PDMS.

Figure 3 shows the AFM images of the PFDTS-modified PDMS surface after $2^{\text {nd }}$ time oxygen plasma. It shows that within $20 \mathrm{~s}$ plasma treatment does not lead to visible surface damage. With $30 \mathrm{~s}$ treatment, several small cracks (around $100 \mathrm{~nm}$ ) were observed on the PDMS surface, after $30 \mathrm{~s}$ the cracks become more. This finding also explained the small jump of the water contact angle from 16 to 38 degrees on the PDMS surface after $30 \mathrm{~s}$ oxygen plasma treatment. These experiments confirm the best $2^{\text {nd }}$ time oxygen plasma treatment time is $20 \mathrm{~s}$. 

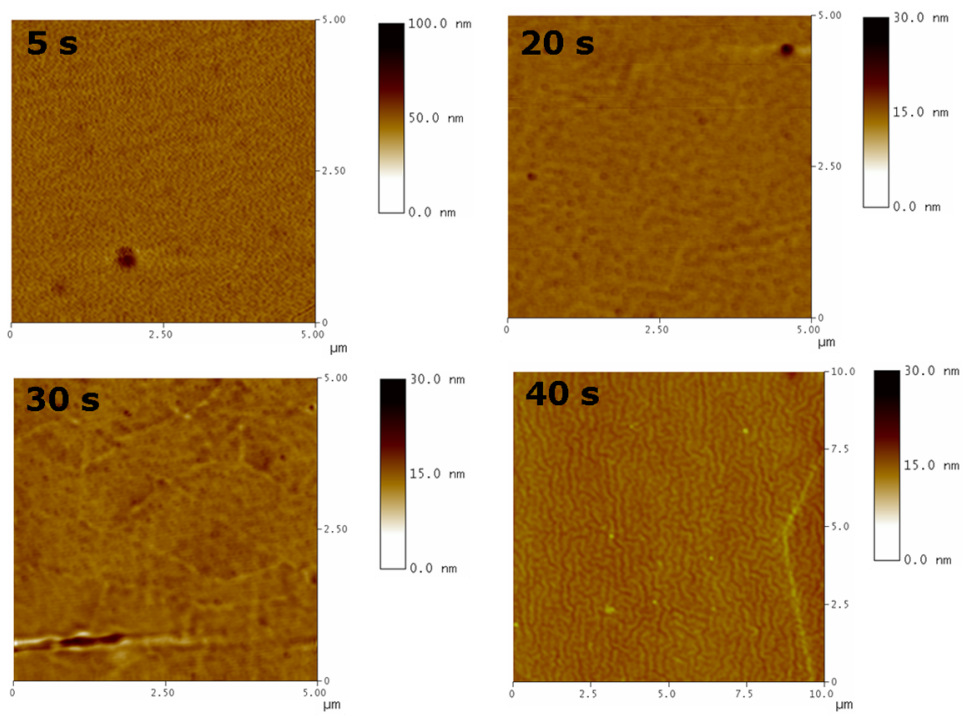

Figure 3. AFM height images of PFDTS-modified PDMS surface after different time oxygen plasma treatment.

XPS and CA measurements of the macro-patterned model stamps made via method $\mathrm{C}$ are summarized in Table 1. Both CA values and XPS results show a clear difference between the APTS- and PFDTS- functionalized regions on the model stamp. The data also compare well with the full layers of APTS and PFDTS on flat PDMS.

Figure 4 shows the AFM height and friction images obtained for a chemically functionalized, submicron patterned stamp made by two times oxygen plasma and deposition of PFDTS first (Scheme 1C2), using the mask with $350 \mathrm{~nm}$ holes. In this case, APTS is expected inside the dot patterns and PFDTS at the surroundings. The height image (Figure 4a) clearly shows well defined $350 \mathrm{~nm}$ dots patterns. A cross-sectional profile (Figure 4c) shows that the dots patterns are $2 \mathrm{~nm}$ high. Since the height difference between a PDFTS and an APTS monolayer is less than $1 \mathrm{~nm}$, the height difference is attributed to the second oxygen plasma treatment. However, such a small height is not expected to influence the contact printing properties. The bright areas in the friction image (Figure $4 \mathrm{~b}$ ) correspond to regions of high friction or strong interaction between the AFM tip and the functional groups on the surface. Combined with the friction loop (Figure 4d), this shows 
that these regions of high friction correspond to the APTS functionalized areas. Low friction regions were at the surroundings, where PFDTS is immobilized. This is in agreement with previous studies, which showed that $\mathrm{Si}_{3} \mathrm{~N}_{4}$ AFM tips have higher frictional forces at surfaces with higher surface energy, here the amino-terminated areas. ${ }^{[32]}$ Moreover, the friction patterns are clearly well defined.

a)

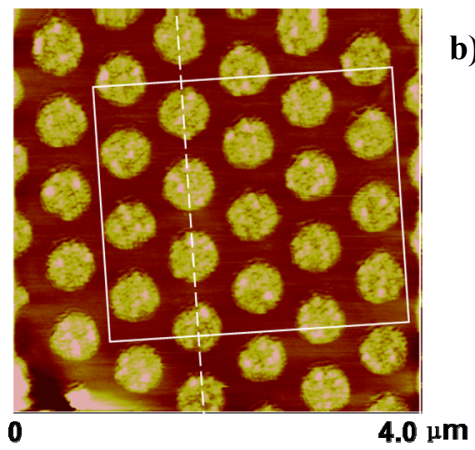

b)

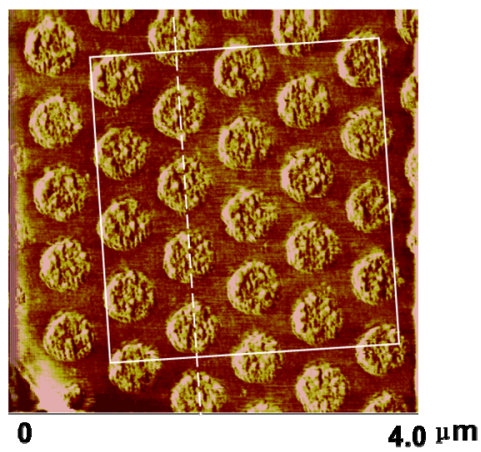

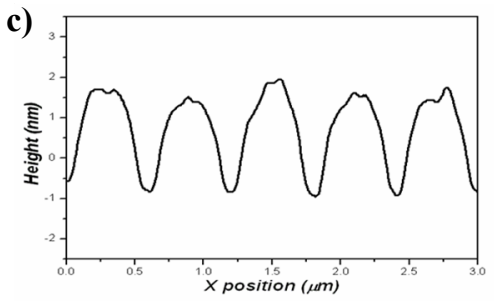

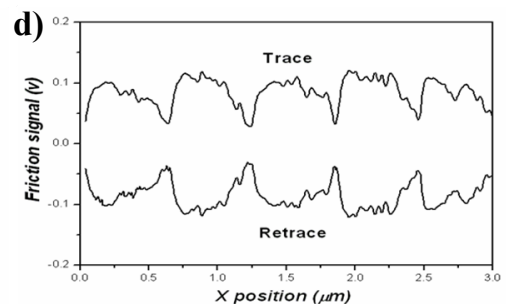

Figure 4. Contact mode AFM height (a) and friction (b) images, and the corresponding

height profile (c), friction loop (d) obtained at an applied normal load of $15 \mathrm{nN}$ of the bifunctional flat PDMS stamp. The stamp was made by two times oxygen plasma in which PFDTS was deposited first (see Scheme 1C2).

In summary, the modification via exposures to oxygen plasma two times is the most efficient method to fabricate bifunctional flat PDMS stamps, and these stamps were therefore used in the remainder of this study. Their stability was shown to be excellent, as printing was reproducible after storing the stamps for a month (see below).

\section{Further modification of amino groups on the stamp surface}


In order to support the formation of amino groups on the stamp surface, these bifunctional stamps were reacted with the fluorescent compound 5-(and-6)carboxytetramethylrhodamine succinimidyl ester (5(6)-TAMRA-SE), of which the succinimidyl ester group reacts readily with amino groups. The attachment of TAMRA was achieved by contact printing using an oxidized flat PDMS stamp, inked with TAMRA-SE, on bifunctional micron-patterned PDMS stamps made by methods C1 and C2 (Scheme 1). To the best of our knowledge, this is the first example that reactive $\mu \mathrm{CP}^{[33-39]}$ is used to functionalize another PDMS surface.
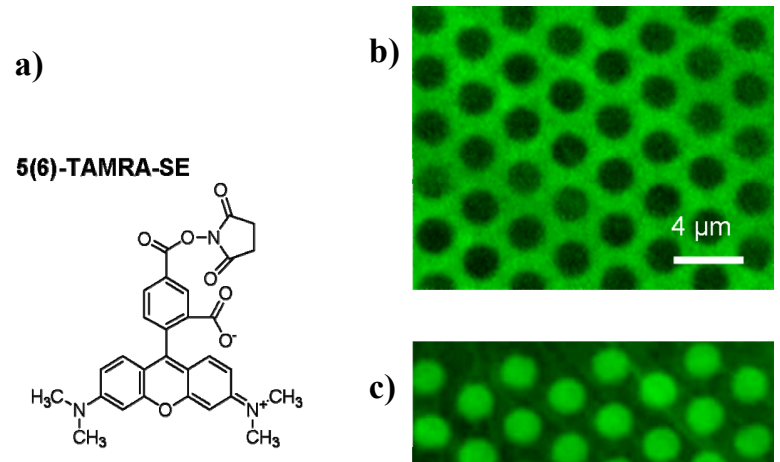

c)

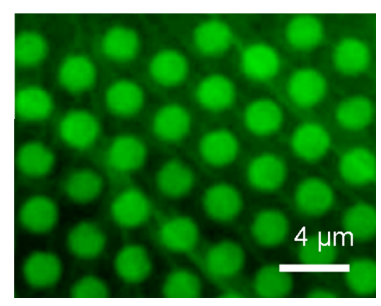

Figure 5. Chemical structure of fluorophore TAMRA-SE (a). Fluorescence microscopy images of the TAMRA patterns on the bifunctional flat PDMS stamps that are fabricated via two exposures to oxygen plasma with method C1 (b) and method C2 (c) (Scheme 1).

Figure 5 shows the fluorescence microscopy images of the PDMS stamps after attachment of TAMRA and excessive rinsing and sonication. All patterns were made using a shadow mask with holes of a diameter of $2 \mu \mathrm{m}$. It is evident from the fluorescence images that the $2 \mu \mathrm{m}$ features of the shadow mask are reproduced well on the PDMS substrate into amino and subsequently fluorescent patterns. The resulting fluorescent TAMRA remained present on the bifunctional PDMS stamps in spite of extensive rinsing and sonication, thus any type of adsorption other than covalent immobilization through amide formation is unlikely. This is also indicated by the fact that TAMRA is absent in the fluorinated areas. 
The successful patterning of fluorescent molecules on a PDMS surface confirms the formation of the two chemically distinct patterns on the PDMS stamp via exposure to oxygen plasma two times and it also shows the possibility of further modification of the amino groups on the PDMS surface to tailor the chemical design of the stamp surface.

\subsubsection{Microcontact printing using bifunctional flat PDMS stamps}

\section{Print G2-S dendrimer through positive printing}

In order to test the stamps in $\mu \mathrm{CP}$, hydrophilic inks were transferred to gold substrates. A second-generation PPI dendrimer (G2-S, Chart 1) with 8 short dialkyl sulfide $\left(\mathrm{CH}_{3}-\mathrm{S}\right.$ $\left.\mathrm{CH}_{2^{-}}\right)$end groups was used as an ink for positive microcontact printing $((+) \mu \mathrm{CP}) .{ }^{[40]} \mathrm{In}$ this technique, the pattern is transferred by printing a poorly etch-resistant ink, followed by immersion of the sample in a solution of a second, etch-resistant adsorbate, which fills the non-contacted areas and acts as a resist in the subsequent etching step.

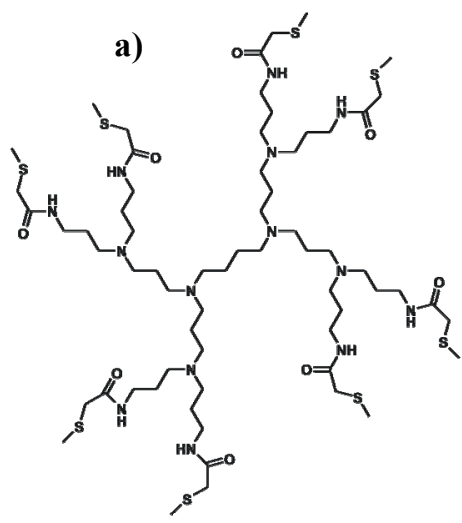

b)<smiles>CN(C)c1ccc(-c2c3ccc(=[N+](C)C)cc-3oc3cc(N(C)C)ccc23)c(C(=O)[O-])c1</smiles>

Chart 1. Chemical structures of polar inks: (a) a second-generation poly(propylene imine) dendrimer with dialkyl sulfide end groups (G2-S), and (b) fluorophore tetramethylrhodamine-5-(and-6)-isothiocyanate (TRITC).

In our group, a series of poly(propylene imine) dendrimers with dialkyl sulfide end groups was prepared and used as inks for $(+) \mu \mathrm{CP}$ on gold. ${ }^{[25]}$ The multivalent sulfide attachment and the relatively high molecular mass of these dendrimers ensured minimal lateral ink spreading and thus optimal feature reproducibility. Among these dendrimeric 
inks, the second-generation dendrimer with short dialkyl sulfide end groups (G2-S) was too polar to be printed by conventional PDMS stamps, and also with oxidized stamps the resolution was poor.

This polar dendritic ink (G2-S, $\mathrm{c} \approx 2 \times 10^{-5} \mathrm{M}$ in ethanol) was printed on a gold surface for 1 min using a bifunctional flat PDMS stamp, prepared according to Scheme 1C2. Thereafter, the samples were immersed in an octadecanethiol (ODT) solution ( $\mathrm{c}=10^{-4} \mathrm{M}$ in ethanol) for $10 \mathrm{~s}$ for back-filling. ODT forms an etch-protective SAM on the rest of the surface. After printing and back-filling, the gold substrates were etched in an acidic solution of $10 \mathrm{mM} \mathrm{Fe}\left(\mathrm{NO}_{3}\right)_{3}, 15 \mathrm{mM}$ thiourea and $1.2 \% \mathrm{HCI}$ at $45{ }^{\circ} \mathrm{C}$ for $2.2 \mathrm{~min} .{ }^{[25]}$ The dendrimer SAM was not etch-resistant and the gold underneath the dendrimer was etched away to give the positive pattern of the original shadow mask which was used in the stamp fabrication. Optical microscopy and scanning electron microscopy (SEM) images of etched gold features on a silicon wafer are shown in Figure 6.

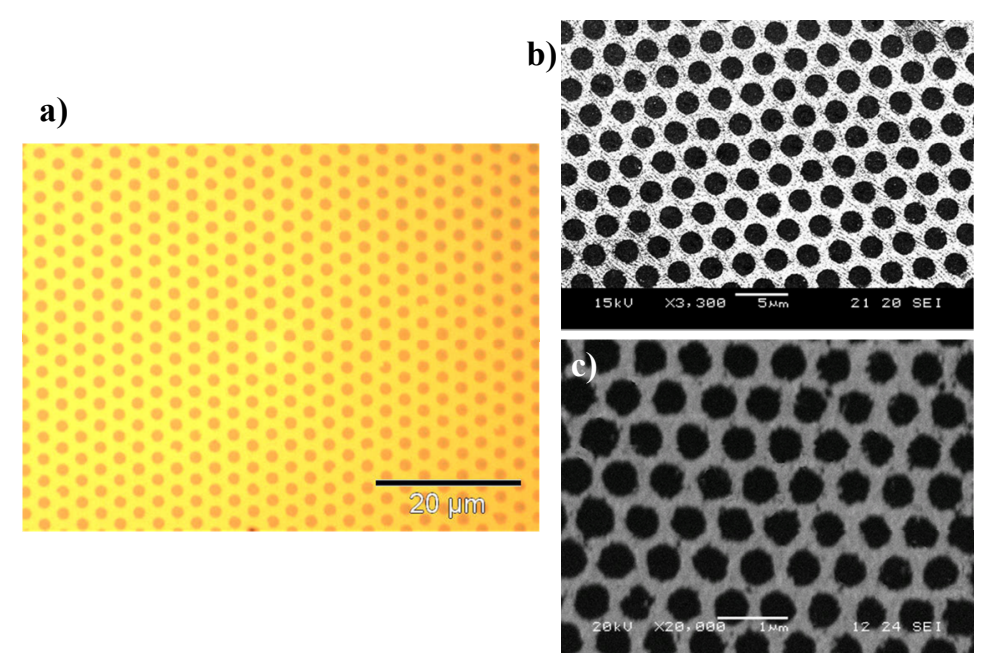

Figure 6. Optical (a) and scanning electron microscopy (SEM) (b) images of $2 \mu \mathrm{m}$ and a SEM image (c) of $350 \mathrm{~nm}$ hole patterns of gold by (+) $\mu C P$ of $G 2 S$ using bifunctional flat PDMS stamps and wet etching. Stamps were fabricated according to Scheme 1C2.

In Figure 6, the dark areas in the images correspond to the printed parts of G2-S, and the bright areas to the back-filled, etch-resistant ODT SAM areas. Figure 6a,b shows the 2 
$\mu \mathrm{m}$ good quality gold patterns over a large area without defects. Using stamps made by a shadow mask with a hole diameter of $350 \mathrm{~nm}$, replication was also achieved (Figure 6c), now leading to features of about $450 \mathrm{~nm}$. The size enlargement is attributed in part to the steps involving the mask in the stamp fabrication process and in part to the wet etching of the final structure after the contact printing process.

\section{Print TRITC dyes}

To show the chemical versatility of the APTS- and PFDTS-modified stamps using a different ink-substrate combination, the hydrophilic and fluorescent tetramethylrhodamine5-(and-6)-isothiocyanate (TRITC) was chosen as an easily traceable ink (Chart 1b). A patterned bifunctional stamp with $4 \mu \mathrm{m}$ dots was made according to Scheme $1 \mathrm{C} 1$. The stamp was inked with an aqueous TRITC solution $(0.1 \mathrm{mM})$. Because of its hydrophilicity, TRITC is expected to reside at the amino-functionalized areas. Thereafter, it was transferred to a clean glass substrate. Figure 7 shows the resulting fluorescence patterns, giving direct evidence of transfer of the ink molecules from the stamp to the substrate. This indicates that aqueous ink solutions can also be used for $\mu \mathrm{CP}$ with the bifunctional chemically patterned flat stamp.
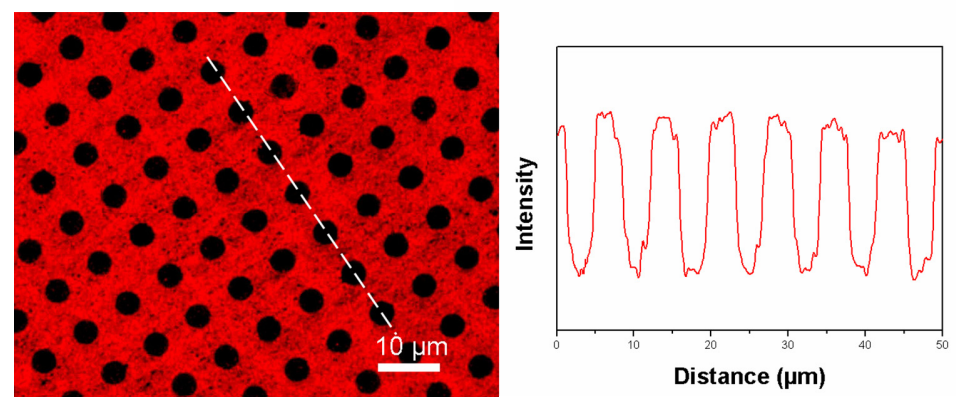

Figure 7. Fluorescence microscopy image after $\mu$ CP of TRITC on a glass substrate. The bifunctional flat PDMS stamp was inked from an aqueous solution $(0.1 \mathrm{mM})$. Printing time was 2 min. Substrates were rinsed with water after printing. The patterned stamps were made from a shadow mask with 4 m holes. 
Bifunctional, Chemically Patterned Flat Stamps for Microcontact Printing of Polar Inks

\section{Stamp stability test}

In order to estimate the stability of the chemically functionalized PDMS stamps, $\mu \mathrm{CP}$ of G2-S was repeated by reusing the same stamps (stored at $-20{ }^{\circ} \mathrm{C}$, under nitrogen), after 1 month. Ink and etching solutions were freshly prepared. After printing and etching, substrates were analyzed by SEM. The resulting gold patterns (Figure 8) were identical to the results of the experiments shown in Figure 3. This indicates a long-term stability of the PFDTS and APTS monolayers on the PDMS surface. This is in contrast to oxidized PDMS, for which hydrophobic recovery occurs after a few hours.

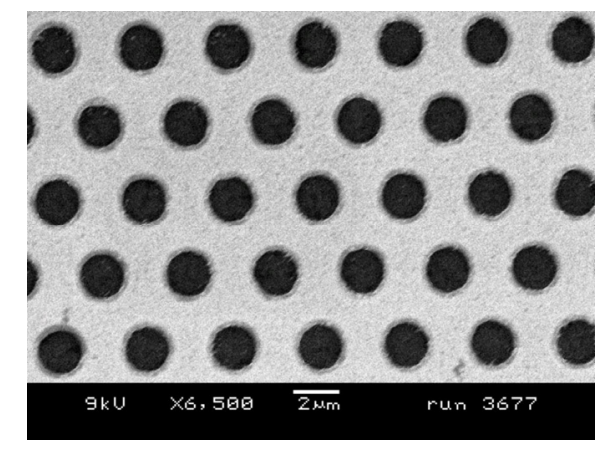

Figure 8. SEM image of $2 \mu \mathrm{m}$ hole patterns of gold patterns printed by bifunctional flat PDMS stamps by (+) $\mu$ CP. Stamps were fabricated according to Scheme $1 C 2$.

\subsection{Conclusions}

Three different methods have been used to fabricate APTS- and PFDTS-functionalized flat PDMS stamps. The method using two times oxygen plasma via full SAM formation, oxidation through a mask to simultaneously remove the first SAM locally and reactivate the PDMS, followed by attachment of the second silane, has been proved to be the most efficient method. XPS, water contact angle, and AFM measurements have confirmed the chemical patterns on the PDMS surface. Attachment of the TAMRA fluorescent dye to the amino-functionalized regions shows the possibility of further modification of the bifunctional stamps to achieve different chemical functionalities on the stamp. This new stamp design showed a good performance in microcontact printing of polar inks (G2-S 
dendrimer and TRITC dye) in both $(+) \mu \mathrm{CP}$ and (-) $\mu \mathrm{CP}$. Printing results of the G2-S dendrimer using the same stamps after one month confirmed the long-term stability of the chemically functionalized PDMS stamps. Future developments will be in the chemical modification of the amine areas for creating specific ink recognition areas.

\subsection{Experimental}

\section{Materials and methods:}

1H,1H,2H,2H-Perfluorodecyltrichlorosilane (PFDTS, purity 97\%), was purchased from ABCR GmbH. 3-(Aminopropyl)triethoxysilane (APTS, purity 99\%) and octadecanethiol (ODT, 98\% purity) were purchased from Sigma-Aldrich. Fluorophores (5(and-6)-carboxytetramethylrhodamine (TAMRA-SE) and tetra-methylrhodamine-5-(and6)-isothiocyanate (TRITC) were obtained from Molecular Probes, Inc. All materials were used as received without further purification. The second generation poly(propylene imine) dendrimer with 8 end groups of dialkyl sulfide (G2-S) was synthesized according to a reported procedure. ${ }^{[25]}$ Microsieves, which consist of a supported metal membrane permeated with micrometer, or submicrometer-sized pores, were used as the masks. Different types of microsieves were purchased from Aquamarijn Micro Filtration B.V., The Netherlands.

\section{Preparation of flat PDMS stamps.}

The stamp material, Sylgard-184 poly(dimethyl siloxane) (PDMS), was obtained from Dow Corning. Flat PDMS stamps were prepared by mixing the curing agent and the prepolymer manually in 1:10 volume ratio and curing overnight at $60{ }^{\circ} \mathrm{C}$ against a polished Si wafer. After curing, the stamps were peeled off from the Si wafer.

\section{Preparation of oxidized PDMS stamps.}

Oxidized flat PDMS stamps were prepared by exposure of flat PDMS stamps to a PlasmaTherm 790 reactive ion etching oxygen plasma $\left(10 \mathrm{~W}, 10 \mathrm{mTorr} \mathrm{O}_{2}\right)$ for $10 \mathrm{~s}$. Oxidized patterns were generated by exposure of the PDMS flat surface to the same oxygen plasma through a microsieve.

\section{Preparation of amino-functionalized PDMS stamps.}


Bifunctional, Chemically Patterned Flat Stamps for Microcontact Printing of Polar Inks

APTS was deposited for $30 \mathrm{~min}$ from its vapor phase onto fully or locally oxidized PDMS stamps, under reduced pressure $\left(\approx 10^{-2}\right.$ to $10^{-3}$ Torr $)$. The adsorbed APTS was assumed to react with the silanol groups present on the oxidized PDMS to yield an aminoterminated stamp. The reaction was carried out in an oven at $90{ }^{\circ} \mathrm{C}$ for $30 \mathrm{~min}$. Physisorbed APTS was removed by sonicating the stamp in ethanol for $3 \mathrm{~min}$.

\section{Preparation of fluorinated PDMS stamps.}

PFDTS was deposited for $30 \mathrm{~min}$ from its vapor phase onto fully or locally oxidized PDMS stamps. The stamps were thereafter cured in an oven for $3 \mathrm{~h}$ at $60{ }^{\circ} \mathrm{C}$.

\section{Attachment of TAMRA onto the bifunctional flat PDMS stamp.}

The attachment of the fluorophore TAMRA to the bifunctional flat PDMS stamp was achieved by putting the bifunctional PDMS stamp in contact with another oxidized flat PDMS stamp which had been inked with $0.23 \mathrm{mM}$ acetonitrile solution of TAMRA-SE for 2.5 min. After separation of the two PDMS slabs, the bifunctional flat PDMS stamp was rinsed with excess ethanol and dried in a nitrogen stream.

\section{G2-S printing.}

Gold substrates were obtained from Ssens BV (Hengelo, The Netherlands) as a layer of $20 \mathrm{~nm}$ gold on titanium $(2 \mathrm{~nm})$ on silicon. Before use, the substrates were treated with oxygen plasma, immersed in ethanol for $1 \mathrm{~h}$, rinsed with water (Millipore) and ethanol, and dried with nitrogen.

The stamps were inked with a few drops of solution of the G2-S in ethanol $\left(10^{-5} \mathrm{M}\right)$ and dried in a stream of nitrogen. After drying the stamps with nitrogen, they were placed manually on the substrate to achieve the conformal contact between the stamp and the substrate. The stamps were pressed slightly against the gold or glass surface at the initial stage of the printing to induce the formation of conformal contact.

An etching solution consisting of $10 \mathrm{mM} \mathrm{Fe}\left(\mathrm{NO}_{3}\right)_{3}, 15 \mathrm{mM}$ thiourea and $1.2 \% \mathrm{HCl}$ was used to etch the dendrimer-printed area on gold in $(+) \mu \mathrm{CP}$. For every experiment the freshly prepared etching solution was kept at $45{ }^{\circ} \mathrm{C}$ in a warm bath for $10 \mathrm{~min}$ before the samples were etched. The etching time was $2.2 \mathrm{~min}$.

\section{TRITC printing.}


TRITC was printed on glass, which was cleaned and activated by submersion in boiling piranha (3:1 mixture of sulfuric acid and hydrogen peroxide. CAUTION! Piranha solutions should be handled with great care in open containers in a fume hood. Piranha is highly corrosive and toxic and potentially explosive.). Stamps were inked by submersion in the solution of TRITC $(0.1 \mathrm{mM})$ in ultrapure water (resistivity $>18.2 \mathrm{M} \Omega \cdot \mathrm{cm}$ ) for $15 \mathrm{~min}$. Prior to printing they were rinsed briefly with ultrapure water and dried in a stream of nitrogen. The glass substrates were contacted with the TRITC- inked stamps for approximately $2 \mathrm{~min}$.

\section{Measurements:}

Contact angles were measured on a Krüss G10 contact angle setup equipped with a CCD camera. Advancing and receding contact angles were determined automatically during growth and reduction of a clean water droplet by the droplet shape analysis routine.

XPS measurements were performed on a Quantera Scanning X-ray Multiprobe instrument from Physical Electronics, equipped with a monochromatic A1 K $\alpha$ X-ray source producing approximately $25 \mathrm{~W}$ of X-ray power. XPS data were collected from a surface area of $1000 \mathrm{pm} \times 300 \mathrm{pm}$ with a pass energy of $224 \mathrm{eV}$ and a step energy of $0.8 \mathrm{eV}$ for survey scans and $0.25 \mathrm{eV}$ for element scans with an equal number of sweep for all elements. Spectra were referenced to the main $\mathrm{C} 1 \mathrm{~s}$ peak set at $284.0 \mathrm{eV}$.

AFM analyses were carried out with a NanoScope Ш (Veeco/Digital Instruments, Santa Barbara, CA, USA) Multimode Atomic Force Microscope equipped with a J-scanner, in contact mode by using $\mathrm{Si}_{3} \mathrm{~N}_{4}$ cantilevers (Nanoprobes, Veeco/Digital Instruments) with a nominal spring constant of about $0.32 \mathrm{Nm}^{-1}$. AFM imaging was performed at ambient conditions.

SEM imaging was carried out with a JEOL, JSM-5610 SEM, operating in secondary electron detection mode.

Fluorescence Microscopy was performed using an Olympus inverted research microscope IX71 equipped with a mercury burner U-RFL-T as light source and a digital camera Olympus DP70 (12.5 million-pixel cooled digital color camera) for image acquisition. Red emission light (A > $590 \mathrm{~nm}$ ) was filtered using a U-MWG Olympus filter cube. 


\subsection{References}

[1] Y. N. Xia, G. M. Whitesides, Angew. Chem. Int. Ed. 1998, 37, 551.

[2] A. Kumar, G. M. Whitesides, Appl. Phys. Lett. 1993, 63, 2002.

[3] Y. N. Xia, G. M. Whitesides, Annu. Rev. Mater. Sci. 1998, 28, 153.

[4] B. Michel, A. Bernard, A. Bietsch, E. Delamarche, M. Geissler, D. Juncker, H. Kind, J. P. Renault, H. Rothuizen, H. Schmid, P. Schmidt-Winkel, R. Stutz, H. Wolf, IBM. J. Res. Dev. 2001, 45, 697.

[5] M. Geissler, H. Wolf, R. Stutz, E. Delamarche, U. W. Grummt, B. Michel, A. Bietsch, Langmuir 2003, 19, 6301.

[6] A. Carvalho, M. Geissler, H. Schmid, B. Michel, E. Delamarche, Langmuir 2002, $18,2406$.

[7] H. W. Li, D. J. Kang, M. G. Blamire, W. T. S. Huck, Nano Lett. 2002, 2, 347.

[8] M. Liebau, J. Huskens, D. N. Reinhoudt, Adv. Func. Mater. 2001, 11, 147.

[9] J. M. McLellan, M. Geissler, Y. N. Xia, J. Am. Chem. Soc. 2004, 126, 10830.

[10] M. Leufgen, A. Lebib, T. Muck, U. Bass, V. Wagner, T. Borzenko, G. Schmidt, J. Geurts, L. W. Molenkamp, Appl. Phys. Lett. 2004, 84, 1582.

[11] A. Bietsch, B. Michel, J. Appl. Phys. 2000, 88, 4310.

[12] K. G. Sharp, G. S. Blackman, N. J. Glassmaker, A. Jagota, C. Y. Hui, Langmuir 2004, 20, 6430 .

[13] E. Delamarche, A. C. F. Hoole, B. Michel, S. Wilkes, M. Despont, M. E. Welland, H. Biebuyck, J. Phys. Chem. B 1997, 101, 9263.

[14] A. P. Quist, E. Pavlovic, S. Oscarsson, Anal. Bio. Chem. 2005, 381, 591.

[15] L. Libioulle, A. Bietsch, H. Schmid, B. Michel, E. Delamarche, Langmuir 1999, 15,300 .

[16] E. Delamarche, H. Schmid, A. Bietsch, N. B. Larsen, H. Rothuizen, B. Michel, H. Biebuyck, J. Phys. Chem. B 1998, 102, 3324.

[17] H. Schmid, B. Michel, Macromolecules 2000, 33, 3042.

[18] D. Trimbach, K. Feldman, N. D. Spencer, D. J. Broer, C. W. M. Bastiaansen, Langmuir 2003, 19, 10957. 
[19] G. Csucs, T. Kunzler, K. Feldman, F. Robin, N. D. Spencer, Langmuir 2003, 19, 6104.

[20] E. Menard, L. Bilhaut, J. Zaumseil, J. A. Rogers, Langmuir 2004, $20,6871$.

[21] M. Tormen, T. Borzenko, B. Steffen, G. Schmidt, L. W. Molenkamp, Microelectron. Eng. 2002, 61-2, 469.

[22] T. W. Odom, J. C. Love, D. B. Wolfe, K. E. Paul, G. M. Whitesides, Langmuir 2002, 18, 5314.

[23] M. Geissler, A. Bernard, A. Bietsch, H. Schmid, B. Michel, E. Delamarche, J. Am. Chem. Soc. 2000, 122, 6303.

[24] X. M. Li, M. Peter, J. Huskens, D. N. Reinhoudt, Nano Lett. 2003, 3, 1449.

[25] A. Perl, M. Peter, B. J. Ravoo, D. N. Reinhoudt, J. Huskens, Langmuir 2006, 22, 7568.

[26] E. Delamarche, C. Donzel, F. S. Kamounah, H. Wolf, M. Geissler, R. Stutz, P. Schmidt-Winkel, B. Michel, H. J. Mathieu, K. Schaumburg, Langmuir 2003, 19, 8749.

[27] R. B. A. Sharpe, D. Burdinski, J. Huskens, H. J. W. Zandvliet, D. N. Reinhoudt, B. Poelsema, J. Am. Chem. Soc. 2005, 127, 10344.

[28] S. A. Lange, V. Benes, D. P. Kern, J. K. H. Horber, A. Bernard, Anal. Chem. 2004, $76,1641$.

[29] D. T. Eddington, J. P. Puccinelli, D. J. Beebe, Sens. Actuators B-Chem. 2006, 114, 170 .

[30] D. G. Kurth, T. Bein, Langmuir 1993, 9, 2965.

[31] M. J. Owen, P. J. Smith, J. Adhes. Sci. Technol. 1994, 8, 1063.

[32] J. L. Wilbur, H. A. Biebuyck, J. C. Macdonald, G. M. Whitesides, Langmuir 1995, 11,825 .

[33] T. Auletta, B. Dordi, A. Mulder, A. Sartori, S. Onclin, C. M. Bruinink, M. Peter, C. A. Nijhuis, H. Beijleveld, H. Schönherr, G. J. Vancso, A. Casnati, R. Ungaro, B. J. Ravoo, J. Huskens, D. N. Reinhoudt, Angew. Chem. Int. Ed. 2004, 43, 369.

[34] J. Lahiri, E. Ostuni, G. M. Whitesides, Langmuir 1999, 15, 2055.

[35] L. Yan, X. M. Zhao, G. M. Whitesides, J. Am. Chem. Soc. 1998, 120, 6179. 
Bifunctional, Chemically Patterned Flat Stamps for Microcontact Printing of Polar Inks

[36] L. Basabe-Desmonts, J. Beld, R. S. Zimmerman, J. Hernando, P. Mela, M. F. G. Parajo, N. F. van Hulst, A. van den Berg, D. N. Reinhoudt, M. Crego-Calama, J. Am. Chem. Soc. 2004, 126, 7293.

[37] T. P. Sullivan, M. L. van Poll, P. Y. W. Dankers, W. T. S. Huck, Angew. Chem. Int. Ed. 2004, 43, 4190.

[38] D. I. Rozkiewicz, B. J. Ravoo, D. N. Reinhoudt, Langmuir 2005, 21, 6337.

[39] D. I. Rozkiewicz, D. Janczewski, W. Verboom, B. J. Ravoo, D. N. Reinhoudt, Angew. Chem. Int. Ed. 2006, 45, 5292.

[40] E. Delamarche, M. Geissler, H. Wolf, B. Michel, J. Am. Chem. Soc. 2002, 124, 3834 . 



\section{Chapter 4}

\section{High-Resolution Contact Printing with}

\section{Chemically Patterned Flat Stamps}

\section{Fabricated by Nanoimprint Lithography*}

Chemically patterned flat stamps provide an effective solution to avoid the mechanical stamp stability problems currently encountered in microcontact printing. A new method was developed to fabricate the chemical patterns on a flat PDMS stamp by using nanoimprint lithography (NIL). The polymer pattern, produced by thermal NIL followed by residual layer removal, acts as a local mask to oxidize the uncovered regions of the PDMS. The chemical patterns were subsequently formed by gas phase evaporation of a fluorinated silane. After removal of the imprint polymer, these stamps were used to transfer alkanethiols as inks to a gold substrate by $\mu C P$. Sub-100 nm gold patterns were successfully replicated by these chemically patterned flat PDMS stamps.

* Part of this chapter has been published in: X. X. Duan, Y. P. Zhao, A. Perl, E. Berenschot, D. N. Reinhoudt, J. Huskens, "High-Resolution Contact Printing with Chemically Patterned Flat Stamps Fabricated by Nanoimprint Lithography" $A d v$. Mater. 2009, 21, 2798. 


\subsection{Introduction}

Microcontact printing $(\mu \mathrm{CP})$ has developed rapidly into a robust printing tool over the past few years because of its processing advantages in comparison to conventional lithographic techniques. ${ }^{[1-4]}$ In a typical $\mu \mathrm{CP}$ scheme, a soft elastomeric stamp is brought into intimate contact with a substrate to transfer ink molecules from the stamp to the substrate. Contact printing is not diffraction limited and can in principle be used to pattern surfaces with sub-100 nm features. However, deformation of the soft polymer stamps due to the limited mechanical stability of the elastomer during stamp fabrication or printing, leading to pairing, buckling or roof collapse of structures, renders them unsuitable for highresolution $\mu \mathrm{CP} .{ }^{[5-8]} \mathrm{New}$ stamp materials ${ }^{[9-13]}$ and composite stamps ${ }^{[6,14,15]}$ may overcome these problems. The mechanical issues are a direct consequence of the inclusion of topographical voids as the transport barriers (see also Chpater 2).

Recently, a new transport barrier concept was introduced by using chemical patterns on a flat stamp surface as the barriers to transfer ink molecules either from the chemically patterned areas (Chapter 3$)^{[16]}$ or from the areas in between. ${ }^{[17,18]}$ In principle, using a chemically patterned flat stamp can solve many or all stamp-stability issues.

A typical way to create chemical patterns on a flat stamp surface is the oxidation of a flat PDMS surface in an oxygen plasma using a shadow mask, followed by stabilization of the hydrophilized areas by reaction with silanes. Functionalization by $1 \mathrm{H}, 1 \mathrm{H}, 2 \mathrm{H}, 2 \mathrm{H}$ perfluorodecyl-trichlorosilane (PFDTS) made it possible to transfer regular thiol inks by such flat stamps. ${ }^{[18]}$ However, the feature size is limited by the shadow mask used. The smallest commercial available shadow mask has $350 \mathrm{~nm}$ features. Another limitation is the difficulty to maintain conformal contact between the mask and the stamp surface during the stamp oxidation step when a sub-500 nm mask is used, thus leading to the loss of resolution.

In this chapter, a new method to prepare chemically patterned flat PDMS stamps by using nanoimprint lithography (NIL) is introduced. Printing of alkanethiols on gold is shown to result in sub-100 nm contact printing.

\subsection{Results and discussion}




\subsubsection{Stamp fabrication scheme}

NIL has been demonstrated as a high-volume and cost-effective patterning technique with sub-10 nm resolution. ${ }^{[19]}$ In this process, a hard mold with nanoscale features is pressed into a thin polymer film which has been coated on a substrate, deforming the shape of the polymer according to the features of the mold and forming a relief pattern in the polymer layer. The patterned polymer layer can be further processed by reactive ion etching, for instance, to transfer the pattern onto the underlying substrate. Here, the NIL-patterned polymer film on a flat PDMS substrate was used as a mask in the oxidation step to fabricate the chemically patterned flat stamp (Scheme 1).

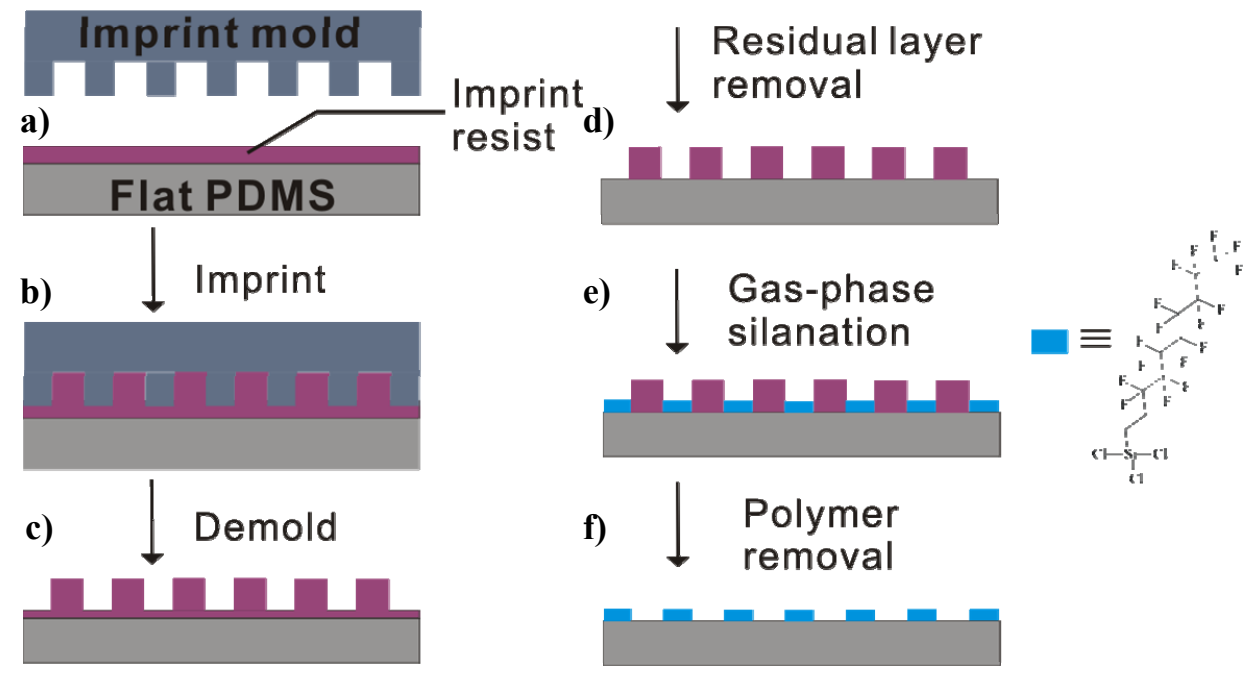

Scheme 1. Schematic illustration of the stamp fabrication: (a) Spin coating of imprint resist mr-I T85 and prebaking, (b) Imprinting the mr-I T85 layer using the NIL mold, (c)

Demolding of the mold from the PDMS substrate, (d) $\mathrm{O}_{2}$ RIE for the "window opening" down to the PDMS substrate and oxidation of the PDMS, (e) Gas-phase silanation of $1 \mathrm{H}, 1 \mathrm{H}, 2 \mathrm{H}, 2 \mathrm{H}$-perfluorodecyl-trichlorosilane (PFDTS) onto the uncovered PDMS; (f) Liftoff of the residual mr-I T85 in cyclohexane.

The polymer pattern, produced by NIL followed by residual layer removal, was used as a local mask to oxidize the flat PDMS substrate on the uncovered regions. Subsequently, the chemical patterns were formed by gas-phase evaporation of the fluorinated silane. In the last step the residual polymer was removed in a solvent. 


\subsubsection{Composite stamps}

Thermal NIL is based on the mechanical molding of a thermoplastic polymer normally on a hard substrate. However, when using PDMS as an imprint substrate, the poor mechanical properties of the PDMS may lead to pattern distortions during the thermal imprint process. Therefore, a composite flat PDMS substrate was fabricated which consisting of commercial PDMS (Sylgard 184) and a $150 \mu \mathrm{m}$ cover glass as a support layer (Figure 1).

a)
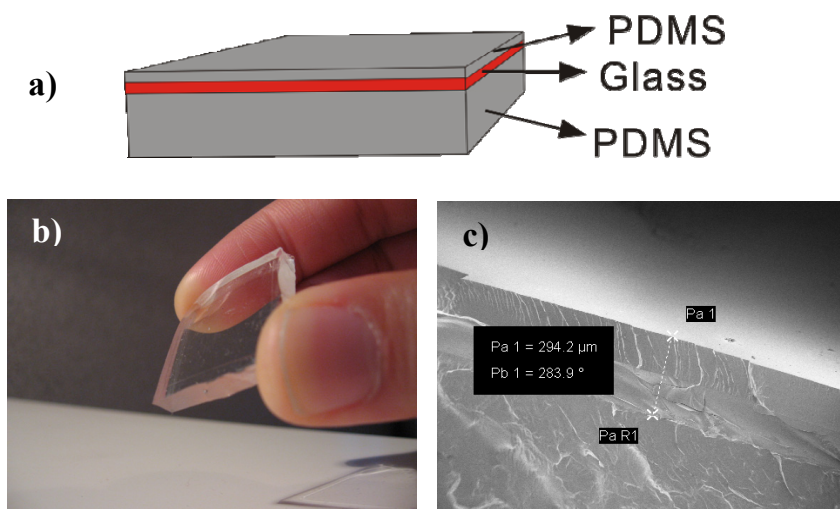

Figure 1. A trilayer composite stamp (a; thin PDMS, cover glass, thick PDMS), example of a composite stamp (b), and SEM cross section image (c) of the composite flat PDMS stamp showing that the thickness of the top PDMS layer is around $300 \mu \mathrm{m}$.

The SEM cross section image shows that the thickness of the top PDMS layer is around $300 \mu \mathrm{m}$ (Figure 1c). The advantage of this method is that the thin glass gives the stamp enough support during imprint, while the composite stamp has enough flexibility to achieve good contact (Figure 1b) in the contact printing step.

\subsubsection{NIL on PDMS}

Another important requirement in the NIL process is the selection of a suitable polymeric resist material. We have used mr-I T85 as the imprint resist, a thermoplastic polymer with a $\mathrm{T}_{\mathrm{g}}$ of $85^{\circ} \mathrm{C}$ which can be imprinted at a relatively low temperature $(130-$ $150^{\circ} \mathrm{C}$ ). Because of the more apolar nature of mr-I T85, the adhesion to the hydrophobic PDMS substrate is better, compared to the more commonly used PMMA. 
Before spin-coating of the imprint resist, the composite PDMS substrate was treated shortly by $\mathrm{O}_{2}$ plasma to achieve a better film quality. The spin-coated film thickness was calculated according to the different pattern sizes and pattern density. Fabrication of NIL patterns with a minimal residual layer is essential to obtain well-defined nanometer-sized patterns, because prolonged exposure to oxygen plasma, which is used in the next step to remove the residual layer, can lead to physical damage of the PDMS surface producing "cracks" or other microstructures. ${ }^{[20]}$ In order to avoid damage to the PDMS surface and to have a more anisotropic etching, the amount of the applied imprint resist was tuned so that the thickness of the residual layer was less than $10 \mathrm{~nm}$.

Initially, two kinds of templates were used, one with a $500 \mathrm{~nm}$ cross bar with a $4 \mu \mathrm{m}$ period (depth $150 \mathrm{~nm}$ ), and another containing holes with half pitch down to $150 \mathrm{~nm}$ (depth $100 \mathrm{~nm})$. Before NIL, the molds were coated by a vapor of the $1 \mathrm{H}, 1 \mathrm{H}, 2 \mathrm{H}, 2 \mathrm{H}$ perfluorodecyl-trichlorosilane (PFDTS) as an anti-adhesion layer for better mold release. ${ }^{[21]}$ Figure 2 shows the imprinted polymer patterns with the two different molds on PDMS substrates. From the AFM cross-section (Figure 2a) it is clear that the polymers have filled all cavities of the mold. In case of dot patterns, replication was also faithful, judging from the feature sizes $(150 \pm 5 \mathrm{~nm})$ which are identical to the original mold features.

An important observation was that, when using PDMS as the imprint substrate, NIL can be performed at very low pressure. A pressure of only 2 bar was sufficient to achieve a good replica during the imprint process. It has been reported before that the imprint pressure can be significantly reduced if a flexible mold is used, which is attributed to conformal contact. ${ }^{[22]}$ The same phenomenon is observed here when using a flexible PDMS substrate. Such a low-pressure process is an advantage to prevent damage to the nanoimprint mold and distortion of the composite PDMS substrate during imprint. 
a)

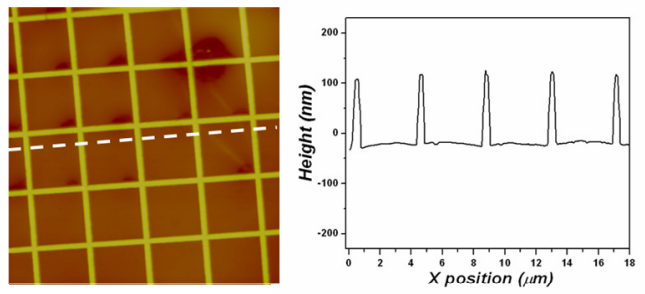

b)

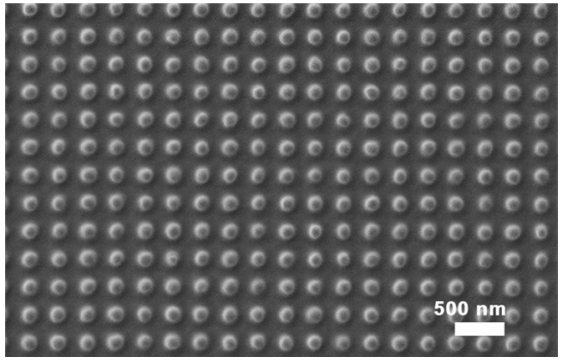

Figure 2. a) AFM height image $(20 \times 20 \mu \mathrm{m})$ and its corresponding cross-sectional profile; b) SEM image of the imprinted (mr-I T85) patterns, on composite PDMS substrates.

\subsubsection{Residual layer removal and pattern transfer}

Residual-layer removal and activation of the exposed areas of the PDMS were achieved in a single $\mathrm{O}_{2}$ reactive-ion-etching (RIE) step. Since the residual polymer layer is approx. $10 \mathrm{~nm}$, the oxygen plasma treatment can be relatively short, thus minimizing damage of the imprinted patterns during etching. Figure 3 shows AFM and SEM images of the patterns after residual- layer removal by $\mathrm{O}_{2}$ RIE. Measured from the AFM height section analyses (Figure $3 \mathrm{a})$, the decrease of the polymer height $(30 \mathrm{~nm})$ compared to the polymer patterns directly after imprint, indicates the successful removal of the residual layer. Measured from the SEM image (Figure 3a), the diameter of the dots patterns are $140 \pm 10 \mathrm{~nm}$ which confirms that RIE did not significantly modify the lateral dimensions of the imprinted features.

Immediately after $\mathrm{O}_{2}$ RIE, PFDTS was deposited from the vapor phase onto the locally oxidized PDMS substrates. The adsorbed PFDTS is assumed to react with the silanol groups present on the oxidized PDMS to yield a PFDTS-modified PDMS stamp. 
Subsequently, the remaining polymer resist was selectively removed by dipping the PDMS substrates into hot cyclohexane $\left(80^{\circ} \mathrm{C}\right)$ for $1 \mathrm{~min}$. It is known that PDMS swells in contact with nonpolar solvents (e.g. hydrocarbons, toluene, and dichloromethane). ${ }^{\left[{ }^{23]}\right.}$ The nonpolar cyclohexane causes some swelling of the PDMS stamp after $1 \mathrm{~min}$, but it was fully reversible upon applying vacuum. The swelling did not influence the chemical patterns on the stamp surface, as proven by the $\mu \mathrm{CP}$ results shown below.

a)
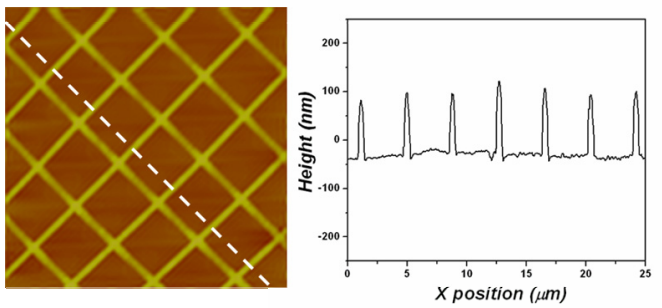

b)

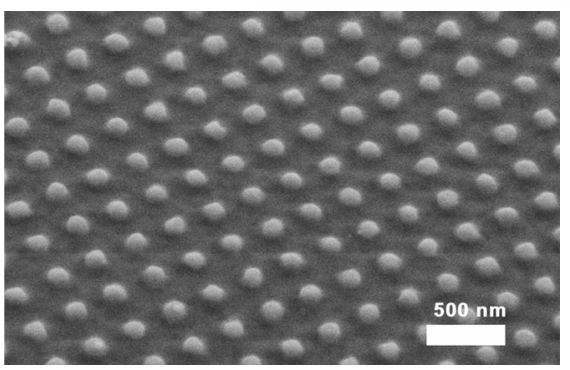

Figure 3. AFM height image $(20 \times 20 \mu \mathrm{m})$ and its corresponding cross-sectional profile $(a)$ and SEM image of the mr-I T85 patterns after $\mathrm{O}_{2} \mathrm{RIE}(b)$.

\subsubsection{Printing results}

The PFDTS-modified flat PDMS stamps were used to transfer patterns of octadecanethiol (ODT) onto gold substrates by $\mu \mathrm{CP}$. After printing, the gold substrates were selectively etched by a ferri-ferrocyanide-based etching solution. ${ }^{[24]}$ Figure 4 shows AFM and SEM images of gold patterns generated by $\mu \mathrm{CP}$ using PFDTS-modified flat PDMS stamps and subsequently wet etching. 
a)

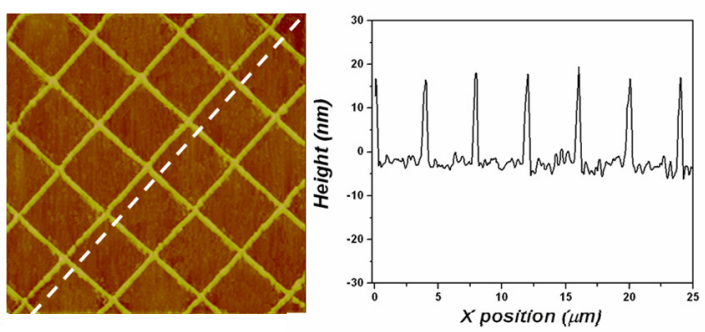

b)

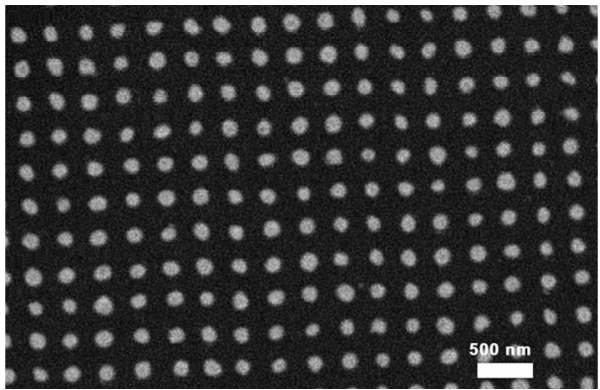

Figure 4. AFM height image $(20 \times 20 \mu \mathrm{m})$ and its corresponding cross-sectional profile $(a)$ and SEM image of the etched gold (b), which were patterned using ODT transferred from PFDTS-modified, patterned flat PDMS stamp (1 mM ODT; printing time: 1 min; etch time: $7 \mathrm{~min})$.

As seen from the AFM height image and height profile (Figure 4a), the gold patterns have a uniform depth of $20 \mathrm{~nm}$. The width of the cross bar lines closely matches the original imprint mold. In case of the dot patterns (Figure 4b), the gold dots have a diameter of $130 \pm 15 \mathrm{~nm}$. The slight decrease (10 nm per side) compared to the original mold is attributed to the isotropic wet etching of the gold. ${ }^{[24]}$

\subsubsection{Sub-100 $\mathrm{nm}$ contact printing}

To test the limitations of the PFDTS-modified flat PDMS stamps, sub-100 nm molds were used to fabricate chemical patterns on the PDMS surface. A mold with nanoridges (50 $\mathrm{nm}$ wide, $100 \mathrm{~nm}$ high, separated by $4 \mu \mathrm{m}$ ) was made by edge lithography followed by wet etching (Figure 5a) ${ }^{[25]}$ Figure 5 b shows the polymer patterns after the imprint on the PDMS substrate. Polymer nanogrooves of $50 \pm 5 \mathrm{~nm}$ separated by $4 \mu \mathrm{m}$ were formed on the PDMS surface. After the polymer patterns were converted into chemical patterns, the PFDTS- 
modified flat PDMS stamps were used in $\mu \mathrm{CP}$. Both regular $(-) \mu \mathrm{CP}$ and positive printing $((+) \mu \mathrm{CP})$ were used to make gold nanopatterns upon wet etching. Upon (-) $\mu \mathrm{CP}$ using ODT, gold nanogrooves were formed with a width of $95 \pm 25 \mathrm{~nm}$ (Figure 5c). The widening is again attributed to the wet-etching step. Upon $(+) \mu \mathrm{CP}$ using a thioether-modified poly(propylene imine) dendrimer, ${ }^{[26]}$ high quality nanolines were formed after gold etching (Figure 5d) with an average size of $85 \pm 10 \mathrm{~nm}$. The slight increase of the width of the gold lines, which is again fully attributable to the wet-etching step, also indicates that ink diffusion is not an issue when printing with such a flat stamp. Thus, in principle, even smaller features can be fabricated when using a high-resolution mold.

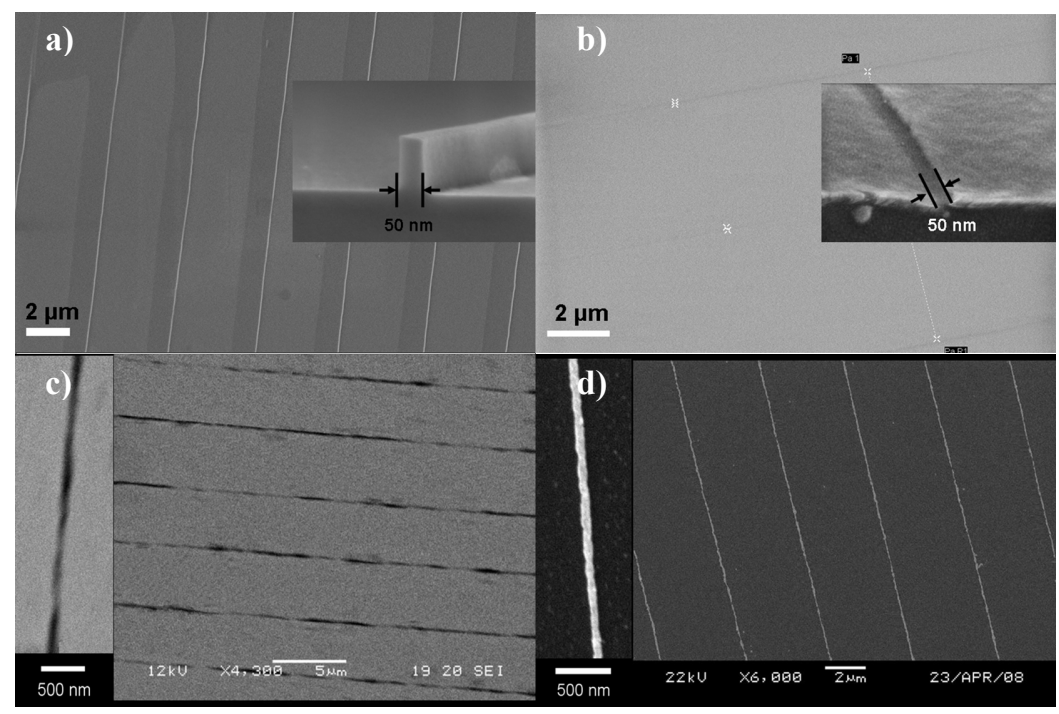

Figure 5. SEM images of sub-100 nm mold (a), the imprinted (mr-I T85) patterns on composite PDMS substrate (b), etched gold, which were patterned using (-) $\mu C P$ (c) and $(+) \mu C P(d)$ using a sub-100 nm PFDTS-modified, patterned flat PDMS stamp.

PFDTS, when attached to oxidized PDMS, is reported to have a surface free energy as low as $12.7 \times 10^{-3} \mathrm{~J} / \mathrm{m}^{2} \cdot{ }^{[27]}$ Therefore PFDTS-modified chemically patterned PDMS stamps are quite stable. ${ }^{[18]}$ We have used the same stamp to print 10 times, over a period of one month. The quality of the etched substrates was identical and independent of the age of the stamp. 


\subsection{Conclusions}

In summary, nanoimprint lithography can be combined with microcontact printing to achieve high-resolution printing. Thermal NIL can be used to fabricate polymer patterns on flat PDMS substrate at low pressure. The polymer pattern can be used as a local mask to oxidize the flat PDMS substrate at the uncovered regions, in order to make flat, chemically patterned stamps. Sub-100 nm gold lines were successfully replicated by these stamps. The resolution is limited only by the mold used in the NIL step, and by the wet-etching step (when metal patterns are targeted). The combined NIL- $\mu \mathrm{CP}$ procedure embodies advantages of NIL (high resolution) with those of $\mu \mathrm{CP}$, both regarding the flexible nature of PDMS (conformal contact in both the NIL and printing steps) and the flat stamp concept (high resolution, elimination of ink diffusion, and stamp deformation). It thus provides a versatile option to create high resolution patterns, especially when combined with large chemical versatility of $\mu \mathrm{CP}$.

\subsection{Experimental}

\section{Materials and methods}

1H,1H,2H,2H-Perfluorodecyltrichlorosilane (PFDTS, purity 97\%) was purchased from ABCR GmbH. Imprint resist mr-I T85 was obtained from Micro Resist. All materials were used as received without further purification. The third generation of thioether-modified poly(propylene imine) dendrimers (G3-M) was synthesized according to a reported procedure. ${ }^{[26]}$ A kit of PDMS prepolymer (Sylgard 184 silicon elastomer) and PDMS curing agent was purchased from Dow Corning Corporation.

\section{Composite flat PDMS substrates}

Composite flat PDMS substrates were prepared by first casting the liquid prepolymer of the elastomer onto a polished Si wafer onto which an anti-adhesion layer had been deposited. After degassing, a piece of cover glass (MENZEL-GLÄSER BB015015A1 15 $\mathrm{mm} \times 15 \mathrm{~mm}$ ) that has been cleaned by a piranha solution (3:1 mixture of sulfuric acid and hydrogen peroxide. CAUTION! Piranha solutions should be handled with great care in open containers in a fume hood. Piranha is highly corrosive and toxic and potentially 
explosive) was then put into the uncured mixture. The cover glass sank to the bottom of the PDMS liquid leaving a thin layer between the cover glass and the Si wafer. After curing at $60{ }^{\circ} \mathrm{C}$ for $8 \mathrm{~h}$ the stamp was peeled off from the Si wafer.

\section{NIL procedure}

Before spin coating the resist, the composite PDMS substrate was treated by short $\mathrm{O}_{2}$ plasma (10 mTorr; $\left.10 \mathrm{~W} ; 20 \mathrm{sccm} \mathrm{O}_{2} ; 15 \mathrm{~s}\right)$. This oxidation step increases the surface energy of the PDMS substrate to achieve a better film quality. A thin film of mr-I T85 solution was spin-coated onto the substrates, followed by soft baking step for 2 min at 140 ${ }^{\circ} \mathrm{C}$ on a hot plate.

The imprint templates were purchased from NIL Technology, one with a $500 \mathrm{~nm}$ cross bar with a $4 \mu \mathrm{m}$ period (depth $150 \mathrm{~nm}$ ), and another containing holes with half pitch down to $150 \mathrm{~nm}$ (depth $100 \mathrm{~nm}$ ). PFDTS was used as an anti-adherent layer to facilitate the stamp-imprint separation.

A stack of a composite PDMS substrate coated with mr-I T85 and a Si template was inserted into a hydraulic press (Specac), and the temperature was raised to $140{ }^{\circ} \mathrm{C}$. Then, 2 bar of pressure was applied to the system for $5 \mathrm{~min}$. Upon cooling to $50{ }^{\circ} \mathrm{C}$, the template was separated from the PDMS substrate.

\section{Pattern transfer}

After imprinting, the residual-layer removal down to the PDMS substrate was achieved via $\mathrm{O}_{2}$ reactive ion etching (RIE) $\left(10 \mathrm{mTorr}, 10 \mathrm{~W}, 20 \mathrm{sccm} \mathrm{O}_{2}\right)$. The etch rate of T85 varied from 1 to $5 \mathrm{~nm} / \mathrm{s}$, depending on the pattern sizes and pattern density. In order to activate the exposed PDMS after residual layer removal, the $\mathrm{O}_{2}$ plasma treatment was extended for another $15 \mathrm{~s}$.

Directly after $\mathrm{O}_{2}$ RIE, PFDTS was immediately deposited for $30 \mathrm{~min}$ from vapor phase onto the locally oxidized PDMS substrates. The adsorbed PFDTS was assumed to react with the silanol groups present on the oxidized PDMS to yield a PFDTS-modified chemically patterned PDMS stamp. The substrates were thereafter cured in an oven for $3 \mathrm{~h}$ at $60{ }^{\circ} \mathrm{C}$ to complete the reaction.

\section{Contact printing}


Gold substrates were obtained from Ssens BV (Hengelo, The Netherlands) as a layer of $20 \mathrm{~nm}$ gold on titanium $(2 \mathrm{~nm})$ on silicon. Before use, the substrates were treated with oxygen plasma, immersed in ethanol for $1 \mathrm{~h}$, rinsed with water (Millipore) and ethanol, and dried with nitrogen.

The stamps were inked with a few drops of ODT solution in ethanol $(1 \mathrm{mM})$ and dried in a stream of nitrogen. After drying the stamps with nitrogen, the stamps were placed manually on the substrate, and were pressed slightly against the gold surface at the initial stage of the printing to induce conformal contact. The printing time was 1 min and the gold samples were selectively etched by keeping the substrate in $\mathrm{S}_{2} \mathrm{O}_{3}{ }^{2-} /$ ferri/ferrocyanide-based etching solutions for different times (in case of features $>100 \mathrm{~nm}, 7 \mathrm{~min}$ etching is used; in the case of sub-100 $\mathrm{nm}$ nano-grooves, 9 min etching is needed to get a clear contrast).

In $(+) \mu \mathrm{CP}$ of the third generation poly(propylene imine) dendrimer with dialkyl sulfide end groups $(\mathrm{G} 3-\mathrm{M})^{[26]}$, the stamp was inked with a solution of G3-M $\left(4 \times 10^{-2} \mathrm{mM}\right)$. Printed was performed for $2 \mathrm{~min}$, and the substrate was subsequently dipped in a $0.1 \mathrm{mM}$ ODT solution for $5 \mathrm{~s}$. A freshly prepared etching solution consisting of $10 \mathrm{mM} \mathrm{Fe}\left(\mathrm{NO}_{3}\right)_{3}, 15 \mathrm{mM}$ thiourea, and $1.2 \% \mathrm{HCl}$ was used to etch the dendrimer-printed areas.

\section{Measurements}

AFM analyses were carried out with a NanoScope W (Veeco/Digital Instruments, Santa Barbara, CA, USA) Multimode Atomic Force Microscope equipped with a J-scanner, in tapping mode by using $\mathrm{Si}_{3} \mathrm{~N}_{4}$ cantilevers (Nanoprobes, Veeco/Digital Instruments) with a nominal spring constant of about $0.32 \mathrm{Nm}^{-1}$. AFM imaging was performed at ambient conditions.

Low-resolution SEM imaging was carried out with a JEOL, JSM-5610 SEM, operating in secondary electron detection mode. High-resolution SEM imaging was carried out with a JEOL Gemini 1550 FEG-SEM.

\subsection{References}

[1] L. Libioulle, A. Bietsch, H. Schmid, B. Michel, E. Delamarche, Langmuir 1999, 15,300 .

[2] A. Kumar, G. M. Whitesides, Appl. Phys. Lett. 1993, 63, 2002. 
[3] Y. N. Xia, G. M. Whitesides, Annu. Rev. Mater. Sci. 1998, 28, 153.

[4] Y. N. Xia, G. M. Whitesides, Langmuir 1997, 13, 2059.

[5] B. Michel, A. Bernard, A. Bietsch, E. Delamarche, M. Geissler, D. Juncker, H. Kind, J. P. Renault, H. Rothuizen, H. Schmid, P. Schmidt-Winkel, R. Stutz, H. Wolf, IBM. J. Res. Dev. 2001, 45, 697.

[6] A. Bietsch, B. Michel, J. Appl. Phys. 2000, 88, 4310.

[7] K. G. Sharp, G. S. Blackman, N. J. Glassmaker, A. Jagota, C. Y. Hui, Langmuir 2004, 20, 6430.

[8] Y. G. Y. Huang, W. X. Zhou, K. J. Hsia, E. Menard, J. U. Park, J. A. Rogers, A. G. Alleyne, Langmuir 2005, 21, 8058.

[9] H. Schmid, B. Michel, Macromolecules 2000, 33, 3042.

[10] D. Trimbach, K. Feldman, N. D. Spencer, D. J. Broer, C. W. M. Bastiaansen, Langmuir 2003, 19, 10957.

[11] G. Csucs, T. Kunzler, K. Feldman, F. Robin, N. D. Spencer, Langmuir 2003, 19, 6104.

[12] E. Menard, L. Bilhaut, J. Zaumseil, J. A. Rogers, Langmuir 2004, 20, 6871.

[13] T. T. Truong, R. S. Lin, S. Jeon, H. H. Lee, J. Maria, A. Gaur, F. Hua, I. Meinel, J. A. Rogers, Langmuir 2007, 23, 2898.

[14] M. Tormen, T. Borzenko, B. Steffen, G. Schmidt, L. W. Molenkamp, Microelectron. Eng. 2002, 61-2, 469.

[15] T. W. Odom, J. C. Love, D. B. Wolfe, K. E. Paul, G. M. Whitesides, Langmuir 2002, 18, 5314 .

[16] X. X. Duan, V. B. Sadhu, A. Perl, M. Peter, D. N. Reinhoudt, J. Huskens, Langmuir 2008, 24, 3621.

[17] E. Delamarche, C. Donzel, F. S. Kamounah, H. Wolf, M. Geissler, R. Stutz, P. Schmidt-Winkel, B. Michel, H. J. Mathieu, K. Schaumburg, Langmuir 2003, 19, 8749.

[18] R. B. A. Sharpe, D. Burdinski, J. Huskens, H. J. W. Zandvliet, D. N. Reinhoudt, B. Poelsema, J. Am. Chem. Soc. 2005, 127, 10344. 
[19] X. Y. Lei, L. Wu, P. Deshpande, Z. N. Yu, W. Wu, H. X. Ge, S. Y. Chou, Nanotechnology 2003, 14, 786.

[20] M. J. Owen, P. J. Smith, J. Adhes. Sci. Technol. 1994, 8, 1063.

[21] G. S. Ferguson, M. K. Chaudhury, H. A. Biebuyck, G. M. Whitesides, Macromolecules 1993, 26, 5870.

[22] D. Y. Khang, H. Kang, T. Kim, H. H. Lee, Nano Lett. 2004, 4, 633.

[23] J. N. Lee, C. Park, G. M. Whitesides, Anal. Chem. 2003, 75, 6544.

[24] Y. N. Xia, X. M. Zhao, E. Kim, G. M. Whitesides, Chem. Mater. 1995, 7, 2332.

[25] Y. P. Zhao, E.; Jansen, H.;Tas, N.; Huskens, J.; Elwenspoek, M., Microelectron. Eng.

[26] A. Perl, M. Peter, B. J. Ravoo, D. N. Reinhoudt, J. Huskens, Langmuir 2006, 22, 7568.

[27] M. K. Chaudhury, G. M. Whitesides, Science 1992, 256, 1539. 


\section{Chapter 5}

\section{Nanopatterning by an Integrated}

\section{Process Combining Capillary Force}

\section{Lithography and Microcontact Printing*}

A novel nanopatterning process was developed by combining capillary force lithography $(C F L)$ and microcontact printing $(\mu C P)$. Flat PDMS was used as the substrate in CFL, and, after chemical functionalization, as the stamp in $\mu C P$, which increased the resolution of both methods. The polymer patterns, produced by CFL on a thin polymer film on the flat PDMS substrate, acted as a mask to oxidize the uncovered regions of the PDMS. The chemical patterns were subsequently formed by gas phase evaporation of a fluorinated silane. After removal of the polymer, these stamps were used to transfer thiol inks to a gold substrate by $\mu C P$. Sub-100 $\mathrm{nm}$ gold patterns were successfully replicated by these chemically patterned flat PDMS stamps.

* Part of this chapter has been published in: X. X. Duan, Y. P. Zhao, A. Perl, E. Berenschot, D. N. Reinhoudt, J. Huskens, "Nanopatterning by an Integrated Process Combining Capillary Force Lithography and Microcontact Printing" Adv. Funct. Mater. 2010, in press 


\subsection{Introduction}

The continued development of lithography technologies allows patterns to be produced with feature sizes well below $100 \mathrm{~nm}$. However, the increasing cost and complexity of these lithographic techniques e.g., deep UV and extreme UV photolithography, ${ }^{[1,2]}$ soft Xray lithography, ${ }^{[3]}$ electron-beam writing, ${ }^{[4]}$ and ion-beam lithography ${ }^{[5]}$ render them less suitable for exploratory research applications. Other ways of patterning structures in the $\sim 10-100 \mathrm{~nm}$ range may represent alternatives if they offer additional advantages in reduced production cost, increased throughput, or more flexibility regarding the material or function of the nanometric structures. The development of soft lithography, ${ }^{[6]}$ which is a family of techniques, is based on the process of molding a soft polymer using hard masters. Varying the way that the molds are used, produces different techniques, the main ones being microcontact printing $(\mu \mathrm{CP}),{ }^{[7]}$ micromolding in capillaries (MIMIC), ${ }^{[8,9]}$ replica molding $(\mathrm{REM}),{ }^{[10]}$ microtransfer molding $(\mu \mathrm{TM}),{ }^{[11]}$ and solvent-assisted micromolding (SAMIM). ${ }^{[12]}$ Among all these techniques microcontact printing $(\mu \mathrm{CP})^{[7,10,13,14]}$ is the most popular one. $\mu \mathrm{CP}$ relies on a reusable template with relief structures predefined by photoor electron-beam lithography from which soft elastomeric stamps (typically polydimethylsiloxane, PDMS) are fabricated. Such a stamp is brought into conformal contact with a substrate, upon which the ink is transferred from the stamp to the substrate surface only at the contact area.

Along with soft lithography, capillary force lithography (CFL) has proven to be quite fruitful. ${ }^{[15,16]}$ It employs capillarity, a useful concept for the patterning of polymer materials and combines the essential feature of nanoimprint lithography (NIL) ${ }^{[17]}$-molding a polymer melt with the use of an elastomeric stamp. The use of a soft stamp provides conformal contact and therefore closure of channels upon contact and concomitant capillary forces, thus allowing the polymer flow in the absence of external pressures. When the polymer film is thick enough, polymer melt can fill up the void space between the polymer and the mold completely, thereby generating a negative replica of the mold. Anisotropic dewetting occurs when the polymer film is relatively thin with respect to the intrusion depth of the stamp, 
Integration of Capillary Force Lithography and Microcontact Printing

thus the CFL process can be tailored to make the desired substrate surface exposed, eliminating the need to open the windows with reactive ion etching (RIE). ${ }^{[15]}$

Both in $\mu \mathrm{CP}$ and CFL, the excellent elastomeric property of PDMS is a key factor. It allows the PDMS mold to intimately contact another substrate during pattern transfer. However, the elastomeric character of PDMS is also the origin of some of the most serious technical problems. Deformation of the soft polymer stamps, such as pairing, buckling or roof collapse of structures, impose a resolution limit both for $\mu \mathrm{CP}$ and CFL. ${ }^{[18-21]}$ Sub-100 $\mathrm{nm}$ pattering is rather difficult by conventional $\mu \mathrm{CP}$ or CFL when a soft stamp is used.

In this chapter, a novel and high throughput method for large area nanopatterning is introduced by combining CFL and $\mu \mathrm{CP}$. In this method, a flat PDMS was used as the substrate in CFL, thus high-resolution (from $500 \mathrm{~nm}$ to sub-100 nm) polymer patterns can be fabricated through CFL on a flat PDMS substrate by directly using a patterned hard $\mathrm{Si}$ wafer as the mold. The polymer patterns were functioned as a mask in a subsequent PDMS oxidation step. Thereafter, chemical patterns were formed by gas phase evaporation of a fluorinated silane. After polymer removal, these chemically patterned flat stamps can be used to transfer alkanethiols as inks to a gold substrate by $\mu \mathrm{CP}$.

The key step in this patterning method is to use flat PDMS both as a substrate in the CFL step, which allows the direct use of a high resolution Si mold, because the PDMS substrate ensures conformal contact with the hard mold and as a flat stamp in $\mu \mathrm{CP}$ to transfer thiol inks to other substrates which has the advantage of high-resolution printing, because of elimination of ink diffusion and stamp deformation. ${ }^{[22-26]}$

\subsection{Results and discussion}

\subsubsection{Overall patterning strategy}

Scheme 1 depicts this nanopatterning approach by combining CFL and $\mu$ CP. As studied by Yoon et. al., CFL is possible with an impermeable hard mold only if the substrate is flexible. ${ }^{[27]}$ In our case, a patterned Si wafer is directly used as the mold and flat PDMS is used as the substrate, which ensures conformal contact between the mold and the substrate, which is essential for capillary rise. However, high-temperature processing is known to 
limit the performance of PDMS. ${ }^{[18]}$ PDMS, the thermoplastic resist and the Si template have different thermal expansion coefficients $\left(\alpha_{\text {PDMS }} \gg \alpha_{\mathrm{Si}}\right)$. It may cause separation of the $\mathrm{Si}$ mold from the PDMS substrate during the CFL process or deformation of the polymer patterns upon cooling. Therefore, a composite flat PDMS substrate was used by curing a thin glass together with the prepolymer of PDMS. The fabrication of the composite flat PDMS has been described in Chapter 4.
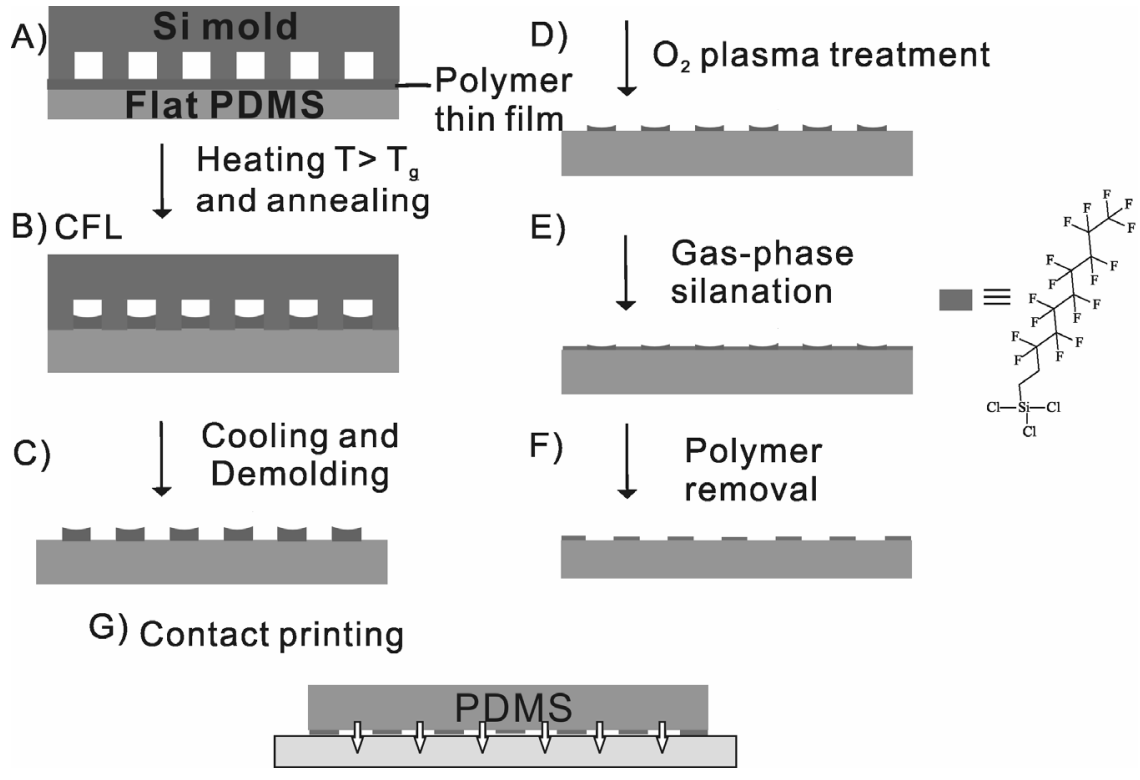

Scheme 1. Schematic illustration of the nanopatterning approach by combining CFL and $\mu C P$ : a) Spin coating of polymer resist mr-I T85 and prebaking, b) CFL is carried out on a thin mr-I T85 layer by using the Si mold, c) Cooling to ambient temperature and demolding of the mold from the PDMS substrate, d) $\mathrm{O}_{2}$ plasma to the PDMS substrate to oxidize the exposed PDMS, e) Gas-phase silanation of $1 \mathrm{H}, 1 \mathrm{H}, 2 \mathrm{H}, 2 \mathrm{H}$-perfluorodecyltrichlorosilane (PFDTS) onto the uncovered PDMS, f) Lift-off of the residual mr-I T85 in cyclohexane; $g$ ) Contact printing of thiol inks to gold substrate.

Mr-I T85 (Micro Resist) was selected as the thermoplastic resist and spin coated on the composite PDMS substrate. We chose T85, because it is an apolar polymer that has a better adhesion to the hydrophobic PDMS substrate, compared to the more commonly used PMMA. 
Integration of Capillary Force Lithography and Microcontact Printing

Three different kinds of Si templates with different feature sizes were used in CFL, one with a $500 \mathrm{~nm}$ wide cross bar motif with a $4 \mu \mathrm{m}$ period (depth $150 \mathrm{~nm}$ ), one containing high density nanoholes with half pitch down to $100 \mathrm{~nm}$ (depth $100 \mathrm{~nm}$ ) and another one containing sub-100 nanoridges ( $50 \mathrm{~nm}$ wide, $100 \mathrm{~nm}$ high, separated by $4 \mu \mathrm{m}$ ), which was fabricated by edge lithography and wet etching. ${ }^{[28]}$ Before CFL, the molds were coated with $1 H, 1 H, 2 H, 2 H$-perfluorodecyltrichlorosilane (PFDTS) as an anti-adhesion layer for better mold release. ${ }^{[29]}$

Of special interest for the fabrication of polymer patterns on flat PDMS surface is the CFL of thin (with respect to the intrusion depth of the mold) polymer layers. Thus, the amount of polymer can not fill the voids between the mold and the substrate completely, and this can result in the absence of a residual layer on the PDMS substrate surface after CFL. Thus it requires only a short oxygen plasma step to activate the uncovered PDMS surface for subsequent chemical functionalization. Reducing the oxidation time to a minimum is essential to prevent crack formation and concomitant deformation of the stamp surface. ${ }^{[30]}$ Another issue in our CFL experiments is the air permeation of the mold during the pattern transfer, since the Si molds are not gas permeable. During the CFL, the air pressure, which built up by the capillary rise of the polymers, needs to be balanced with the Laplace pressure within the close channel and there will be a small gab between the polymer and the mold. The initial spin coated film thickness was calculated to estimate the maximum height of the polymer pattern after CFL is around the half of the step height of the Si mold. Thus, there will be enough space for the air, which guarantees the air pressures will not be too high and the PDMS substrate can be successfully exposed. A $15 \mathrm{~nm}$ resist layer was spin coated when a $500 \mathrm{~nm}$ wide cross bar and $100 \mathrm{~nm}$ holes templates were used, while a $20 \mathrm{~nm}$ resist was used in the case of sub-100 nano-ridges.

\subsubsection{CFL results}

In order to get uniform patterns, before CFL, the PDMS substrate, spin-coated with T85, and the Si template were first put into the vacuum oven and pre-treated for $30 \mathrm{~min}$. The temperature was set to $150{ }^{\circ} \mathrm{C}$, which is sufficiently above the glass transition temperature $T_{g}\left(85^{\circ} \mathrm{C}\right)$ of the resist in order to ensure polymer mobility for patterning by 
CFL. After pre-heating, the Si mold was brought into conformal contact with the polymer layer on the PDMS substrate. A small weight ( $\sim 0.1$ bar) was applied on top of the Si mold to prevent separation. The substrate plus the stamp were placed under vacuum at $150{ }^{\circ} \mathrm{C}$ for the setting time to pattern the polymer layer. After cooling to ambient temperature and release of the Si mold, the patterning area and uniformity of the resulting polymer structures were examined by AFM or SEM. Figure 1 shows the AFM and SEM images of the polymer patterns on flat PDMS substrates after CFL with different molds.

In the case of the large features (500 $\mathrm{nm}$ cross bar), a grid structure according to the dimensions of the molds was formed on the PDMS surface (Figure 1a). Measuring from the AFM height image, the polymer grids have an average width of $500 \pm 10 \mathrm{~nm}$ (full width at half maximum (FWHM)) which are identical to the original mold features. The height of the polymer structures is $\sim 80 \mathrm{~nm}$ with a somewhat larger height of the crossings. Compared to the original spin coated film thickness $(15 \mathrm{~nm})$ and the fill factor of the mold $(20 \%)$ it is concluded that there is no residual film left on the contact areas of the PDMS substrate.

In the case of the high density patterns (Figure 1b), an array of slightly tapered cylinders was formed on a large area of the PDMS surface. The diameter and the height of the cylinders is $100 \pm 5$ and $\sim 45 \mathrm{~nm}$, respectively, which is in agreement with the diameters of the mold. It is clear that the polymer replication by CFL was faithful, and that the defect density is quite low over large areas. Also in this case, the original spin coated film thickness of $15 \mathrm{~nm}$ and the fill factor of the mold of $40 \%$, indicate the absence of a residual resist remaining on the contact areas after CFL.

When a sub-100 nm mold was used, polymer nanogrooves of $50 \pm 5 \mathrm{~nm}$ separated by 4 $\mu \mathrm{m}$ were formed on the PDMS surface (Figure 1c). The AFM cross-section analyses show a meniscus at the protruding end of the polymer structures. Since the distance between the nano-ridges $(4 \mu \mathrm{m})$ is much larger than the width of the ridges $(50 \mathrm{~nm})$ the resist at the contact area was easily moved to the cavities and self-organized to a U-shape structure along the side of the nanoridges. Thus, the height of the polymer film does not change significantly compared to the original spin-coated film thickness $(20 \mathrm{~nm})$. 
a)
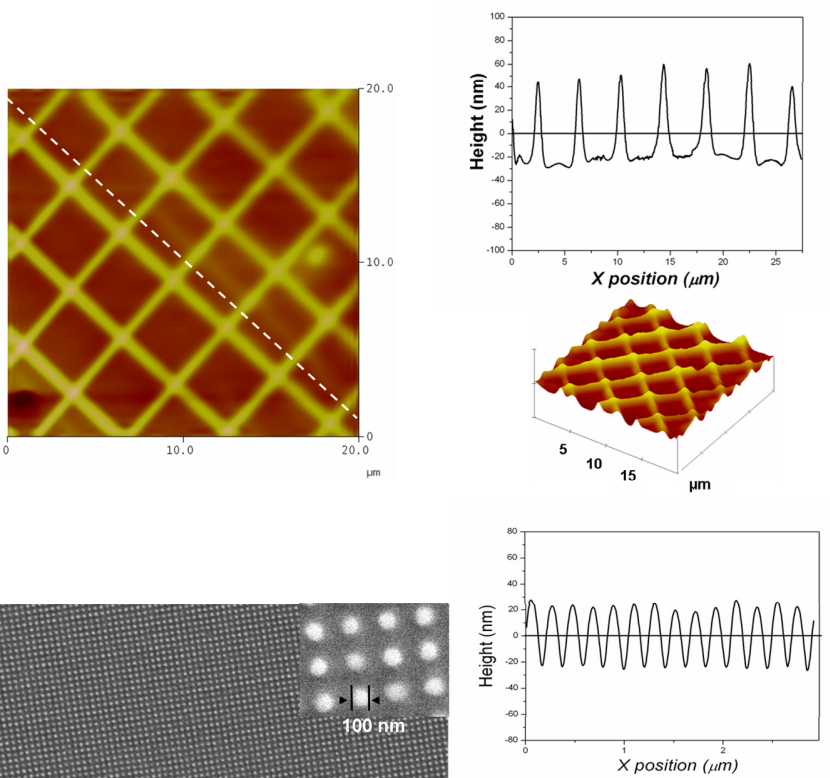

b)
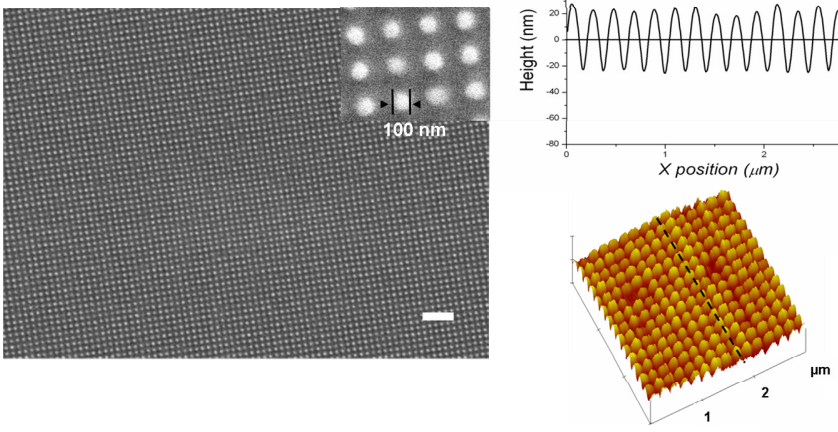

c)
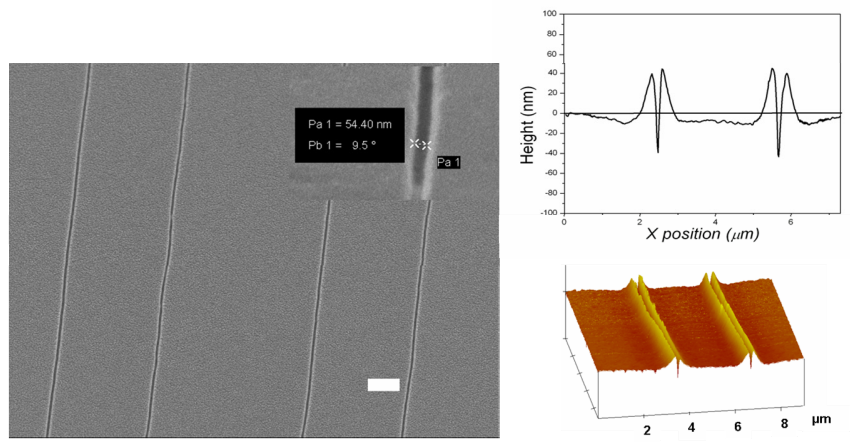

Figure 1. SEM and AFM images of different polymer patterns on flat PDMS substrates fabricated by $C F L$ at $130{ }^{\circ} \mathrm{C}$ in vacuum using a mold with: a) $500 \mathrm{~nm}$ wide grids, b) 100 $\mathrm{nm}$ slightly tapered cylinders, c) $50 \mathrm{~nm}$ grooves. The scale bar is $1 \mu \mathrm{m}$. 
This chapter presents CFL results using impermeable and non-deformable Si hard molds. The successful use of a hard mold in CFL implies that the feature size can be reduced to any that can be made on the hard mold. The CFL process times needed with the different molds have been compared. In the case of molds containing large features (500 $\mathrm{nm}$ grids), at least $5 \mathrm{~h}$ annealing time was needed to form the desired patterns and to expose the PDMS substrate. When smaller features were used (100 nm holes, $50 \mathrm{~nm}$ nanoridges), 30 min was enough to pattern the surface. This process time proves that the smaller the feature size is, the more favorable the conditions are for patterning by CFL, especially when a higher capillarity and a smaller flow distance are required.

Compared to the previous experiments using patterned flat PDMS made by regular thermal nanoimprint (NIL) (Chapter 4), CFL offers the advantage to pattern flat PDMS without applying pressure and the absence of a residual layer. Thus eliminating the need to expose the PDMS surface with RIE and shortening the PDMS oxidation step. In control experiments, regular NIL was applied to flat PDMS substrates with polymer films of the same thickness, using the same molds as in CFL. Because of the pressure applied during NIL and the low resist thickness, the soft flat PDMS surface was damaged after imprint (Figure 2). This proves that CFL is a better method to pattern such thin films on a PDMS surface compared to NIL.

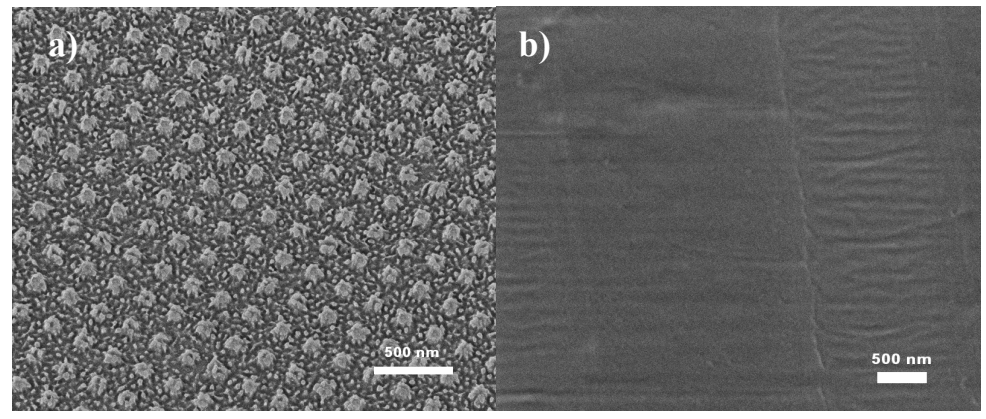

Figure 2. SEM images of different polymer patterns on flat PDMS substrates fabricated by NIL at $150^{\circ} \mathrm{C}, 2$ bar pressure: a) $100 \mathrm{~nm}$ slightly tapered cylinders, b) $50 \mathrm{~nm}$ grooves. The scale bar is $500 \mathrm{~nm}$. 
Integration of Capillary Force Lithography and Microcontact Printing

\subsubsection{Pattern transfer}

The objective was to exploit these polymer patterns as masks for the chemical functionalization of the PDMS substrate. Activation of the exposed PDMS was achieved by an oxygen plasma. Since there is no residual polymer layer left on the PDMS surface, the oxygen plasma treatment could be relatively short ( $30 \mathrm{~s}$ ), thus minimizing damage of the polymer patterns and the PDMS surface during stamp oxidation.

Immediately after $\mathrm{O}_{2}$ plasma, PFDTS was deposited from the vapor phase onto the locally oxidized PDMS substrates. The adsorbed PFDTS is assumed to react with the silanol groups present on the oxidized PDMS to yield a PFDTS-modified PDMS stamp. Subsequently, the remaining polymer resist was selectively removed by dipping the PDMS substrates into hot cyclohexane $\left(80^{\circ} \mathrm{C}\right)$ for $1 \mathrm{~min}$.

\subsection{4 $\mu \mathrm{CP}$ results}

To illustrate their applicability as stamps in soft lithography, these PFDTS-modified flat PDMS stamps were used to transfer thiol inks in both regular negative printing $((-) \mu \mathrm{CP})$ and positive printing $((+) \mu \mathrm{CP})$ experiments. In $(-) \mu \mathrm{CP}$, octadecanethiol (ODT) was transferred onto a $20 \mathrm{~nm}$ thick gold layer, followed by selective wet chemical etching of the gold in an aqueous basic solution containing $\mathrm{K}_{2} \mathrm{~S}_{2} \mathrm{O}_{3} / \mathrm{K}_{3} \mathrm{Fe}(\mathrm{CN})_{6} / \mathrm{K}_{4} \mathrm{Fe}(\mathrm{CN})_{6}{ }^{[31]}$ Figure 3a-c shows SEM images of gold patterns generated by (-) $\mu \mathrm{CP}$ using PFDTS-modified flat PDMS stamps and subsequent wet etching.

When using the $500 \mathrm{~nm}$ grid structure (Figure 3a), the gold patterns had a uniform width of $480 \pm 30 \mathrm{~nm}$, which closely matches the original Si mold. In case of the dot patterns (Figure 3b), the gold dots had a diameter of $80 \pm 15 \mathrm{~nm}$. The slight decrease (10 $\mathrm{nm}$ per side) compared to the original mold is attributed to the isotropic wet etching of the gold. ${ }^{[3 l]}$ With sub-100 nm trenches in gold, we found that a longer wet etching time was necessary to achieve a clear contrast. After etching, nanotrenches in gold were formed with a width of $100 \pm 25 \mathrm{~nm}$ (Figure 3c).

In order to get high quality gold lines, $(+) \mu \mathrm{CP}$ was performed by using a thioethermodified poly(propylene imine) dendrimer $(\mathrm{G} 3-\mathrm{M})^{[32]}$ as the ink that is compatible with the requirements of $\mu \mathrm{CP}$, but does not form etch-blocking self-assembled monolayers (SAMs). 
Adsorption of ODT from solution to the non-contacted areas and subsequent wet chemical etching yielded the positive patterns. High quality nanolines were formed after gold etching (Figure 3d) with an average size of $80 \pm 15 \mathrm{~nm}$. The slight increase of the width of the gold lines is again attributed to the wet etching step.
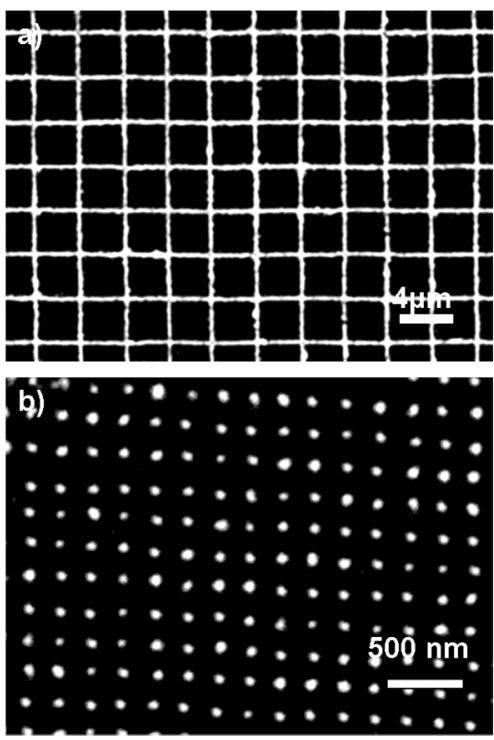
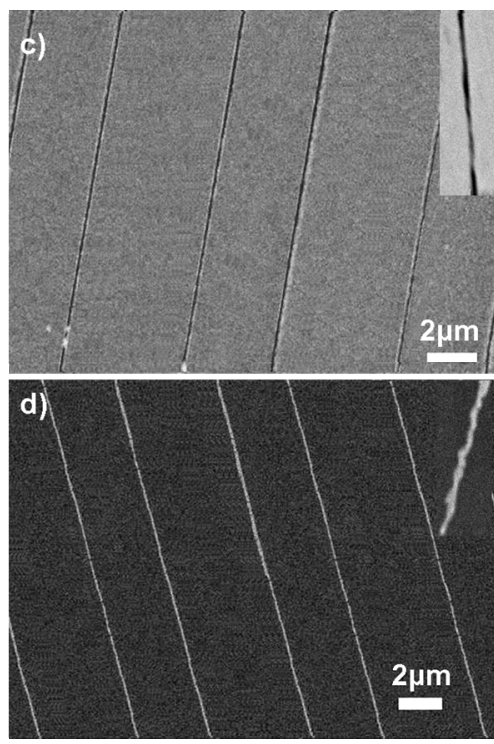

Figure 3. SEM images of different structures generated in a $20 \mathrm{~nm}$ gold layer by application of $(-) \mu C P(a, b, c)$ and $(+) \mu C P(d)$ using the PFDTS-modified flat PDMS stamps made by CFL. Light regions are gold, and dark regions are the underlying $\mathrm{Si} / \mathrm{SiO}_{2}$ substrate. a) $\sim 500 \mathrm{~nm}$ wide gold grids fabricated by (-) $\mu C P$ with a stamp printed by 0.1 mM ODT for $60 \mathrm{~s}$, followed by selective wet chemical etching of gold for $7 \mathrm{~min}$. b) $\sim 80 \mathrm{~nm}$ dots of gold on slicon fabricated by (-) $\mu \mathrm{CP}$ (same conditions). c) array of $\sim 95 \mathrm{~nm}$ wide trenches in gold fabricated by (-) $\mu C P$ with a stamp printed with the same conditions, followed by selective wet chemical etching of gold for 8 min. d) array of $\sim 85 \mathrm{~nm}$ wide gold lines fabricated by $(+) \mu C P$ with a stamp printed by $4 \times 10^{-2} \mathrm{mM}$ G3-M poly(propylene imine) dendrimer ink for $10 \mathrm{~s}$, followed by a $5 \mathrm{~s}$ dip in $0.1 \mathrm{mM}$ ODT solution, and selective wet chemical etching of gold for $2 \mathrm{~min}$.

\subsection{Conclusions}

In summary, a novel nanopatterning method by combining $\mathrm{CFL}$ and $\mu \mathrm{CP}$ was developed. High-resolution CFL was achieved by using patterned hard $\mathrm{Si}$ wafers as the 
Integration of Capillary Force Lithography and Microcontact Printing

mold and flat PDMS as the substrate. The formed polymer pattern was subsequently used as a mask for the chemical functionalization of the PDMS stamps. These flat stamps are mechanically stable and the chemical patterns on their surfaces allow one to control where ink transport takes place, on the sub-100 $\mathrm{nm}$ to many $\mu \mathrm{m}$ length scale. The use of flat PDMS as the substrate during CFL as well as the stamp in $\mu \mathrm{CP}$ increases the resolution of both methods. The combined CFL- $\mu \mathrm{CP}$ procedure provides a convenient and inexpensive method to create high resolution patterns.

\subsection{Experimental}

\section{Materials and methods:}

All experiments were carried out in a regular chemical laboratory. Octadecanethiol (ODT) was purchased from Aldrich. $1 H, 1 H, 2 H, 2 H$-Perfluorodecyltrichlorosilane (PFDTS, purity 97\%), was purchased from ABCR GmbH. Imprint resist mr-I T85 was obtained from Micro Resist. All materials were used as received without further purification. The third generation thioether-modified poly(propylene imine) dendrimer (G3-M) was synthesized according to a reported procedure ${ }^{[32]}$ A kit of PDMS prepolymer (Sylgard 184 silicon elastomer) and PDMS curing agent was purchased from Dow Corning Corporation. Gold layers $(20 \mathrm{~nm})$ on titanium-primed $(2-3 \mathrm{~nm})$ silicon wafers were purchased from Ssens BV (Hengelo, The Netherlands).

\section{Composite flat PDMS substrates}

Composite flat PDMS substrates ${ }^{[26]}$ were prepared by first casting the liquid prepolymer of the elastomer onto a polished $\mathrm{Si}$ wafer onto which an anti-adhesion layer had been deposited. After degassing, a piece of cover glass (MENZEL-GLÄSER BB015015A1 $15 \mathrm{~mm} \times 15 \mathrm{~mm})$ which has been cleaned by a piranha solution (3:1 mixture of sulfuric acid and hydrogen peroxide. CAUTION! Piranha solutions should be handled with great care in open containers in a fume hood. Piranha is highly corrosive and toxic and potentially explosive) was then put into the uncured mixture. The cover glass sank to the bottom of the PDMS liquid leaving a thin layer between the cover glass and the Si wafer. After curing at $60{ }^{\circ} \mathrm{C}$ for $8 \mathrm{~h}$ the stamp was peeled off from the $\mathrm{Si}$ wafer.

\section{CFL procedure}


A thin film of mr-I T85 solution was spin-coated onto the PDMS composite substrates, followed by a soft baking step for $2 \mathrm{~min}$ at $140{ }^{\circ} \mathrm{C}$ on a hot plate.

Molds with silicon nanoridges were fabricated according to a published procedure. ${ }^{[29]}$ The other Si molds were purchased from NIL Technology, one with a $500 \mathrm{~nm}$ cross bar and a $4 \mu \mathrm{m}$ period (depth $150 \mathrm{~nm}$ ), and another one containing holes with half pitch down to $100 \mathrm{~nm}$ (depth $100 \mathrm{~nm}$ ). PFDTS was used as an anti-adhesion layer to facilitate the stampsubstrate separation.

First, the PDMS substrate coated with mr-I T85 and the Si template were equilibrated at the annealing temperature for $30 \mathrm{~min}$. Thereafter, the $\mathrm{Si}$ mold was brought into conformal contact with the polymer layer on the PDMS substrate. A small weight ( $\sim 0.1$ bar) was applied on top of the Si molds. The system was left at $150{ }^{\circ} \mathrm{C}$ in vacuum for $5 \mathrm{~h}$ in case of $500 \mathrm{~nm}$ grids and $30 \mathrm{~min}$ in case of $100 \mathrm{~nm}$ holes and $50 \mathrm{~nm}$ nanoridges were used. After cooling to $50{ }^{\circ} \mathrm{C}$ for $10 \mathrm{~min}$, the template was separated from the PDMS substrate.

\section{Pattern transfer}

After CFL, $\mathrm{O}_{2}$ plasma (10 mTorr, $10 \mathrm{~W}, 20 \mathrm{sccm} \mathrm{O}_{2}$ ) was applied for $30 \mathrm{~s}$ on the polymer patterned PDMS substrate to activate the exposed PDMS. Directly after $\mathrm{O}_{2}$ plasma, PFDTS was immediately deposited for $30 \mathrm{~min}$ from vapor phase onto the locally oxidized PDMS substrates. The adsorbed PFDTS was assumed to react with the silanol groups present on the oxidized PDMS to yield a PFDTS-modified chemically patterned PDMS stamp. ${ }^{[22]}$ The substrates were thereafter cured in an oven for $3 \mathrm{~h}$ at $60{ }^{\circ} \mathrm{C}$ to complete the reaction.

Subsequently, the remaining polymer resist was selectively removed by dipping the PDMS substrates into hot cyclohexane $\left(80^{\circ} \mathrm{C}\right)$ for $1 \mathrm{~min}$.

\section{Contact printing}

Before printing, gold substrates were treated with oxygen plasma, immersed in ethanol for $1 \mathrm{~h}$, rinsed with water (Millipore) and ethanol, and dried with nitrogen.

The stamps were inked with a few drops of ODT solution in ethanol $(0.1 \mathrm{mM})$ and dried in a stream of nitrogen. After drying the stamps with nitrogen, the stamps were placed manually on the substrate, and were pressed slightly against the gold surface at the initial stage of the printing to induce conformal contact. The printing time was $1 \mathrm{~min}$ and the gold 
Integration of Capillary Force Lithography and Microcontact Printing

samples were selectively etched by keeping the substrate in $\mathrm{S}_{2} \mathrm{O}_{3}{ }^{2-} /$ ferri/ferrocyanide-based etching solutions for different times (in case of features $>100 \mathrm{~nm}, 7$ min etching is used; in the case of sub-100 nm nano-grooves, 8 min etching is needed to get a clear contrast).

In $(+) \mu \mathrm{CP}$ of the G3-M ink ${ }^{[32]}$, the stamp was inked with a solution of G3-M $\left(4 \times 10^{-2}\right.$ $\mathrm{mM}$ ). Printing was performed for $2 \mathrm{~min}$, and the substrate was subsequently dipped in a 0.1 $\mathrm{mM}$ ODT solution for $5 \mathrm{~s}$. A freshly prepared etching solution consisting of $10 \mathrm{mM}$ $\mathrm{Fe}\left(\mathrm{NO}_{3}\right)_{3}, 15 \mathrm{mM}$ thiourea and $1.2 \% \mathrm{HCl}$ was used to etch the dendrimer-printed areas.

\section{Measurements}

AFM analyses were carried out with a NanoScope $\amalg$ (Veeco/Digital Instruments, Santa Barbara, CA, USA) Multimode Atomic Force Microscope equipped with a J-scanner, in tapping mode by using $\mathrm{Si}_{3} \mathrm{~N}_{4}$ cantilevers (Nanoprobes, Veeco/Digital Instruments) with a nominal spring constant of about $0.32 \mathrm{Nm}^{-1}$. AFM imaging was performed at ambient conditions.

Low-resolution SEM imaging was carried out with a JEOL, JSM-5610 SEM operating in secondary electron detection mode. High-resolution SEM imaging was carried out with a JEOL Gemini 1550 FEG-SEM.

\subsection{References}

[1] S. J. Holmes, P. H. Mitchell, M. C. Hakey, IBM J. Res. Dev. 1997, 41, 7.

[2] R. H. Stulen, D. W. Sweeney, IEEE J. Quantum Electronics 1999, 35, 694.

[3] J. P. Silverman, J. Vac. Sci.Technol. B 1997, 15, 2117.

[4] M. A. McCord, J. Vac. Sci.Technol. B 1997, 15, 2125.

[5] U. S. Tandon, Vacuum 1992, 43, 241.

[6] Y. N. Xia, G. M. Whitesides, Angew. Chem. Int. Ed. 1998, 37, 551.

[7] A. Kumar, G. M. Whitesides, Appl. Phys. Lett. 1993, 63, 2002.

[8] Y. N. Xia, E. Kim, G. M. Whitesides, Chem. Mater. 1996, 8, 1558.

[9] E. Kim, Y. N. Xia, G. M. Whitesides, Nature 1995, 376, 581.

[10] Y. N. Xia, G. M. Whitesides, Langmuir 1997, 13, 2059.

[11] X. M. Zhao, A. Stoddart, S. P. Smith, E. Kim, Y. Xia, M. Prentiss, G. M. Whitesides, Adv. Mater. 1996, 8, 420. 
[12] E. Kim, Y. N. Xia, X. M. Zhao, G. M. Whitesides, Adv. Mater. 1997, 9, 651.

[13] L. Libioulle, A. Bietsch, H. Schmid, B. Michel, E. Delamarche, Langmuir 1999, 15,300 .

[14] Y. N. Xia, G. M. Whitesides, Annu. Rev. Mater. Sci. 1998, 28, 153.

[15] K. Y. Suh, Y. S. Kim, H. H. Lee, Adv. Mater. 2001, 13, 1386.

[16] K. Y. Suh, H. H. Lee, Adv. Func. Mater. 2002, 12, 405.

[17] X. Y. Lei, L. Wu, P. Deshpande, Z. N. Yu, W. Wu, H. X. Ge, S. Y. Chou, Nanotechnology 2003, 14, 786.

[18] B. Michel, A. Bernard, A. Bietsch, E. Delamarche, M. Geissler, D. Juncker, H. Kind, J. P. Renault, H. Rothuizen, H. Schmid, P. Schmidt-Winkel, R. Stutz, H. Wolf, IBM J. Res. Dev. 2001, 45, 697.

[19] A. Bietsch, B. Michel, J. Appl. Phys. 2000, 88, 4310.

[20] K. G. Sharp, G. S. Blackman, N. J. Glassmaker, A. Jagota, C. Y. Hui, Langmuir 2004, 20, 6430 .

[21] Y. G. Y. Huang, W. X. Zhou, K. J. Hsia, E. Menard, J. U. Park, J. A. Rogers, A. G. Alleyne, Langmuir 2005, 21, 8058.

[22] R. B. A. Sharpe, D. Burdinski, J. Huskens, H. J. W. Zandvliet, D. N. Reinhoudt, B. Poelsema, J. Am. Chem. Soc. 2005, 127, 10344.

[23] E. Delamarche, C. Donzel, F. S. Kamounah, H. Wolf, M. Geissler, R. Stutz, P. Schmidt-Winkel, B. Michel, H. J. Mathieu, K. Schaumburg, Langmuir 2003, 19, 8749 .

[24] Z. Zheng, J. W. Jang, G. Zheng, C. A. Mirkin, Angew. Chem. Int. Ed. 2008, 47, 9951.

[25] X. X. Duan, V. B. Sadhu, A. Perl, M. Peter, D. N. Reinhoudt, J. Huskens, Langmuir 2008, 24, 3621.

[26] X. X. Duan, Y. P. Zhao, A. Perl, E. Berenschot, D. N. Reinhoudt, J. Huskens, Adv. Mater. 2009, 21, 2798.

[27] H. Yoon, T. I. Kim, S. J. Choi, K. Y. Suh, M. J. Kim, H. H. Lee, Appl. Phys. Lett. 2006, 88 .

[28] Y. Zhao, E. Berenschot, M. de Boer, H. Jansen, N. Tas, J. Huskens, M. 
Elwenspoek, J. of Micromech. Microeng. 2008, 18, 064013.

[29] G. S. Ferguson, M. K. Chaudhury, H. A. Biebuyck, G. M. Whitesides, Macromolecules 1993, 26, 5870.

[30] M. J. Owen, P. J. Smith, J. Adhes. Sci. Technol. 1994, 8, 1063.

[31] Y. N. Xia, X. M. Zhao, E. Kim, G. M. Whitesides, Chem. Mater. 1995, 7, 2332.

[32] A. Perl, M. Peter, B. J. Ravoo, D. N. Reinhoudt, J. Huskens, Langmuir 2006, 22, 7568 . 



\section{Chapter 6}

\section{Large-area Nanoscale Patterning of Functional Materials by Nanomolding in \\ Capillaries*}

Within the past years there has been much effort in developing and improving new techniques for the nanoscale patterning of functional materials used in promising applications like nano(opto)electronics. Here a high-resolution soft lithography technique nanomolding in capillaries (NAMIC) is demonstrated. First, a composite PDMS stamp with sub-100 nm features is fabricated as a nanomold for NAMIC by nanoimprint lithography. Then, NAMIC has been used to pattern different functional materials such as fluorescent dyes, proteins, nanoparticles, thermoplastic polymers, and conductive polymers at the nanometer scale over large areas. These results show that NAMIC is a simple, versatile, low-cost, and high-throughput nanopatterning tool.

* Part of this chapter has been submitted for publication: X. X. Duan, Y. P. Zhao, E. Berenschot, N. R. Tas, D. N. Reinhoudt, J. Huskens, "Large-area Nanoscale Patterning of Functional Materials by Nanomolding in Capillaries" 


\subsection{Introduction}

Major efforts in nanotechnology are devoted to the patterning of functional materials such as fluorescent compounds, biomolecules, nanoparticles, and polymers, into size and shape controlled structures for use in applications such as electronics, optoelectronics, photonics, and sensing. ${ }^{[1-4]}$ The continuing development of lithography technologies allows patterns to be produced with feature sizes well below $100 \mathrm{~nm}$. With the increase of materials used for functional structures, there is a high demand for low-cost and large-area manufacturing techniques, both for real applications in actual devices, but definitely also for rapid prototyping in research environments. Soft-lithography, ${ }^{[5,6]}$ which is a collective term for a number of non-photolithographic techniques, fulfills these requirements, to some extent, by avoiding the use of cleanroom facilities. Specifically, micromolding in capillaries (MIMIC) ${ }^{[7]}$ which is a solution-based technique, is a potentially cheap, simple, and versatile tool that has received much attention in recent years. ${ }^{[8]}$ In a typical MIMIC scheme, a poly(dimethylsiloxane) stamp with parallel protrusions is placed in contact with a smooth surface, so that the grooves form channels (capillaries). When a solution is poured at the open end of the stamp, the liquid spontaneously fills the channels by the capillary pressure. As the solution volume gradually shrinks because of solvent evaporation, the capillary forces drive the formation of a meniscus under the roof of the stamp channels. After the complete evaporation of the solvent, the stamp is gently removed leaving the patterns on the surface. Due to the mechanical instability of the soft polymeric stamp, ${ }^{[9,10]}$ the dimensions of the channels in MIMIC are typically limited to about a few hundred nanometers. ${ }^{[11-13]}$ It is possible to fabricate narrower patterns by diluting the solutions used during MIMIC. However, when most of the solvent has evaporated, the residual solutions tend to accumulate and aggregate on the boundaries of the channel and cause dewetting, thus giving rise to defects in the stripes or form split lines. ${ }^{[14,15]}$

In this chapter, a new, wet, lithographical approach is introduced, nanomolding in capillaries (NAMIC), to pattern different functional materials from their solutions at sub$100 \mathrm{~nm}$ dimensions. The major improvement is the use of a hybrid stamp with harder, more well-defined features which was fabricated by nanoimprint lithography (NIL) on flat PDMS, 
thus allowing nanochannel formation and facilitating nanoscale surface patterning. NAMIC can create large area well-defined sub-100 nm patterns (according to the dimensions of the NIL mold) in a relatively short time and it can be used to pattern a large variety of materials (fluorescent dye, nanoparticles, (bio)molecules, polymers) from their solutions, which is difficult by other nanofabrication methods.

\subsection{Results and discussion}

\subsubsection{Fabrication scheme}

Scheme 1 shows a schematic outline of the NAMIC process. It involves the fabrication of a hybrid stamp by nanoimprint lithography (NIL) with a thin film of thermal resist on a composite flat PDMS substrate and the soft bonding of this stamp to the $\mathrm{Si} / \mathrm{SiO}_{2}$ or glass substrates by oxygen plasma treatment to form a parallel array of nanochannels. The channels are then filled with a solution containing functional materials. After evaporation of the solvent and detachment of the mold, the nanopatterned materials are deposited on the substrate.

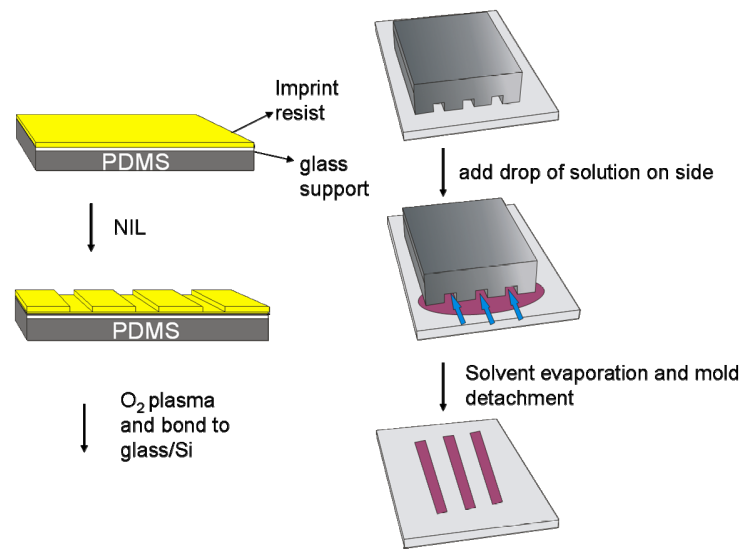

Scheme 1. Process scheme for nanomolding in capillaries (NAMIC).

In order to achieve high lateral resolution molding, it requires small features with high conformational stability, but without compromising the necessary conformal contact to allow closed channel formation upon contacting a substrate. Chapter 3 has reported the 
surface patterning of a thin layer of a thermoplastic polymer on a flat PDMS substrate by nanoimprint lithography. ${ }^{[16]}$ First, a composite flat PDMS substrate was made by curing commercial PDMS (Sylgard 184) with a cover glass as a support layer, which has been described in Chapter 4. The advantage of this method is that the thin glass gives the stamp enough support during imprint, while the composite stamp has enough flexibility to achieve good conformal contact with a substrate.

\subsubsection{Nanomold fabrication}

Thermoplastic polymer mr-I T85 (Micro Resist) was used as imprint resist and spincoated on the flat composite PDMS substrate. T85 was chosen, because it is an apolar polymer which has a better adhesion to the hydrophobic PDMS substrate, compared to the more commonly used PMMA. Si molds with different sizes of nanoridges (50 or $100 \mathrm{~nm}$ wide, $100 \mathrm{~nm}$ high, separated by $4 \mu \mathrm{m}$ ) were used as imprint templates. ${ }^{[17]}$ The imprinting was performed under mild conditions $\left(150^{\circ} \mathrm{C}\right.$ and 2 bar $)$.

Polymer nanogrooves of 50 or $100 \pm 5 \mathrm{~nm}$ separated by $4 \mu \mathrm{m}$ were formed on the PDMS surface. Figure 1 shows the SEM images of the polymer grooves on the flat PDMS substrate with cross sections of the imprinted polymer films. A typical length of the PDMS mold was approximately 0.5 to $1.5 \mathrm{~cm}$, and the width of the mold was 0.5 to $2 \mathrm{~cm}$, which can contain about 1250 to 5000 parallel channels.
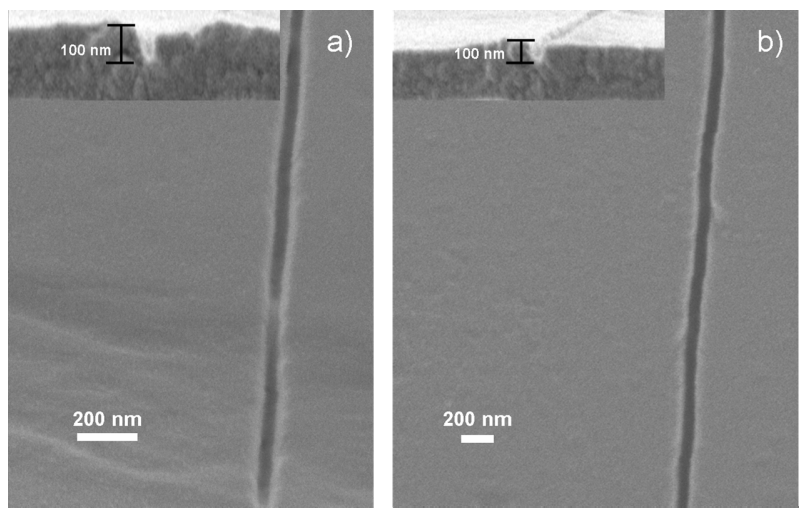

Figure 1. SEM images of T85 polymer grooves on PDMS of (a) $50 \mathrm{~nm}$ wide, $100 \mathrm{~nm}$ deep and (b) $100 \mathrm{~nm}$ wide, $100 \mathrm{~nm}$ deep, fabricated by NIL. 


\subsubsection{NAMIC procedure}

Crucial steps for ensuring complete sealing and easy separation between the nanomold and the substrate before NAMIC are: (1) a short oxygen plasma step to pretreat the mold surface to promote adhesion upon contacting the substrate (needed because of the low surface energy of $\mathrm{T} 85^{[18,19]}$ ), and (2) washing of the substrate with a piranha solution $\left(\mathrm{H}_{2} \mathrm{O}_{2}: \mathrm{H}_{2} \mathrm{SO}_{4}=1: 3\right.$ by volume $)$ to create a thin and smooth native oxide layer. Thereafter, the mold was placed on the substrate, and an array of nanochannels was formed. Since there is only a thin layer of T85 (300 nm) on top of the mold and the thick PDMS backbone remains soft, good contact was achieved between the mold and the substrate. For good sealing, a few drops of water were applied immediately at the edges of the nanochannels. The water filling the channels generates a negative pressure and thus assists the sealing. Under these conditions, the mold can be peeled off after NAMIC. No leakage of materials from the channels was observed which is confirmed by the patterning results (see below).

After the formation of the nanochannels, a drop of a solution or suspension containing a fluorescent dye, proteins, $\mathrm{SiO}_{2}$ nanoparticles, or a polymer was placed at one end of the channels. Since the dimensions of the channels are very small $(100 / 50 \times 100 \mathrm{~nm})$, the capillary pressure becomes very high and the fluid fills the channels by capillary action only ${ }^{[20]}$ This was witnessed by the observed high rates of filling: typically the $\mathrm{cm}$-long channels were filled within seconds. Once the channels were filled completely, the solvent was allowed to evaporate at room temperature and the materials precipitated out onto the substrate within the confinement of the channels. Once the solvent was completely evaporated, the PDMS mold was carefully peeled off the substrate, leaving nanometric stripes on the substrate. Occasionally, traces of residual T85 were observed on the substrate due to the oxygen plasma pre-treatment of the nanomolds, but these could be easily removed by rinsing with an apolar solvent (e.g. cyclohexane, xylene) or by oxygen plasma in the case of patterning with particles.

The big advantage of the NAMIC approach compared with MIMIC and other conventional patterning methods is that within a single step, it can create large area welldefined sub-100 nm patterns (according to the dimensions of the nanomold) in a relatively 
short time and it can be used to pattern a large variety of materials from their solutions as long as they can dissolve in a polar solvent, which does not swell the nanomold.

\subsubsection{Patterning dyes by NAMIC}

As a first example, tetramethylrhodamine isothiocyanate (TRITC) dye in aqueous solution $(10 \mu \mathrm{M})$ was deposited by NAMIC through a $50 \times 100 \mathrm{~nm}(\mathrm{w} \times \mathrm{h})$ nanomold onto a $\mathrm{Si} / \mathrm{SiO}_{2}$ substrate. After water evaporation, the TRITC dye precipitated onto the substrate. The mold was peeled off leaving the TRITC patterns on the surface. The silicon substrate was then rinsed with cyclohexane. Figure 2 shows fluorescence microscopy and AFM height images of the TRITC patterns on the silicon substrates.
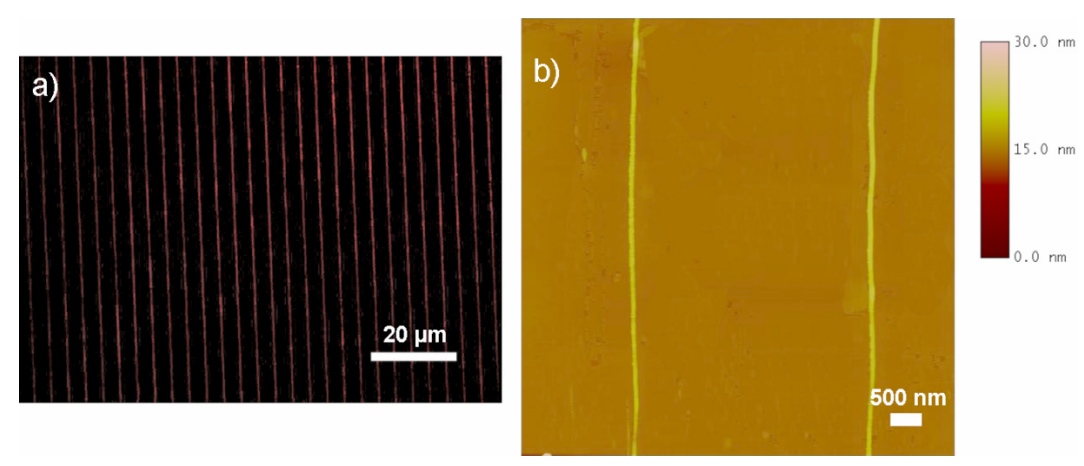

Figure 2. Fluorescence micrograph (a) and AFM height image (b) of TRITC dye nanopatterns fabricated on $\mathrm{Si}_{/} \mathrm{SiO}_{2}$ by NAMIC using a $50 \times 100 \mathrm{~nm}(\mathrm{w} \times$ h) mold.

As observed in the optical and AFM images, the TRITC dye formed continuous nanolines over large areas without obvious defects. The absence of fluorescence between the patterned nanolines indicates that no dyes are deposited in the contact areas thus confirming the good sealing of the nanochannels. AFM indicates the cleanliness of the substrate between the nanolines. Measuring from the AFM height image (Figure 2b), the nanolines have an average width of $55 \pm 5 \mathrm{~nm}$ and a height of $8 \pm 6 \mathrm{~nm}$. The width compares well to the width of the nanomold $(50 \mathrm{~nm})$. The non-uniform fluorescence intensity as seen from the optical image (Figure $2 \mathrm{a}$ ) and relatively large spread in the height 
Large-area Nanoscale Patterning of Functional Materials by Nanomolding in Capillaries $(8 \pm 6 \mathrm{~nm})$ are probably due to the low concentration of the TRITC dye or different evaporation rates at different areas within the nanochannels.

\subsubsection{Patterning proteins by NAMIC}

The ability to spatially orient and anchor biomolecules at the nanoscale affords useful materials for biosensors, and tissue engineering applications. ${ }^{[21]}$ The possibility to work in water extends NAMIC to pattern biomolecules at the nanoscale in a simple approach. Fluorescein (FITC)-conjugated ChromPure Human IgGs in buffer solution (1 mg/ml) were deposited by NAMIC through a $100 \times 100 \mathrm{~nm}$ nanomold onto a glass substrate. Figure 3 shows fluorescence microscopy and AFM 3D images of the IgGs patterns on the glass substrates.
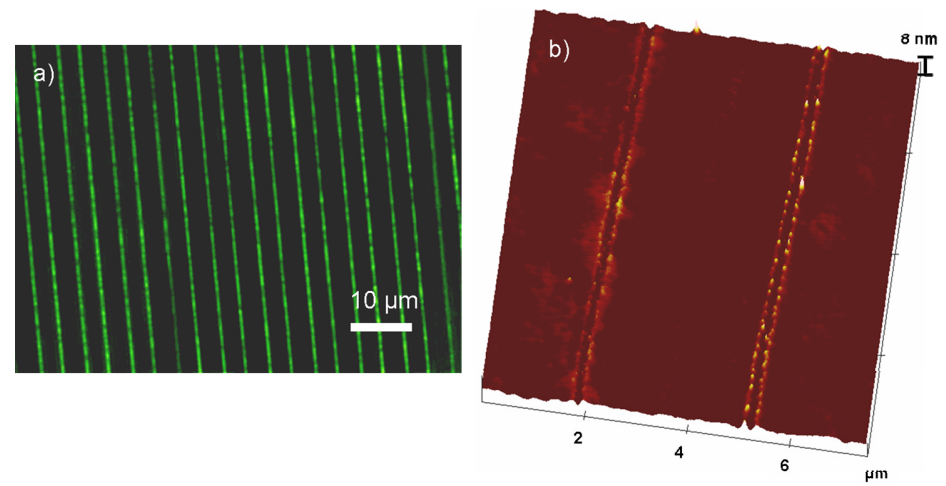

Figure 3. Fluorescence micrograph (a) and 3D AFM image (b) of FITC-labeled human IgG patterns fabricated on glass by NAMIC using a $100 \times 100 \mathrm{~nm}$ mold.

As seen from the fluorescence image (Figure 3a), IgGs were successfully deposited on the glass surface by NAMIC. AFM (Figure 3b) shows the IgGs formed dots patterns which are perfectly aligned along the edges of the channel. The average size of the dots is $25 \pm 2$ $\mathrm{nm}$ and thickness is about 4-5 nm. As reported before, antibodies (in solution) are described as disks of 10 to $14 \mathrm{~nm}$ in diameter and a few nanometers thick. ${ }^{[22]}$ It suggests that the dot patterns formed along the channels are aggregates of a few protein molecules and thus that, NAMIC can potentially be a method of choice to pattern and study single protein molecules on surfaces. 


\subsubsection{Patterning silica nanoparticles (NPs) by NAMIC}

Processing and patterning nanoparticles (NPs) into regular arrays on a surface is important for many technological applications. With standard top-down microfabrication techniques, it is difficult and time-consuming to create sparse patterns of small particles. ${ }^{[23]}$ In contrast, NAMIC is commensurate to nanoscopic particle sizes and thus offers a simple and efficient way to surface patterning of NPs.

Here, $\mathrm{SiO}_{2}$ NPs with a $60 \mathrm{~nm}$ diameter in water $(1 \mathrm{mg} / \mathrm{l})$ were deposited on a silicon substrate by NAMIC using a $100 \times 100 \mathrm{~nm}$ cross section nanomold. Spatially organized arrays of single particle wide lines of silica NPs were successfully fabricated, exploiting the self-organization and dewetting of the NPs. Figure 4 shows the SEM and 3D AFM height images of the $60 \mathrm{~nm} \mathrm{SiO} 2$ NPs patterns on the silicon substrate.
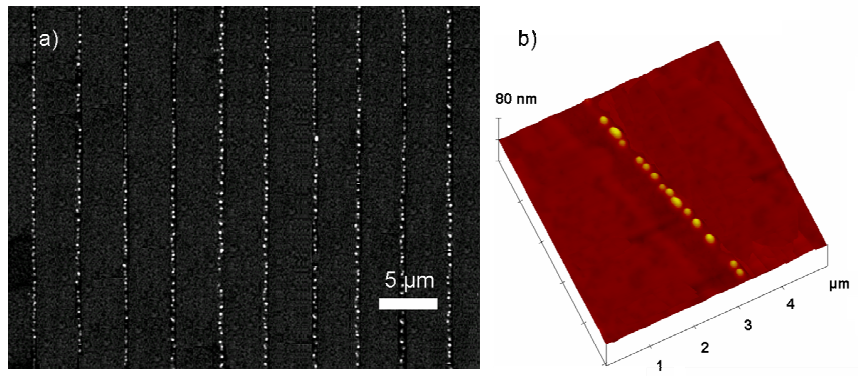

Figure 4. SEM (a) and 3D AFM (b) images of $60 \mathrm{~nm}$ silica nanoparticles assembled on $\mathrm{Si} / \mathrm{SiO}_{2}$ by NAMIC using a $100 \times 100 \mathrm{~nm}$ mold.

As seen from the SEM and AFM images, homogeneous single particle lines were formed at large scales on the substrate with few defects. The particles have an average height of $60 \pm 10 \mathrm{~nm}$ which indicates the absence of particle aggregation within the nanochannels. The particles did not form continuous lines and the average spacing between the individual NPs was $500 \pm 120 \mathrm{~nm}$ as measured from the SEM image (Figure 4a). Attempts to increase the concentration of the particle solution led to increase of the viscosity, incomplete filling of the channels before solvent evaporation, and occasional aggregation of the nanoparticles inside the nanochannels leading to clogging. Further 
Large-area Nanoscale Patterning of Functional Materials by Nanomolding in Capillaries studies may involve fine-tuning the size of the nanoparticles and the dimensions of the nanochannels combined with different chemical functionalities of the particles and substrates to employ directed assembly of the NPs within the 2D confined nanochannels.

\subsubsection{Patterning polymers by NAMIC}

Another important application of molding in capillaries is to fabricate continuous polymer lines on various substrates in a single step such as polymer precursors ${ }^{[7,24]}$ and conductive polymers. ${ }^{[11,12,25,26]}$ However, up to date, pattern sizes were all on the micron scale due to the limited mechanical stability of the soft mold. Here sub-100 nm polymer patterns at large areas are shown using NAMIC. First, a commercially available thermoplastic polymer mr-7010 (Micro Resist) was deposited through a $50 \times 100 \mathrm{~nm}(\mathrm{w} \times$ h) nanomold on a silicon substrate by NAMIC. Figure 5 shows the optical microscope, SEM, and 3D AFM height images of the polymer patterns.
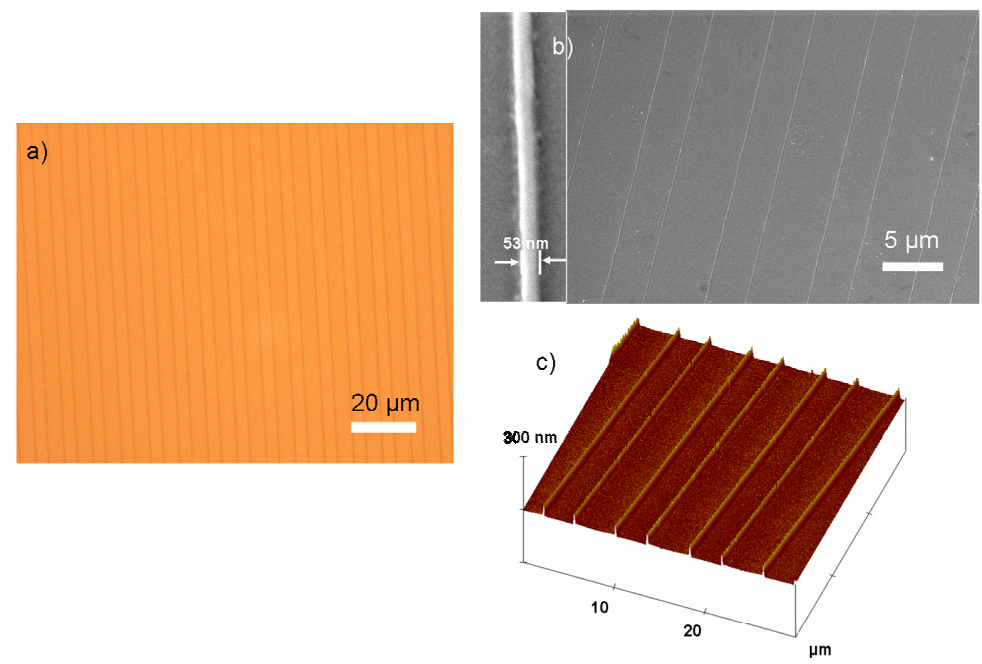

Figure 5. Optical microscopy (a), SEM (b), and 3D AFM height (c) images of thermoplastic polymer mr-7010 patterns fabricated on $\mathrm{Si} / \mathrm{SiO}_{2}$ by $\mathrm{NAMIC}$ using a $50 \times 100$ $n m(w \times h)$ mold.

As seen from Figure 5, mr-7010 formed continuous nanowires over large areas without obvious defects, and the lengths of the patterned polymer wires can extend to $\mathrm{cm}$ 
dimensions. As measured from the SEM and AFM height images (Figure 5b,c) the nanowires have an average width of $55 \pm 3 \mathrm{~nm}$ and a height of $50 \pm 5 \mathrm{~nm}$. Again, the width corresponds well to the width of the nanomold $(50 \mathrm{~nm})$ and the height corresponds to filling half of the height of the channels.

Conductive polymers are being used to replace both conventional metals and inorganic materials in developing nano(opto)electronic devices. Among them, poly(3,4ethylenedioxythiophene) doped with poly(styrene sulfonate) (PEDOT-PSS) (Figure 6a), available as an aqueous suspension, is one of the most promising electrode materials for organic devices owing to its high conductivity, high visible-light transmissivity, and excellent stability. ${ }^{[27]}$ Downscaling of patterns of PEDOT-PSS, or similar classes of conducting polymers, is therefore of high priority in the future development of organic nanoelectronics. Here PEDOT-PSS nanowires were fabricated on $\mathrm{SiO}_{2}$ substrates from their aqueous solutions $(\sim 1.3 \mathrm{wt} \%)$ through a $100 \times 110 \mathrm{~nm}$ nanomold. Figure 6 shows the POM , SEM, and AFM height images of the PEDOT-PSS patterns

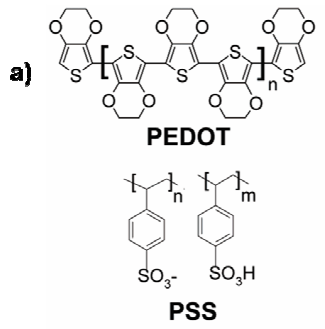

b)
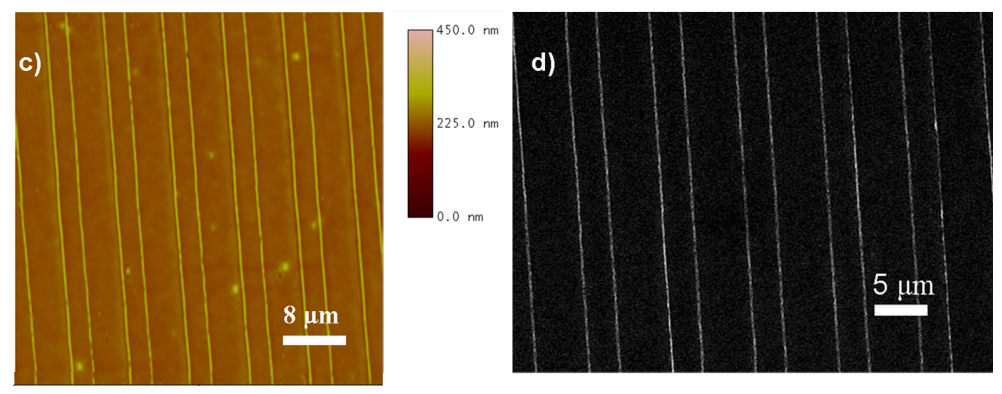

Figure 6. Molecular structure of conductive polymer mixture PEDOT-PSS (a), POM (b) AFM height (c) and SEM (d) images of PEDOT-PSS nanowires fabricated on $\mathrm{Si} / \mathrm{SiO}_{2}$ (with $300 \mathrm{~nm}$ thermal oxide on top) by NAMIC using a $100 \times 110 \mathrm{~nm}$ mold. 
Large-area Nanoscale Patterning of Functional Materials by Nanomolding in Capillaries

As seen in Figure 6, the PEDOT-PSS formed continuous nanowires over large areas (over several square centimeters) and since PEDOT is conductive it shows a high contrast in the SEM image without further metal coating. As measured from the SEM and AFM height images (Figure 6c,d), the nanowires have an average width of $100 \pm 5 \mathrm{~nm}$ and $90 \pm 5$ $\mathrm{nm}$ in height. These relatively high polymer patterns was achieved by putting extra drops of PEDOT-PSS solutions after water evaporation within the channels to get thicker conductive wires thus facilitating the conductivity measurements. The length of the patterned nanowires is extending to $\mathrm{cm}$ resulting in connected PEDOT lines with a length-to-width ratio exceeding 100,000 .

\subsubsection{Conductivity measurement of the PEDOT nanowires}

In order to measure the resistance of the PEDOT-PSS nanowires, macroscopic silver conductive pads (4 mm long) were fabricated on the $\mathrm{SiO}_{2}$ substrates, which contact about 1000 PEDOT-PSS nanowires, while the distance between the pads was $2 \mathrm{~mm}$ (Figure 7).

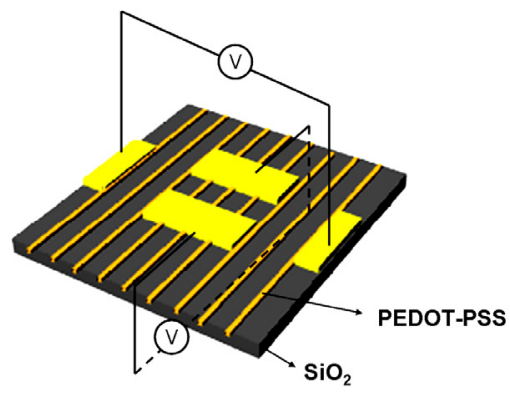

Figure 7. Cartoon showing the conductivity measurements of the PEDOT-PSS nanowires.

Conductivity measurements were carried out by a two-probe method at room temperature. Figure 8 shows the I-V characteristics of the PEDOT-PSS nanowires. Ohmic behavior was obtained at applied voltages from -1 to $1 \mathrm{~V}$ and the measured resistance from the I-V values (Figure 8, squares) was 3.6 M 2 . As a control experiment, we also measured the resistance perpendicular to the PEDOT-PSS nanowires, and no conductivity was 
recorded with this setup (Figure 8, circles), which indicates that there is no residual conductive polymer between the PEDOT-PSS nanowires and that the substrate is not contributing to the conductivity.

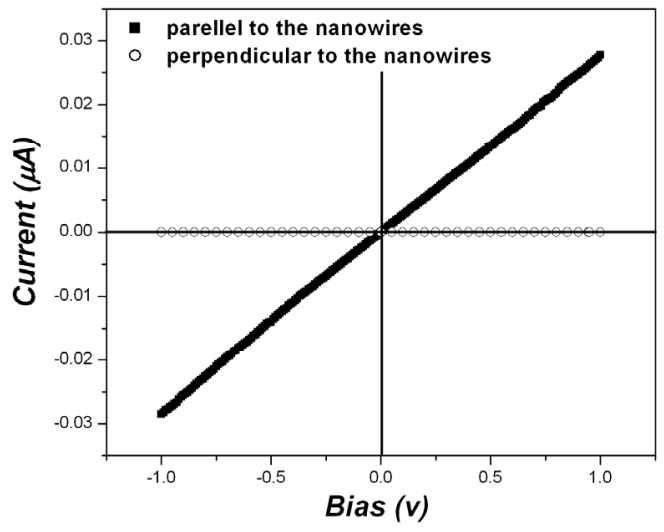

Figure 8. I-V conductivity measurement of the PEDOT nanowires fabricated by NAMIC using a $100 \times 110 \mathrm{~nm}$ mold.

By assuming that the material in each nanowire has the same conductivity as the bulk conductivity of PEDOT-PSS $(\sigma=1 \mathrm{~S} / \mathrm{cm})$, and incorporating the dimensions of the wires (height, $\mathrm{h}=100 \mathrm{~nm}$, width, $\mathrm{w}=100 \mathrm{~nm}$, length, $\mathrm{l}=2 \mathrm{~mm}$ ) the calculated overall resistance for about $\mathrm{n}=1000$ parallel contacted nanowires is $(1 / \mathrm{n})(1 / \sigma)(1 / \mathrm{wh})=2 \mathrm{M} \Omega$. This is quite close to the measured value $(3.6 \mathrm{M} \Omega)$, which indicates the good quality of the PEDOT-PSS nanowires as prepared by this method, and it proves that NAMIC has the potential to fabricate nano(opto)electronic or sensing devices.

\subsection{Conclusions}

In summary, a high-resolution soft lithographic technique - nanomolding in capillaries (NAMIC) - has been developed by using a composite PDMS stamp with sub-100 nm features fabricated by nanoimprint lithography. NAMIC has been used to pattern different functional materials from their solutions such as fluorescent dyes, proteins, nanoparticles, 
thermoplastic polymers, and conductive polymers at the nanometer scale. The resolution is limited only by the mold used in the NIL step. It is emphasized that NAMIC is a simple, versatile, and large-area patterning tool. The ability to pattern, in parallel, over several $\mathrm{cm}^{2}$ allows well-defined nanofeatures to be fabricated for high-throughput applications. Moreover NAMIC uses only simple materials (PDMS) and a low-cost available lithography technique (nanoimprint lithography) and thus provides access to nanopatterned structures at a substantially reduced cost, both in time and in equipment resources. It is believed that NAMIC will enable scientific opportunities to study the nano-confined self-assembly of different materials within the nanofluidic channels, and to apply it as a tool to fabricate nano(opto)electronic or sensing devices. For further development, it will be combined with different surface chemistries to achieve monolayer surface patterning at nanometer scales.

\subsection{Experimental}

\section{Materials and methods}

Imprint resist mr-I T85 and thermoplastic polymer mr-7010 were obtained from Micro Resist. Fluorescein (FITC)-conjugated ChromPure Human IgG, Fc Fragment was purchased from Jackon ImmunoResearch. The human IgGs had average 2.5 fluoresceinisothiocyanate labels per molecule and were dissolved at a concentration of $1 \mathrm{mg} / \mathrm{ml}$ in buffer solutions (0.01 M sodium phosphate, $0.25 \mathrm{M} \mathrm{NaCl}, \mathrm{pH}$ 7.6). The conducting polymer, PEDOT-PSS, was obtained as an aqueous suspension ( $\sim 1.3 \mathrm{wt} \%)$ from SigmaAldrich, and has a conductivity of $1 \mathrm{~S} / \mathrm{cm}$. All materials were used as received without further purification. A kit of PDMS prepolymer (Sylgard 184 silicon elastomer) and PDMS curing agent was purchased from Dow Corning Corporation. The $60 \mathrm{~nm} \mathrm{SiO} 2$ nanoparticles were synthesized according to a reported procedure. ${ }^{[28]}$

\section{Composite flat PDMS substrates}

Composite flat PDMS substrates were prepared by first casting the liquid prepolymer of the elastomer onto a polished Si wafer onto which an anti-adhesion layer had been deposited. After degassing, a piece of cover glass (MENZEL-GLÄSER BB015015A1 15 $\mathrm{mm} \times 15 \mathrm{~mm}$ ) which has been cleaned by piranha solution (3:1 mixture of sulfuric acid and hydrogen peroxide. CAUTION! Piranha solutions should be handled with great care in 
open containers in a fume hood. Piranha is highly corrosive and toxic and potentially explosive) was then put into the uncured mixture. The cover glass sank to the bottom of the PDMS liquid leaving a thin layer between the cover glass and the Si wafer. After curing at $60{ }^{\circ} \mathrm{C}$ for $8 \mathrm{hr}$ the stamp was peeled off from the Si wafer.

\section{NIL procedure}

Before spin coating the resist, the composite PDMS substrate was treated by short $\mathrm{O}_{2}$ plasma (10 mTorr; $10 \mathrm{~W} ; 20 \mathrm{sccm} \mathrm{O}_{2} ; 15 \mathrm{~s}$ ). This oxidation step increases the surface energy of the PDMS substrate to achieve a better film quality. A thin film of mr-I T85 solution was spin-coated onto the substrates, followed by a soft baking step for $2 \mathrm{~min}$ at $140{ }^{\circ} \mathrm{C}$ on a hot plate.

The imprint templates were made by edge lithography followed by wet etching. ${ }^{[17]}$ $1 \mathrm{H}, 1 \mathrm{H}, 2 \mathrm{H}, 2 \mathrm{H}$-Perfluorodecyltrichlorosilane was used as an anti-adherent layer to facilitate the stamp-imprint separation.

A stack of a composite PDMS substrate coated with mr-I T85 and a Si template was inserted into a hydraulic press (Specac), and the temperature was raised to $140{ }^{\circ} \mathrm{C}$. Then, 2 bar of pressure was applied to the system for $5 \mathrm{~min}$. Upon cooling to $50{ }^{\circ} \mathrm{C}$, the template was separated from the PDMS substrate.

\section{NAMIC procedure}

Before NAMIC, a short oxygen plasma step (10 mTorr; $10 \mathrm{~W} ; 20 \mathrm{sccm} \mathrm{O}_{2} ; 30 \mathrm{~s}$ ) was applied to the mold surface to promote adhesion upon contacting the substrate. Then, the mold was placed in contact with a substrate, which is cleaned by a piranha solution $(3: 1$ mixture of sulfuric acid and hydrogen peroxide. CAUTION! Piranha solutions should be handled with great care in open containers in a fume hood. Piranha is highly corrosive and toxic and potentially explosive.), so that the grooves form channels (capillaries). For good sealing, a few drops of water were applied immediately at the edges of the nanochannels. The water filling the channels generates a negative pressure and thus assists the sealing.

After water evaporation, a solution (typically $20 \mu \mathrm{l}$ ) was poured at the open end of the nanomold; the liquid spontaneously filled the channels under the effect of capillary pressure. Due to the high capillary pressure at the nanometer scale and the low viscosity of the solutions, the capillary filling time over large areas was relatively fast (seconds). The 
Large-area Nanoscale Patterning of Functional Materials by Nanomolding in Capillaries sample was then left for several hours, for the solutions to dry out. After the complete evaporation of the solvent, the mold was gently removed leaving the patterns on the surface.

\section{Contact pad fabrication for conductivity measurement}

A $300 \mathrm{~nm}$ thick $\mathrm{SiO}_{2}$ layer was formed on a silicon substrate by thermal growth as an insulating layer. PEDOT-PSS nanowires were fabricated by NAMIC on the substrates. Commercial available two-component silver pastes (SPI Conductive Silver Paste Plus) were used to fabricate the contact pads. After mixing the two components, the silver paste was carefully put onto the patterned PEDOT-PSS nanowires substrate and it was allowed to evaporate the solvent at $60{ }^{\circ} \mathrm{C}$ for $3 \mathrm{~h}$. In a typical setup, macroscopic silver contact pads (4 mm long) were fabricated on the $\mathrm{SiO}_{2}$ substrate orthogonal or parallel to the nanowires, which contact in the former case about 1000 PEDOT-PSS nanowires (Figure 7).

\section{Measurements}

AFM analyses were carried out with a NanoScope w (Veeco/Digital Instruments, Santa Barbara, CA, USA) Multimode Atomic Force Microscope equipped with a J-scanner, in tapping mode by using $\mathrm{Si}_{3} \mathrm{~N}_{4}$ cantilevers (Nanoprobes, Veeco/Digital Instruments) with a nominal spring constant of about $0.32 \mathrm{Nm}^{-1}$. AFM imaging was performed at ambient conditions.

Low-resolution SEM imaging was carried out with a JEOL, JSM-5610 SEM operating in secondary electron detection mode. High- resolution SEM imaging was carried out with a JEOL Gemini 1550 FEG-SEM.

Fluorescence Microscopy was performed using an Olympus inverted research microscope IX71 equipped with a mercury burner U-RFL-T as light source and a digital camera Olympus DP70 (12.5 million-pixel cooled digital color camera) for image acquisition. Red emission light $(\mathrm{A}>590 \mathrm{~nm})$ was filtered using a U-MWG Olympus filter cube.

Electrical measurements were carried out in a Suss MicroTec PM300 manual probe station with a standard Keithley 4200 semiconductor characterization system.

\subsection{References}


[1] E. Menard, M. A. Meitl, Y. G. Sun, J. U. Park, D. J. L. Shir, Y. S. Nam, S. Jeon, J. A. Rogers, Chem. Rev. 2007, 107, 1117.

[2] S. S. Shankar, L. Rizzello, R. Cingolani, R. Rinaldi, P. P. Pompa, ACS Nano 2009, 3,893 .

[3] M. C. McAlpine, R. S. Friedman, C. M. Lieber, Proc. IEEE 2005, 93, 1357.

[4] I. L. Medintz, H. T. Uyeda, E. R. Goldman, H. Mattoussi, Nature Mater. 2005, 4, 435.

[5] Y. N. Xia, G. M. Whitesides, Angew. Chem. Int. Ed. 1998, 37, 551.

[6] Y. N. Xia, J. A. Rogers, K. E. Paul, G. M. Whitesides, Chem. Rev. 1999, 99, 1823.

[7] E. Kim, Y. N. Xia, G. M. Whitesides, Nature 1995, 376, 581.

[8] M. Cavallini, C. Albonetti, F. Biscarini, Adv. Mater. 2009, 21, 1043.

[9] H. Schmid, B. Michel, Macromolecules 2000, 33, 3042.

[10] B. Michel, A. Bernard, A. Bietsch, E. Delamarche, M. Geissler, D. Juncker, H. Kind, J. P. Renault, H. Rothuizen, H. Schmid, P. Schmidt-Winkel, R. Stutz, H. Wolf, IBM. J. Res. Dev. 2001, 45, 697.

[11] W. S. Beh, I. T. Kim, D. Qin, Y. N. Xia, G. M. Whitesides, Adv. Mater. 1999, 11, 1038 .

[12] F. L. Zhang, T. Nyberg, O. Inganas, Nano Lett. 2002, 2, 1373.

[13] P. Greco, M. Cavallini, P. Stoliar, S. D. Quiroga, S. Dutta, S. Zachini, M. C. Lapalucci, V. Morandi, S. Milita, P. G. Merli, F. Biscarini, J. Am. Chem. Soc. 2008, 130, 1177.

[14] M. Cavallini, E. Bystrenova, M. Timko, M. Koneracka, V. Zavisova, P. Kopcansky, J. Phys.-Condens. Matter 2008, 20.

[15] E. Bystrenova, M. Facchini, M. Cavallini, M. G. Cacace, F. Biscarini, Angew. Chem., Int. Ed. 2006, 45, 4779.

[16] X. X. Duan, Y. Zhao, A. Perl,; E. Berenschot, D. N. Reinhoudt, J. Huskens, Adv. Mater. 2009, 21, 2798.

[17] Y. Zhao, E. Berenschot, M. de Boer, H. Jansen, N. Tas, J. Huskens, M. Elwenspoek, J. Micromech. Microeng. 2008, 18. 
Large-area Nanoscale Patterning of Functional Materials by Nanomolding in Capillaries

[18] A. V. Larsen, L. Poulsen, H. Birgens, M. Dufva, A. Kristensen, Lab Chip 2008, 8, 818.

[19] D. Nilsson, S. Balslev, A. Kristensen, J. Micromech. Microeng. 2005, 15, 296.

[20] P. Abgrall, N. T. Nguyen, Anal. Chem. 2008, 80, 2326.

[21] S. K. Arya, P. R. Solanki, M. Datta, B. D. Malhotra, Biosens. Bioelectron. 2009, 24,2810 .

[22] Y. Z. Dong, C. Shannon, Anal. Chem. 2000, 72, 2371.

[23] B. A. Parviz, D. Ryan, G. M. Whitesides, IEEE Trans. Adv. Packag. 2003, 26, 233.

[24] E. Kim, Y. N. Xia, G. M. Whitesides, J. Am. Chem. Soc. 1996, 118, 5722.

[25] S. Admassie, O. Inganas, J. Electrochem. Soc. 2004, 151, H153.

[26] M. Massi, C. Albonetti, M. Facchini, M. Cavallini, F. Biscarini, Adv. Mater. 2006, 18, 2739.

[27] G. Zotti, S. Zecchin, B. Vercelli, A. Berlin, S. Grimoldi, L. Groenendaal, R. Bertoncello, M. Natali, Chem. Mater. 2005, 17, 3681

[28] V. Mahalingam, S. Onclin, M. Peter, B. J. Ravoo, J. Huskens, D. N. Reinhoudt, Langmuir 2004, 20, 11756-11762. 



\section{Chapter 7}

\section{Assembly, Chemical Modification, and}

\section{Electrical Characterization of Quasi-1D}

\section{Gold Nanoparticle Arrays via}

\section{Nanomolding in Capillaries Combined}

\section{with Dithiocarbamate Bond Formation}

In this chapter, a high-resolution soft lithography technique - nanomolding in capillaries (NAMIC) - combined with dithiocarbamate (DTC) chemistry is used to fabricate sub-50 nm quasi-1D arrays of ultrasmall gold NPs over large areas. Owing to the chemical immobilization via the DTC bond, the patterned NPs systems are stable in water and organic solvents, thus allowing the surface modification of the patterned Au NP arrays through thiol chemistry and further orthogonal binding of proteins. The electrical properties of these patterned quasi-1D gold NPs arrays have also been studied. Our results show that NAMIC combined with surface chemistry is a simple but powerful tool to create metal nanoparticle arrays and potentially can be applied as a valuable tool to fabricate nano-electronic or bio-sensing devices. 


\subsection{Introduction}

Nanoparticles (NPs) have been the subject of intensive study because of their unique electronic, photonic, and magnetic properties, which make them potential candidates as elemental building blocks for the next generation of nanodevices. ${ }^{[1-5]}$ One of the keys to use the nanoparticles in future electronic technology is their controllable and precise assembly into ordered low-dimensional structures. Compared to randomly distributed particle arrays, ordered particle structures are better candidates for most practical applications. During the past few years, the demonstration of spatial ordering and the electronic properties of twoand three- dimensional NPs arrays have extensively been studied. ${ }^{[6-9]}$ As compared with two- or three-dimensional NPs arrays, NPs in one dimensional (1D) or quasi one dimensional arrays are of particular interest, both theoretically and experimentally, ${ }^{[10-16]}$ because of their value in understanding fundamental problems such as localization, hopping, and the formation of the energy bands in discrete but coupled systems. However, assembling 1D NPs arrays is much more challenging due to the difficulty in obtaining a long distance, continuously connected 1D superstructure. To circumvent the difficulty in constructing 1D structure from isotropic NPs, most previous studies have focused on assembling NPs on 1D templates such as DNA, $\left.{ }^{[17,} 18\right]$ nanowires, ${ }^{[19]} 1 \mathrm{D}$ structured substrate, ${ }^{[20,21]}$ templates fabricated by advanced lithography, ${ }^{[11,22-24]}$ etc. Although template assembly has brought a lot of impressive results, it is still desirable to develop new patterning methods of 1D NPs arrays directly on planar substrates for more facile production in large quantity and low cost with high-throughput.

In this chapter, a soft lithographical approach, nanomolding in capillaries (NAMIC) (see Chapter 6), combined with dithiocarbamate (DTC)-based surface chemistry ${ }^{[25,26]}$ was demonstrated to pattern ultra small gold nanoparticles (sub-2 $\mathrm{nm}$, core diameter) directly from their solutions into 1D NPs arrays on planar amine-terminated monolayer substrates over large areas. It uses a hybrid stamp with harder, more well-defined features, which was fabricated by nanoimprint lithography (NIL) on flat PDMS as the nanomold, thus allowing large areas (over several $\mathrm{cm}^{2}$ ) patterning of NPs arrays in one step, with relatively low cost. The covalent link of Au NPs on the substrate through DTC bond formation provides arrays 
having a uniform layer of NPs on the surface with high stability to organic solvents and water. These NP arrays can be used as model systems for studying the electrical properties of the 1D NP arrays. Moreover, due to the robust chemical immobilization, the patterned $\mathrm{Au}$ NPs can be further modified through functional thiols to bind proteins which potentially can be used for bio-sensing applications.

\subsection{Results and discussion}

\subsubsection{Fabrication scheme}

Scheme 1 shows a schematic outline of the NAMIC process combined with DTC chemistry to pattern Au NPs.

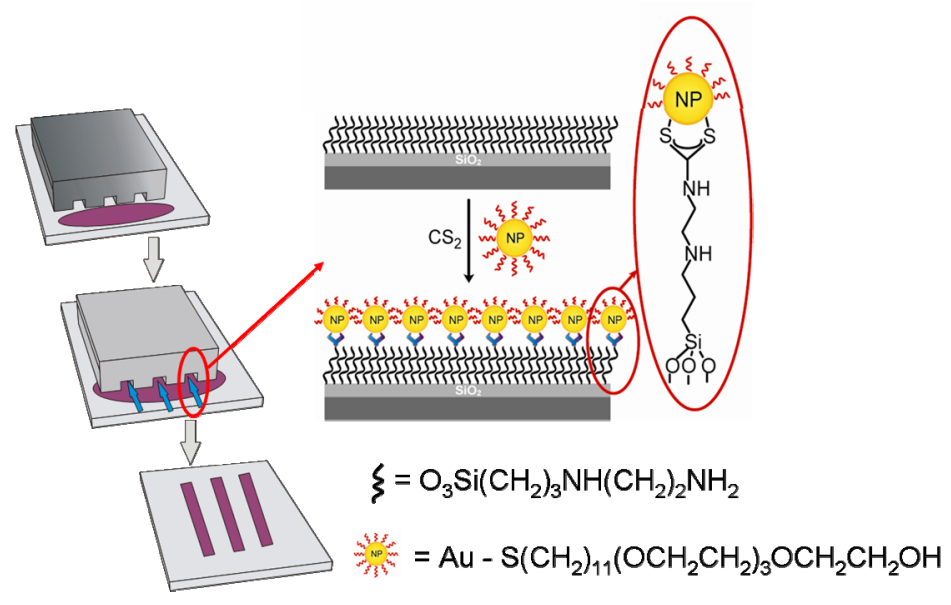

Scheme 1. Process scheme for nanomolding in capillaries (NAMIC) combined with dithiocarbamate (DTC) bond formation to pattern Au NPs into $1 D$ arrays.

First, an amine-functionalized SAM of $N$-[3-(trimethoxysilyl)propyl]ethylenediamine (TPEDA) was prepared on a silicon oxide surface. The hybrid PDMS nanomold was fabricated by nanoimprint lithography (NIL) with a thin film of thermal resist on a composite flat PDMS substrate. Then, the PDMS nanomold was soft bonded to the aminefunctionalized $\mathrm{SiO}_{2}$ substrates to form a parallel array of nanochannels (see also Chapter 6). 
The channels were then filled with a solution containing $\mathrm{CS}_{2}$ and Au-TOH NPs $(\sim 2 \mathrm{~nm}$ core diameter, coated with TOH (monohydroxy(1-mercaptoundecyl)tetraethylene glycol) ligands. Driven by capillary forces, the $\mathrm{CS}_{2}$ and $\mathrm{Au}-\mathrm{TOH}$ NPs solution fills the nanochannels. The primary amine on the surface is converted to DTC by reaction with $\mathrm{CS}_{2}$, which then displaces some TOH ligands on the Au NPs, effectively binding the Au NPs onto the surface within the confinement of the nanochannels. The process is simple, clean, and reliable and is based on water/alcohol solutions, providing eco-friendly fabrication. After evaporation of the solvent and detachment of the mold, the Au NPs are deposited on the substrate. The physisorbed NPs can be rinsed away by solvent, thus a uniform array of 1D Au NP lines is fabricated on the substrate.

\subsubsection{Quasi-1D nanoparticle arrays}

To fabricate the PDMS nanomold (see also Chapter 4), silicon templates with two different sizes of nanoridges (30 or $100 \mathrm{~nm}$ wide, $100 \mathrm{~nm}$ high, separated by $4 \mu \mathrm{m}$ ) were used as imprint molds. ${ }^{[27]}$ Thermal resist mr-I T85 (Micro Resist) was used at the imprint resist. The imprinting was performed under mild conditions $\left(150^{\circ} \mathrm{C}\right.$ and 2 bar). Polymer nanogrooves of 30 or $100 \pm 5 \mathrm{~nm}$ separated by $4 \mu \mathrm{m}$ were formed on the PDMS surface. A typical length of the PDMS nanomold was 0.5 to $1.5 \mathrm{~cm}$, and the width of the mold was 0.5 to $2 \mathrm{~cm}$, which can contain about 1250 to 5000 parallel channels. The Au-TOH NPs were prepared according to a published procedure. ${ }^{[26]}$

Figure 1a shows a representative TEM image of the Au NPs used in the assembly step. Measured from the TEM image, the Au NPs have an average diameter of $\sim 7 \mathrm{~nm}$ (metal core + the ligands size).

After NAMIC using a solution containing a 1:1 (v:v) mixture of a $200 \mathrm{nM}$ aqueous solution of NPs at $\mathrm{pH}=9.5$ and a $100 \mathrm{mM} \mathrm{CS}_{2}$ solution in ethanol, the Au NP patterns were thoroughly rinsed with ethanol to remove the physisorbed NPs and the substrates were further cleaned by oxygen plasma. Figure 1b-e shows the SEM and AFM height images of the $\mathrm{Au}$ NP patterns which were fabricated through a $30 \times 100 \mathrm{~nm}(\mathrm{w} \times \mathrm{h})$ PDMS nanomold onto an amine-functionalized $\mathrm{Si} / \mathrm{SiO}_{2}$ substrate. 
a)

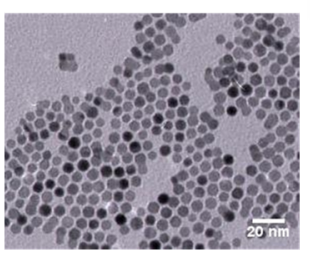

$60 \mathrm{~nm}$ b)

$60 \mathrm{~nm}$ b)

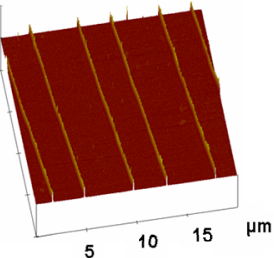

c)

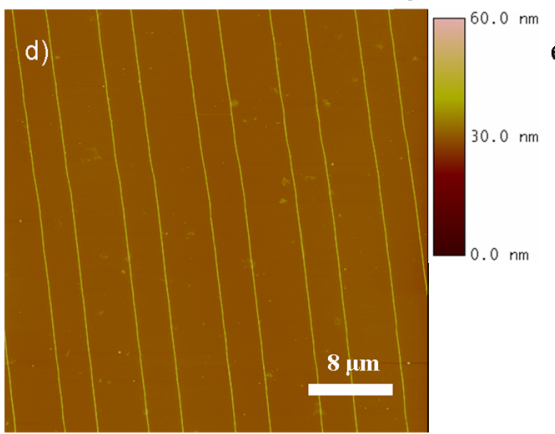

e)
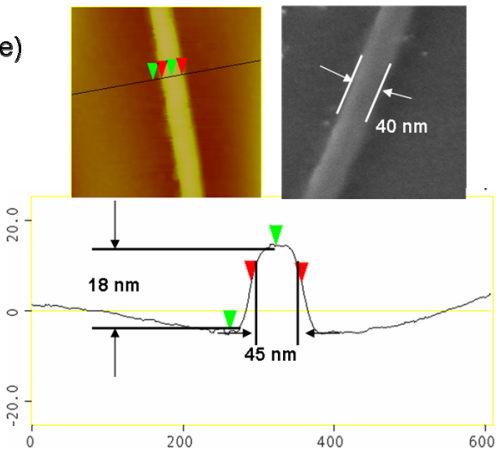

Figure 1. a) TEM image of the Au NPs used in the assembly step. 3D AFM (b), SEM (c), AFM height images (d) and section analysis (e) of Au NP patterns fabricated using a $30 \times$ $100 \mathrm{~nm}(w \times h)$ mold on $\mathrm{Si} / \mathrm{SiO}_{2}$ after $\mathrm{O}_{2}$ plasma by NAMIC combined with DTC chemistry.

As observed in the AFM and SEM images, after NAMIC and rinsing with solvent, the $\mathrm{Au}$ NPs formed continuous nanolines over large areas without obvious defects. The nanolines have an average width of $40 \pm 5 \mathrm{~nm}$, which is somewhat larger than the width of the PDMS nanomold $(30 \mathrm{~nm})$. This is probably due to the short oxygen plasma to the PDMS nanomold before the binding. The height of the Au NP arrays are $18 \pm 2 \mathrm{~nm}$ measured from AFM line scans. It did not change upon further sonication in ethanol. The height of the Au NP patterns $(\sim 18 \mathrm{~nm})$ corresponds to $\sim 2$ layers of the Au NPs, probably due to the closed packing of the Au NPs during the solvent evaporation within the nanochannels, while the width corresponds to about 5-6 NPs.

As a control experiment, we deposited Au-TOH NPs under the same conditions through NAMIC in the absence of $\mathrm{CS}_{2}$. After mold detachment and rinsing, we observed that the $\mathrm{Au}$ NPs formed broken lines on the substrates due to the rinsing with ethanol (Figure 2). Upon further sonication, the patterned Au NPs could be completely removed from the substrates. It proves that, in the absence of $\mathrm{CS}_{2}$, the Au-TOH NPs only physisorb on the substrates and that DTC bond formation is essential to achieve stable NP patterns. 


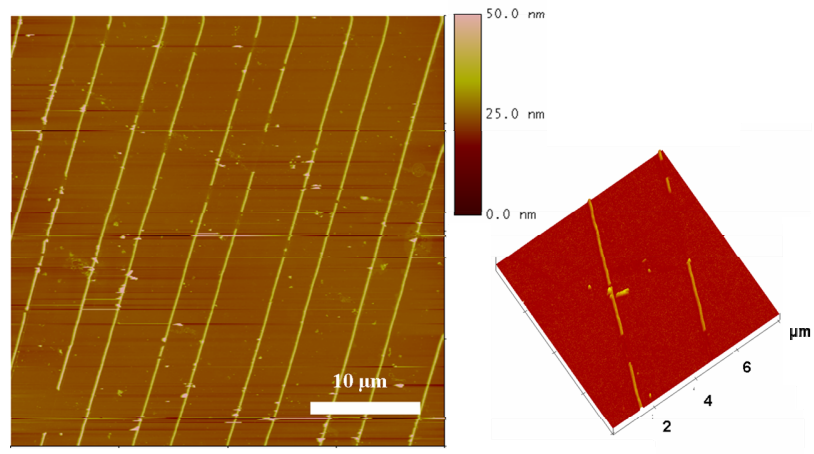

Figure 2. AFM images of the Au-TOH NPs deposited on $\mathrm{Si} / \mathrm{SiO}_{2}$ by $\mathrm{NAMIC}$ without $\mathrm{CS}_{2}$ added.

The NAMIC approach combined with DTC chemistry can be applied to different types of nanoparticles since $\mathrm{CS}_{2}$-mediated ligand exchange also works for NP systems featuring other thiophilic core materials. To demonstrate this versatility, we have patterned both coreshell CdSe-ZnS quantum dots (QD-TOH) and superparamagnetic FePt NPs (FePt-TOH) under the same conditions. Figure 3 shows a representative fluorescence micrograph and AFM image of both patterned NP arrays.
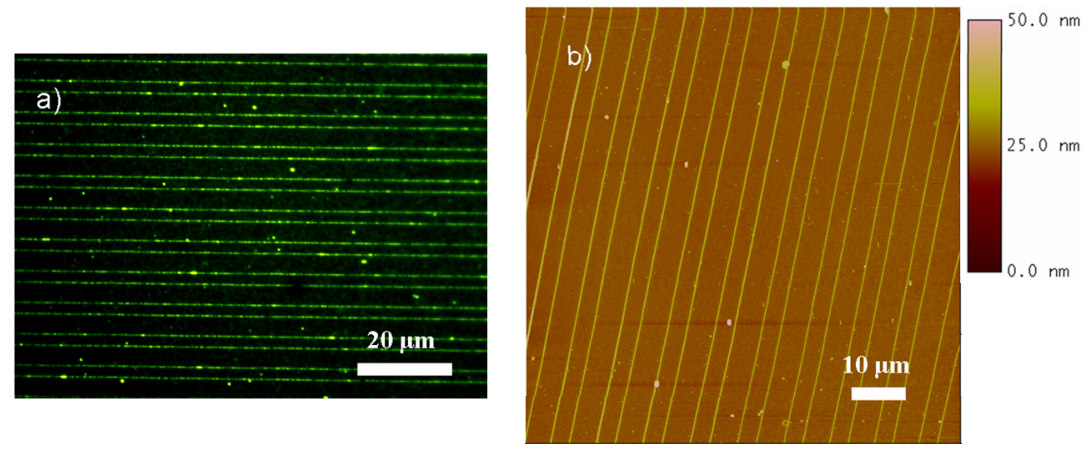

Figure 3. (a) Fluorescence micrograph of patterned QD-TOH NPs and (b) AFM height image of patterned FePt-TOH NPs on $\mathrm{Si} / \mathrm{SiO}_{2}$ by NAMIC combined with DTC chemistry using a $100 \times 100 \mathrm{~nm}(\mathrm{w} \times$ h) PDMS nanomold.

A substrate with prepatterned Au NP lines can be patterned by NAMIC again to create a second set of parallel lines to achieve cross patterning. Owing to the robust interactions 
by the DTC bond, the first Au NP lines are quite stable upon contacting with the second PDMS nanomold.

Figure $4 \mathrm{a}$ shows the process of making crossed lines by two sequential depositions. The first set of parallel Au NP lines was deposited on an amine-functionalized silicon substrate by NAMIC combined with DTC bond formation (Scheme 1). Upon PDMS demolding and rinsing with ethanol and further cleaning by $\mathrm{O}_{2}$ plasma, the TPEDA layer was regenerated from its solutions on the silicon dioxide surface. The substrate was then rotated by $90^{\circ}$ and contacted to another PDMS nanomold, and then Au-TOH NPs were deposited again under the same conditions. The second set of Au lines overlaps with the first one, forming a grid pattern (Figure $4 b-e)$.

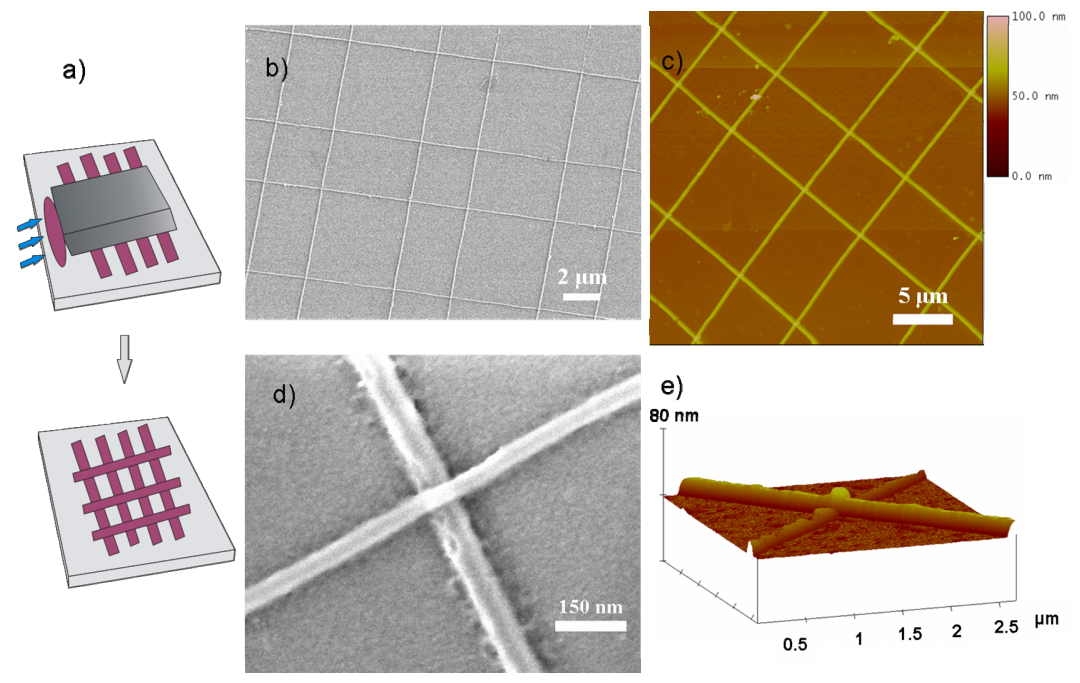

Figure 4. (a) Schematic diagram illustrating the grid patterns obtained by two sequential NAMIC steps. SEM (b) and AFM (c) images of Au-TOH NPs grid patterns fabricated on $\mathrm{Si} / \mathrm{SiO}_{2}$ by NAMIC combined with DTC chemistry using two times the $100 \times 100 \mathrm{~nm}(\mathrm{w} \times \mathrm{h})$ mold. SEM (d) and 3D AFM (e) images of the Au-TOH NP patterns by NAMIC combined with DTC chemistry using $1^{\text {st }}$ time the $30 \times 100 \mathrm{~nm}(\mathrm{w} \times \mathrm{h})$ and $2^{\text {nd }}$ time the $100 \times 100$ mold.

As clearly seen from the SEM and AFM images (Figure 4b-e), the Au NPs formed grid patterns over large areas. Nanolines overlapped one another with uniform width (100 or 40 $\pm 5 \mathrm{~nm}$ ) which compares well with the dimensions of the PDMS nanomold. The height of the lines is $18 \pm 2 \mathrm{~nm}$ except at the junctions $(\sim 30 \mathrm{~nm})$. This cross patterning through the 
NAMIC approach could provide a useful device platform for investigating the interactions between two different sets of NPs at the junctions of such grid patterns, if a second type of NPs is used.

\subsubsection{Au nanoparticle arrays as templates for assembly}

Owing to the stable chemical immobilization via the robust DTC bond, which is actually stronger than an Au-thiol bond, ${ }^{[25,26]}$ the patterned Au NP lines are rather stable in water and organic solvents, which allows further surface modification of the Au NPs through functionalized thiols for further applications.

As an example, a positively charged thiol ligand TTMA (HS- $\mathrm{C}_{11}$-tetra(ethylene glycol) $\mathrm{NMe}_{3}{ }^{+}$) was used to modify the surface of the Au nanolines by incubating the patterned Au nanolines in the TTMA ethanol solution $(0.1 \mu \mathrm{M})$ for $8 \mathrm{~h}$. The samples were then thoroughly washed with ethanol to achieve arrays of TTMA monolayer-functionalized Au nanolines (Au-TTMA). Due to the positive charges provided by the TTMA ligands, AuTTMA can be further used for the specific binding of negatively charged materials by electrostatic assembly (Figure 5a). Negatively charged, fluorescein isothiocyanate-labeled bovine serum albumin (FITC-BSA) was deposited onto the Au-TTMA from an aqueous solution. After rinsing with water, the samples were imaged by fluorescence microscopy and AFM (Figure 5b-e).

From the fluorescence images (Figure 5b,c), after surface modification of the Au NPs nanolines by TTMA and the post deposition of FITC-BSA, continuous fluorescence line patterns were observed over large areas on the substrate, thus indicating the successful deposition of FITC-BSA selectively on the positively charged Au-TTMA nanolines. Measuring from the AFM height images of the patterned Au nanolines before and after functionalization by TTMA and FITC-BSA (Figure 5d,e), the width and height of the nanolines have increased by $\sim 45 \mathrm{~nm}$ and $\sim 20 \mathrm{~nm}$ which both indicate a layer increase of $\sim 20 \mathrm{~nm}$. This shows a uniform attachment of FITC-BSA on Au nanolines via electrostatic interaction. 

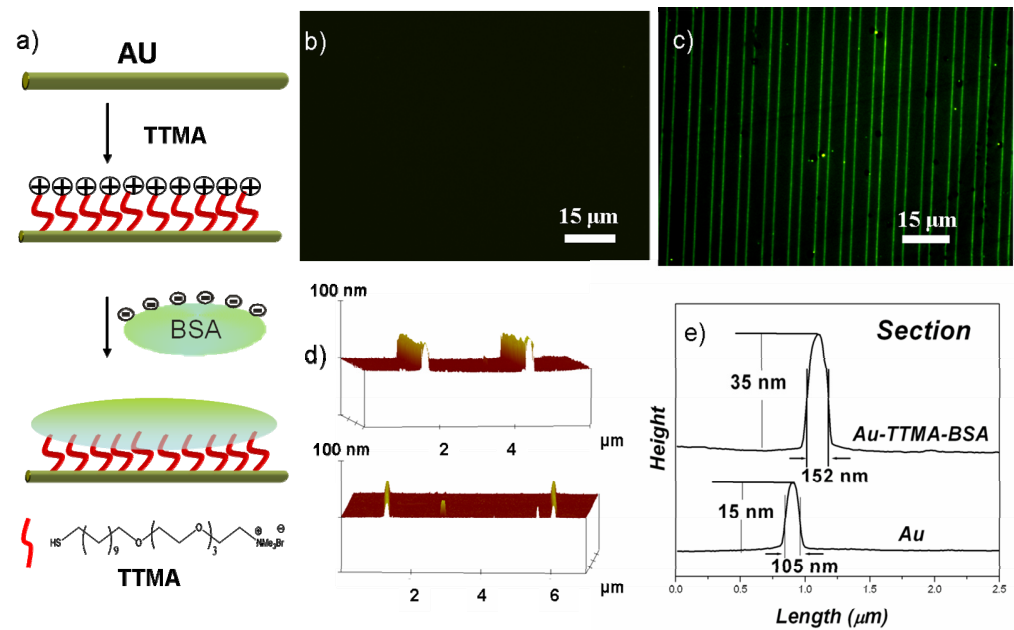

Figure 5. (a) Schematic illustration of the TTMA-functionalized Au nanolines and further deposition of fluorescently labeled BSA (FITC-BSA). Fluorescence micrographs $(b, c) A F M$ $3 D$ height images (d), and section analyses (e) of patterned Au nanolines before and after functionalization by the TTMA ligands and further electrostatic deposition of FITC-BSA.

\subsubsection{Electrical properties of the quasi-1D Au nanoparticles}

\section{arrays}

The electrical properties of monodisperse metal nanoparticles are interesting owing to their potential use as building blocks for nanoelectronic devices e.g. using the Coulomb blockade effect. $^{[28-30]}$ The Coulomb blockade normally happens at low temperatures for large particles. ${ }^{[31]}$ By decreasing the metal island size down to 1-2 nm, Coulomb blockade can be observed at room temperature which is important for real applications of single electron devices. ${ }^{[30]}$ However, to precisely position and integrate such small particles into functional devices is quite challenging. Previous efforts in this area have consisted primarily of lithographic patterning followed by random deposition ${ }^{[32,33]}$ or the electrostatic $^{[34]}$ or magnetic ${ }^{[35]}$ trapping of nanostructures. However, these approaches all suffer from some deficiency: they do not provide precise positioning, difficulty in obtaining long distance, large areas, continuous connection, require specific susceptibilities, have low throughput, and/or are not amenable to scaling. 
In this work, the NAMIC approach was used to pattern ultrasmall Au NPs. Within the nanochannels, the particles in the fluid were allowed to settle onto the substrate during the evaporation of the solvent. The flow and the self-organization of the particles offer a better chance to achieve ordered and continuous NPs patterns compared to other deposition methods. Besides, the formation of the DTC bond between the Au NPs and the substrate achieves specific binding which can provide systems having a stable and uniform layer of NPs on the surface. Furthermore, the large areas fabricated Au NPs arrays allow the possibility to study the electrical properties of multi-arrays of particles instead of only one or a few particles lines which reported before. ${ }^{[32-35]}$

In order to measure the electrical properties of the patterned $\mathrm{Au} N \mathrm{NP}$ arrays, macroscopic gold conductive pads were fabricated on the Au NP arrays on $\mathrm{SiO}_{2}$ substrates by thermal evaporation of gold and conventional lift-off procedures (Figure 6a). First, a 200 nm thick PMMA layer was spincoated onto the Au NP arrays, e-beam writing was then used to expose part of the PMMA at selected areas, and $2 \mathrm{~nm} \mathrm{Cr}$ was evaporated onto the substrate as the adhesion layer followed by $200 \mathrm{~nm}$ of gold. In the last step, the remaining PMMA was dissolved in acetone. The size of the fabricated gold contact pads was $80 \times 80$ $\mu \mathrm{m}$, which contacts about $20 \mathrm{Au}$ NPs lines, while the distance between two pads was 100 $\mu \mathrm{m}$ (Figure 6b,c).

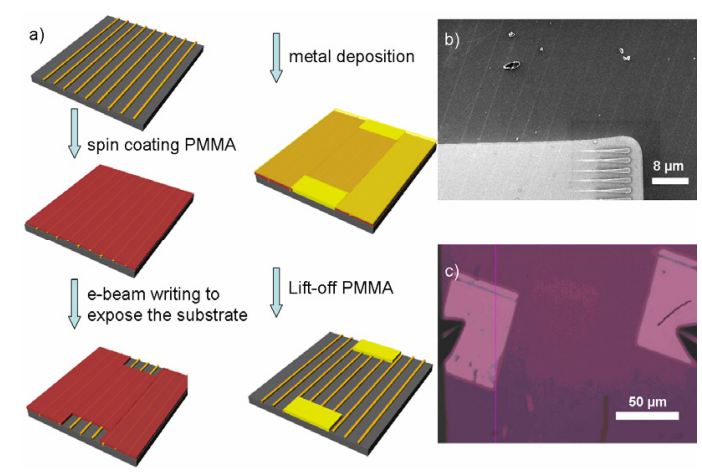

Figure 6. (a) Schematic illustration of the fabrication of the gold contact pads on Au NP arrays through a metal evaporation and lift-off process. SEM (b) and optical micrographs (c) of the fabricated gold contact pads. 
Conductivity measurements were carried out by a two-probe method at room temperature. Figure 7 shows the I-V characteristics of the Au NP arrays fabricated by NAMIC using a $30 \times 100 \mathrm{~nm}(\mathrm{w} \times \mathrm{h})$ mold on a $\mathrm{SiO}_{2}$ substrate. Non-ohmic current-voltage behavior was observed at applied voltages from -20 to $20 \mathrm{~V}$ (Figure $7 \mathrm{a}$ ). The curves are strongly nonlinear, nearly symmetrical at $\mathrm{V}=0$, and have non-zero threshold voltages.
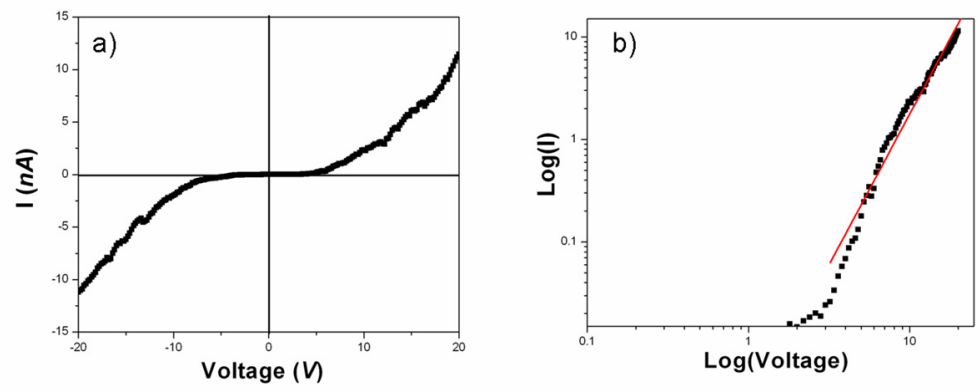

Figure 7. I-V characteristics (a) and the plot of log (I) versus log (V) (b) of the Au NPs arrays fabricated by NAMIC using a $30 \times 100 \mathrm{~nm}(w \times$ h) mold at room temperature.

The nanoparticles used in our study are composed of a gold core $(\sim 2 \mathrm{~nm})$ encapsulated by a monolayer shell of the $\mathrm{TOH}$ ligand. This core-shell structure provides an inter-core tunneling resistance, $R_{T}$, greater than the resistance quantum $h / e^{2}$ and should have a similar conducting behavior as in tunnel junction arrays. ${ }^{[36,37]}$ As studied by Middleton and Wingreen, ${ }^{[38]}$ electronic transport through an array of small metal particles separated by nanoscale gaps is determined by the interplay between the single-electron charging of an individual particle and the tunneling between adjacent particles. In the presence of a charge disorder due to quenched impurities in the insulating substrate under the array, this interplay leads to highly non-ohmic current-voltage (I/V) characteristics. They predicted current suppression below a threshold voltage $\left(V_{t}\right)$, while above this threshold the current follows a power law, $I \sim\left(V-V_{t}\right)^{\xi}$, with $\xi=1$ in $1 \mathrm{D}$ and between $5 / 3$ and 2 in $2 \mathrm{D}$.

In our case, as measured from SEM and AFM images (Figure 1d), the width and height of Au NPs patterns are 40 and $18 \mathrm{~nm}$, which corresponds to around 5-6 Au NPs in parallel and 2 layers high, which can be considered as quasi-1D Au NPs arrays. The I-V curve of the system should follow the power law which is predicted by Middleton and Wingreen. ${ }^{[38]}$ 
When the data are plotted as $\log (\mathrm{I})$ versus $\log (\mathrm{V})$ (Figure $7 \mathrm{~b}$ ), a nearly straight line is obtained. From the slope and the intercept of this line, we infer that $\xi \sim 2.9$ and $V_{t} \sim 2.7 \mathrm{~V}$, which are comparable to some published data for a single layer or a short $1 \mathrm{D}$ array of $\mathrm{Au}$ NPs with similar dimensions. ${ }^{[14,19,37,39]}$ This shows that our fabrication method represents a viable technique for creating large-area, continuous particle lines. Further developments may involve studying the electronic properties of these patterned quasi-1D nanoparticles arrays by changing the temperature or the gate voltage.

\subsection{Conclusions}

In summary, a high-resolution soft lithographic technique to pattern gold ( $\mathrm{Au})$ nanoparticles (NPs) on surface has been developed. Nanomolding in capillaries (NAMIC) has been combined with dithiocarbamate (DTC) chemistry to immobilize ultrasmall $\mathrm{Au}$ NPs from their solutions to form arrays of uniform and stable sub-50 nm quasi-1D Au NP nanolines over large areas. Combined with the DTC chemistry, NAMIC shows the possibilities to create stable NP patterns, both in water and organic solvents. The electrical properties of these patterned quasi-1D Au NP arrays have been studied and these are shown to be conducting and to have nonlinear I-V characteristics with non-zero threshold voltages consistent with Coulomb blockade behavior. The surface of the patterned Au NPs arrays can be further functioned by thiol ligands and bovine serum albumin (BSA) proteins were successfully adsorbed onto the Au NP arrays. Moreover, the technique is also effective for the patterning and immobilization of other thiophilic particles, including superparamagnetic CdSe-based QDs and FePt NPs. These results show that the NAMIC method combined with surface chemistry is a facile and powerful approach for the patterning of colloidal nanostructures over large areas and represent a significant step toward the integration of bottom-up chemically prepared nanostructures with lithographically patterned nanostructures. It is also believed that these techniques can be applied as a valuable tool to fabricate nano-electronic or bio-sensing devices.

\subsection{Experimental}


Assembling, Chemical Modification, and Electrical Addressing of Quasi-1D Au NP Arrays

\section{Materials and methods}

Imprint resist mr-I T85 was obtained from Micro Resist. The fluorescent isothiocyanate-labeled bovine serum albumin (FITC-BSA) and $N$-[3(trimethoxysilyl)propyl]ethylenediamine (TPEDA) were purchased from Aldrich. All materials were used as received without further purification. Au NPs, FePt NPs, CdSe based QDs, and TTMA (HS- $\mathrm{C}_{11}$-tetra(ethylene glycol) $\mathrm{NMe}_{3}{ }^{+}$) ligand were prepared in the lab of Prof. Vincent M. Rotello (UMASS at Amherst) according to published results. ${ }^{[26]}$

\section{Composite flat PDMS substrates}

Composite flat PDMS substrates were prepared by first casting the liquid prepolymer of the elastomer onto a polished Si wafer onto which an anti-adhesion layer had been deposited. After degassing, a piece of cover glass (MENZEL-GLÄSER BB015015A1 15 $\mathrm{mm} \times 15 \mathrm{~mm})$ which has been cleaned by piranha solution (3:1 mixture of sulfuric acid and hydrogen peroxide. CAUTION! Piranha solutions should be handled with great care in open containers in a fume hood. Piranha is highly corrosive and toxic and potentially explosive) was then put into the uncured mixture. The cover glass sank to the bottom of the PDMS liquid leaving a thin layer between the cover glass and the Si wafer. After curing at $60{ }^{\circ} \mathrm{C}$ for $8 \mathrm{~h}$ the stamp was peeled off from the Si wafer.

\section{NIL procedure}

Before spin coating the resist, the composite PDMS substrate was treated by a short $\mathrm{O}_{2}$ plasma step (10 mTorr; $10 \mathrm{~W} ; 20 \mathrm{sccm} \mathrm{O}$; $15 \mathrm{~s})$. This oxidation step increases the surface energy of the PDMS substrate to achieve a better film quality. A thin film of mr-I T85 solution was spin-coated onto the PDMS substrates, followed by a soft baking step for 2 $\min$ at $140{ }^{\circ} \mathrm{C}$ on a hot plate to form a $300 \mathrm{~nm}$ polymer layer.

The imprint templates were made by edge lithography followed by wet etching. ${ }^{[27]}$ $1 \mathrm{H}, 1 \mathrm{H}, 2 \mathrm{H}, 2 \mathrm{H}$-Perfluorodecyltrichlorosilane was used as an anti-adhesion layer to facilitate the stamp-imprint separation.

A stack of a composite PDMS substrate coated with mr-I T85 and a Si template was inserted into a hydraulic press (Specac), and the temperature was raised to $140{ }^{\circ} \mathrm{C}$. Then, 2 bar of pressure was applied to the system for $5 \mathrm{~min}$. Upon cooling to $50{ }^{\circ} \mathrm{C}$, the template was separated from the PDMS substrate. 


\section{Amine-terminated monolayer preparation}

Amine-terminated monolayers were prepared by immersing a cleaned silicon substrate into a $10 \mathrm{mM}$ solution of TPEDA in ethanol for $12 \mathrm{~h}$ under $\mathrm{N}_{2}$. The substrate was then removed from the solution and rinsed with $\mathrm{CH}_{2} \mathrm{Cl}_{2}$, ethanol, and $\mathrm{CH}_{2} \mathrm{Cl}_{2}$, followed by drying in a nitrogen flow.

\section{NAMIC procedure combined with dithiocarbamate (DTC) bond formation}

Before NAMIC, a short oxygen plasma step was used to pretreat the PDMS nanomold surface to promote adhesion upon contacting the substrate. Thereafter, the nanomold was placed on the amine-modified substrate, and an array of nanochannels was formed.

After the formation of the nanochannels, a drop of a NP solution containing 1:1 (v:v) mixture of a $200 \mathrm{nM}$ aqueous solution of Au NPs at $\mathrm{pH}=9.5$ and a $100 \mathrm{mM} \mathrm{CS}_{2}$ in ethanol solution was applied at the open ends of the nanochannels. Once the channels were filled completely, the solvent was allowed to evaporate at room temperature and the NPs precipitated out onto the substrate within the confinement of the channels. Once the solvent was completely evaporated, the PDMS nanomold was carefully peeled off from the substrate. Then the substrate was thoroughly rinsed with water and ethanol. Traces of residual T85 were observed on the substrate due to the oxygen plasma pretreatment of the nanomolds, but these could be easily removed by rinsing with an apolar solvent (e.g. cyclohexane, xylene) or by oxygen plasma.

\section{Post deposition of fluorescent BSA}

The patterned Au NPs arrays on silicon substrates were immersed in a TTMA in ethanol solution $(0.1 \mu \mathrm{M})$ for $8 \mathrm{~h}$. The samples were then thoroughly washed with ethanol and dried in a nitrogen stream to achieve arrays of TTMA-functionalized Au nanolines (Au-TTMA). Subsequently, the substrates were incubated with fluorescent FITC-BSA (1 $\mathrm{mg} / \mathrm{mL}$ ) in water for electrostatic assembly. After $1 \mathrm{~h}$, the substrates were washed with water several times and then dried in a nitrogen stream.

\section{Lithography for Au contact pads}

First, a $200 \mathrm{~nm}$ thick PMMA layer was spincoated onto the Au NP arrays, e-beam was then used to expose part of the PMMA at selected areas, and $2 \mathrm{~nm}$ of $\mathrm{Cr}$ was evaporated 
onto the substrate as the adhesion layer followed by $200 \mathrm{~nm}$ of gold. In the last step, the remaining PMMA was dissolved in acetone (Figure 6a).

\section{Measurements}

AFM analyses were carried out with a NanoScope $\amalg$ (Veeco/Digital Instruments, Santa Barbara, CA, USA) Multimode Atomic Force Microscope equipped with a J-scanner, in tapping mode by using $\mathrm{Si}_{3} \mathrm{~N}_{4}$ cantilevers (Nanoprobes, Veeco/Digital Instruments) with a nominal spring constant of about $0.32 \mathrm{Nm}^{-1}$. AFM imaging was performed at ambient conditions.

High- resolution SEM imaging was carried out with a JEOL Gemini 1550 FEG-SEM.

Fluorescence Microscopy was performed using an Olympus inverted research microscope IX71 equipped with a mercury burner U-RFL-T as light source and a digital camera Olympus DP70 (12.5 million-pixel cooled digital color camera) for image acquisition. Red emission light (A > $590 \mathrm{~nm}$ ) was filtered using a U-MWG Olympus filter cube.

Electrical measurements were carried out in a Suss MicroTec PM300 manual probe station with a standard Keithley 4200 semiconductor characterization system.

\section{Acknowledgements:}

Myoung-Hwan Park and Prof. Vincent M. Rotello are acknowledged for the collaboration and for facilitating a stay at UMASS (Amherst). Prof. Wilfred van der Wiel is thanked for helpful discussions.

\subsection{References}

[1] A. Eychmuller, J. Phys. Chem. B 2000, 104, 6514.

[2] R. Shenhar, T. B. Norsten, V. M. Rotello, Adv. Mater. 2005, 17, 657.

[3] G. Schmid, B. Corain, Eur. J. Inorg. Chem. 2003, 3081.

[4] S. K. Ghosh, T. Pal, Chem. Rev. 2007, 107, 4797.

[5] A. N. Shipway, E. Katz, I. Willner, ChemPhysChem 2000, 1, 18. 
[6] P. A. Kralchevsky, N. D. Denkov, V. N. Paunov, O. D. Velev, I. B. Ivanov, H. Yoshimura, K. Nagayama, J. Phys. Condens. Matter 1994, 6, A395.

[7] R. Parthasarathy, X. M. Lin, H. M. Jaeger, Phys. Rev. Lett. 2001, 87.

[8] N. Y. Morgan, C. A. Leatherdale, M. Drndic, M. V. Jarosz, M. A. Kastner, M. Bawendi, Phys. Rev. B 2002, 66.

[9] D. Yu, C. J. Wang, B. L. Wehrenberg, P. Guyot-Sionnest, Phys. Rev. lett. 2004, 92.

[10] J. Richardi, J. Chem. Phys. 2009, 130.

[11] K. Xu, L. D. Qin, J. R. Heath, Nat. Nanotechnol. 2009, 4, 368.

[12] Y. X. Zhang, H. C. Zeng, J. Phys. Chem. B 2006, 110, 16812.

[13] Z. Y. Tang, N. A. Kotov, Adv. Mater. 2005, 17, 951.

[14] K. Elteto, X. M. Lin, H. M. Jaeger, Phys. Rev. B 2005, 71.

[15] J. X. Huang, A. R. Tao, S. Connor, R. R. He, P. D. Yang, Nano Lett. 2006, 6, 524.

[16] N. V. Dziomkina, G. J. Vancso, Soft Matter 2005, 1, 265.

[17] C. M. Niemeyer, Angew. Chem. Int. Ed. 2001, 40, 4128.

[18] J. Richter, M. Mertig, W. Pompe, I. Monch, H. K. Schackert, Appl. Phys. Lett. 2001, 78, 536 .

[19] M. G. Ancona, S. E. Kooi, W. Kruppa, A. W. Snow, E. E. Foos, L. J. Whitman, D. Park, L. Shirey, Nano Lett. 2003, 3, 135.

[20] F. Westerlund, T. Bjornholm, Curr. Opin. Colloid Interface Sci. 2009, 14, 126.

[21] N. Kouklin, L. Menon, S. Bandyopadhyay, Appl. Phys. Lett. 2002, 80, 1649.

[22] Y. Cui, M. T. Bjork, J. A. Liddle, C. Sonnichsen, B. Boussert, A. P. Alivisatos, Nano Lett. 2004, 4, 1093.

[23] T. Kraus, L. Malaquin, H. Schmid, W. Riess, N. D. Spencer, H. Wolf, Nat. Nanotechnol. 2007, 2, 570.

[24] J. Y. Cheng, F. Zhang, V. P. Chuang, A. M. Mayes, C. A. Ross, Nano Lett. 2006, 6, 2099.

[25] M. H. Park, Y. Ofir, B. Samanta, V. M. Rotello, Adv. Mater. 2009, 21, 2323.

[26] M. H. Park, Y. Ofir, B. Samanta, P. Arumugam, O. R. Miranda, V. M. Rotello, Adv. Mater. 2008, 20, 4185. 
[27] Y. Zhao, E. Berenschot, M. de Boer, H. Jansen, N. Tas, J. Huskens, M. Elwenspoek, J. Micromech. Microeng. 2008, 18.

[28] A. N. Korotkov, R. H. Chen, K. K. Likharev, J. Appl. Phys. 1995, 78, 2520.

[29] D. L. Feldheim, K. C. Grabar, M. J. Natan, T. E. Mallouk, J. Am. Chem. Soc. 1996, 118,7640 .

[30] R. P. Andres, T. Bein, M. Dorogi, S. Feng, J. I. Henderson, C. P. Kubiak, W. Mahoney, R. G. Osifchin, R. Reifenberger, Science 1996, 272, 1323.

[31] T. Teranishi, C. R. Chim. 2003, 6, 979.

[32] D. L. Klein, R. Roth, A. K. L. Lim, A. P. Alivisatos, P. L. McEuen, Nature 1997, $389,699$.

[33] J. P. Spatz, V. Z. H. Chan, S. Mossmer, F. M. Kamm, A. Plettl, P. Ziemann, M. Moller, Adv. Mater. 2002, 14, 1827.

[34] A. Bezryadin, C. Dekker, G. Schmid, Appl. Phys. Lett. 1997, 71, 1273.

[35] C. S. Lee, H. Lee, R. M. Westervelt, Appl. Phys. Lett. 2001, 79, 3308.

[36] A. P. Alivisatos, K. P. Johnsson, X. G. Peng, T. E. Wilson, C. J. Loweth, M. P. Bruchez, P. G. Schultz, Nature 1996, 382, 609.

[37] L. Clarke, M. N. Wybourne, M. D. Yan, S. X. Cai, J. F. W. Keana, Appl. Phys. Lett. 1997, 71, 617.

[38] A. A. Middleton, N. S. Wingreen, Phys. Rev. Lett. 1993, 71, 3198.

[39] M. G. Ancona, W. Kruppa, R. W. Rendell, A. W. Snow, D. Park, J. B. Boos, Phys. Rev. B 2001, 64 . 



\section{Summary}

This thesis contributes to the continuous development of patterning strategies in several different areas of unconventional nanofabrication. A series of soft lithography approaches (microcontact printing, nanomolding in capillaries), nanoimprint lithography (NIL), and capillary force lithography (CFL) combined with different surface chemistry have been used to pattern or process different self-assembling systems (e.g. self-assembled monolayers, nanoparticles, (bio)molecules, and polymers) on surfaces. A focus is on high resolution and high materials versatility. Chapters 3-5 develop the concept of using chemically patterned flat PDMS to avoid the mechanical stability issue in conventional contact printing. With the combinations of NIL or CFL and different surface chemistry, high resolution chemical patterns have been fabricated on flat PDMS surfaces and these flat stamps can be used to transfer different thiol inks down to sub-100 nm. In Chapters 6 and 7, a hybrid PDMS nanomold was introduced and fabricated by thermal nanoimprint on a flat PDMS surface. The nanomold was used to pattern a large variety of materials (fluorescent dye, nanoparticles, (bio)molecules, polymers) through a wet lithography approach. The reached resolution and broad applicability of this approach demonstrate that the hybrid PDMS nanomold is a valuable tool for wet lithography patterning.

Chapter 1 provides a general introduction to this thesis.

Chapter 2 reviews recent achievements of patterning of self-assembling systems by soft lithography. Special attention was given to molding high-resolution features in elastomers that are mechanically stable, the need for specific surface chemistry or template design to adsorb molecules, nanoparticles, and biomolecules from solution onto selected areas of the stamp surface, and new strategies to transfer assemblies onto a target surface.

Chapter 3 introduces the concept of bifunctional, chemically patterned flat PDMS stamps to pattern hydrophilic ink molecules. Three different methods have been used to fabricate $1 \mathrm{H}, 1 \mathrm{H}, 2 \mathrm{H}, 2 \mathrm{H}$-perfluorodecyltrichlorosilane (PFDTS) and 3-(aminopropyl)triethoxysilane (APTS)-functionalized flat PDMS stamps. The method using two times 
oxygen plasma via full SAM formation, oxidation through a mask to simultaneously remove the first SAM locally and reactivate the PDMS, followed by attachment of the second silane has been proven to be the most efficient method. XPS, water contact angle, and AFM measurements have confirmed the chemical patterns on the PDMS surface. Attachment of a fluorescent dye to the amino-functionalized regions shows the possibility of further modification of the bifunctional stamps to achieve different chemical functionalities on the stamp. This new stamp design showed a good performance in microcontact printing $(\mu \mathrm{CP})$ of polar inks (G2-S dendrimer and TRITC dye) in both $(+) \mu \mathrm{CP}$ and (-) $\mu \mathrm{CP}$. Printing results of the G2-S dendrimer using the same stamps after one month confirmed the long-term stability of the chemically functionalized PDMS stamps.

Chapter 4 introduces a new method to create high resolution chemical patterns on flat PDMS surfaces. Thermal nanoimprint lithography (NIL) has been used to fabricate polymer patterns on flat PDMS at low pressure. The polymer patterns were used as a local mask to oxidize the flat PDMS substrate at the uncovered regions, in order to make chemically patterned flat stamps. Sub-100 nm gold lines were successfully replicated by these stamps through microcontact printing $(\mu \mathrm{CP})$. The resolution is limited only by the mold used in the imprint step, and by the wet etching step (when metal patterns are targeted). The combined NIL- $\mu$ CP procedure embodies advantages of NIL (high resolution) with those of $\mu \mathrm{CP}$, both regarding the flexible nature of PDMS (conformal contact in both the NIL and printing steps) and the flat stamp concept (high resolution, elimination of ink diffusion, and stamp deformation). It thus provides a versatile option to create highresolution patterns, especially when combined with the large chemical versatility of $\mu \mathrm{CP}$.

Chapter 5 shows a new nanopatterning strategy by the integration of capillary force lithography (CFL) and the flat stamp concept. High-resolution CFL was achieved by using patterned hard Si wafers as the mold and flat PDMS as the substrate. The formed polymer pattern was subsequently used as a mask for the chemical functionalization of the PDMS stamps. These flat stamps were mechanically stable and the chemical patterns on their surfaces allow one to control where ink transport takes place, on the sub-100 nm to many $\mu \mathrm{m}$ length scale. The use of flat PDMS as the substrate during CFL as well as the stamp in 
$\mu \mathrm{CP}$ increases the resolution of both methods. The combined CFL- $\mu \mathrm{CP}$ procedure provides a convenient and inexpensive method to create high-resolution patterns.

Chapter 6 presents a high-resolution soft lithography technique - nanomolding in capillaries (NAMIC). NAMIC has been developed by using a composite PDMS stamp with sub-100 nm features fabricated by nanoimprint lithography. NAMIC has been used to pattern different functional materials from their solutions such as fluorescent dyes, proteins, nanoparticles, thermoplastic polymers, and conductive polymers at the nanometer scale. The resolution is limited only by the mold used in the NIL step. It is emphasized that NAMIC is a simple, versatile, and large-area patterning tool. The ability to pattern, in parallel, over several $\mathrm{cm}^{2}$ allows well-defined nanofeatures to be fabricated for highthroughput applications. Moreover NAMIC only uses simple materials (PDMS) and a lowcost lithography technique (NIL) and thus provides access to nanopatterned structures at a substantially reduced cost, both in time and in equipment resources. We believe that NAMIC will enable scientific opportunities to study the nano-confined self-assembly of different materials within the nanofluidic channels, and to apply it as a tool to fabricate nano(opto)electronic or sensing devices.

Chapter 7 represents a high-resolution soft lithography technique to pattern gold $(\mathrm{Au})$ nanoparticles (NPs) on surfaces. Nanomolding in capillaries (NAMIC) has been combined with dithiocarbamate (DTC) chemistry to immobilize ultrasmall Au NPs from their solutions to form arrays of uniform and stable sub-50 nm quasi-1D Au nanolines over a large area $\left(>\mathrm{cm}^{2}\right)$. The electrical properties of these patterned quasi-1D Au NP arrays have been studied. They are shown to be conducting and to have nonlinear I-V characteristics with non-zero threshold voltages consistent with a Coulomb blockade behavior. Owing to the chemical immobilization through the robust DTC bond, the patterned NP systems are stable in water and organic solvents. Thus, the surface of the patterned Au NPs arrays can be further functioned by thiol ligands, and bovine serum albumin (BSA) was successfully adsorbed onto the Au NP arrays via orthogonal assembly. Moreover, the technique is also effective for the patterning and immobilization of other thiophilic particles, including super-paramagnetic CdSe-based QDs and FePt NPs. These results show that NAMIC combined with surface chemistry is a facile and powerful approach for the patterning of 
colloidal nanostructures over large areas and thus represents a significant step toward the integration of bottom-up chemically prepared nanostructures with lithographically patterned nanostructures.

Collectively, the results in this thesis illustrate some new unconventional nanofabrication approaches in the patterning of matter on the sub-100 nm scale. The formation of bifunctional, chemically patterned flat PDMS stamps improved the compatibility of the PDMS with polar inks by having hydrophilic patterns on the PDMS surface. The combination of nanoimprint lithography or capillary force lithography with flat stamp concept opens new ways to fabricate chemical patterns on flat PDMS and improves the printing resolution down to sub-100 $\mathrm{nm}$. The use of a hybrid PDMS nanomold as a template for a wet lithography approach has created a simple but powerful tool to pattern different kinds of material at the nanoscale. These high-resolution soft lithography approaches developed in this thesis are ready to be used in normal research labs as tools to pattern different molecules or nanomaterials to functional nanostructures especially, when combined with the large chemical versatility of soft lithography. I also believe these tools are valuable to fabricate future nano-electronic or bio-sensing devices. 


\section{Samenvatting}

Dit proefschrift levert een bijdrage aan de continue ontwikkeling van strategieën tot patroneren in meerdere gebieden van onconventionele nanofabricage. Een serie zachtlithografische methoden (microcontact-drukken, nanovormen in capillairen), nanoimprintlithografie (NIL) en capillaire-kracht-lithografie (capillary force lithography; CFL) gecombineerd met verschillende oppervlaktechemie is gebruikt voor het patroneren of bewerken van zelf-assemblerende systemen (bv. zelf-geassembleerde monolagen (selfassembled monolayers; SAMs), nanodeeltjes, (bio)moleculen en polymeren) op oppervlakken. De focus ligt op een hoge resolutie en een grote veelzijdigheid in materiaalkeuze. In hoofdstukken 3-5 wordt het concept van chemisch gepatroneerd vlak PDMS (polydimethylsiloxaan) geïntroduceerd, ter voorkoming van de mechanische stabiliteitsproblemen die optreden in traditioneel microcontact-drukken. Door de combinatie van NIL of CFL met verschillende oppervlaktechemie, zijn hoge-resolutie chemische patronen verkregen op vlakke PDMS-oppervlakken, en deze vlakke stempels zijn gebruikt voor het overbrengen van verschillende thiol-inkten tot op sub-100-nm-schaal. In hoofdstukken 6 en 7 wordt een hybride PDMS-nanomal geïntroduceerd, gefabriceerd door thermische NIL op een vlak PDMS-oppervlak. De nanomal is gebruikt voor het patroneren van een reeks materialen (fluorescerende kleurstoffen, nanodeeltjes, (bio)moleculen, polymeren) met een nat-lithografische benadering. De behaalde resolutie en brede toepasbaarheid van deze benadering demonstreert dat de hybride PDMS-nanomal een waardevolle methode is voor nat-lithografisch patroneren.

Hoofdstuk 1 geeft een algemene inleiding tot dit proefschrift.

Hoofdstuk 2 behandelt recente resultaten op het gebied van patroneren van zelfgeassembleerde systemen met zachte lithografie. Extra aandacht is gegeven aan het vormen van hoge-resolutie-kenmerken in mechanisch stabiele elastomeren, aan de behoefte voor specifieke oppervlakte-chemie of ontwerp van de afdruk voor het adsorberen van moleculen, nanodeeltjes en biomoleculen uit oplossing op geselecteerde delen van het 
stempeloppervlak en aan nieuwe strategieën voor het overbrengen van assemblages op het doeloppervlak.

Hoofdstuk 3 introduceert het concept van bifunctionele, chemisch gepatroneerde vlakke PDMS-stempels voor het patroneren van hydrofiele inktmoleculen. Drie verschillende methoden zijn gebruikt voor het maken van vlakke PDMS-stempels gefunctionaliseerd met $1 \mathrm{H}, 1 \mathrm{H}, 2 \mathrm{H}, 2 \mathrm{H}$-perfluorodecyltrichlorosilaan (PFDTS) en 3(aminopropyl)-triethoxysilaan (APTS). De methode die twee keer gebruik maakt van zuurstofplasma en volledige monolaag-vorming, oxidatie door een masker voor gelijktijdige lokale verwijdering van de eerste monolaag en activering van het PDMS gevolgd door aanhechting van de tweede silaan heeft zich als meest effectieve methode uitgewezen. XPS, contacthoek-goniometrie en AFM-metingen hebben de chemische patronen op het PDMS-oppervlak aangetoond. De binding van een fluorescerende kleurstof aan de amino-gefunctionaliseerde gebieden demonstreert de mogelijkheid tot verdere modificatie van de bifunctionele stempels met andere functionele groepen. Dit nieuwe stempelontwerp heeft zich bewezen in het microcontact-drukken $(\mu \mathrm{CP})$ van polaire inkten (G2-S-dendrimeer en TRITC-kleurstof) in (+) $\mu \mathrm{CP}$ en (-) $\mu \mathrm{CP}$. Afdrukken van het G2-Sdendrimeer, gebruik makend van dezelfde stempel na een maand, bevestigt de langetermijn-stabiliteit van de chemisch gepatroneerde PDMS-stempels.

In hoofdstuk 4 wordt een nieuwe methode voor het maken van hoge-resolutie chemische patronen op vlakke PDMS-oppervlakken geïntroduceerd. Thermische NIL is gebruikt voor het fabriceren van polymeerpatronen op vlak PDMS onder lage imprintdruk. Het polymeerpatroon is vervolgens gebruikt als lokaal masker om het PDMS op de niet bedekte delen te oxideren en op deze manier chemisch gepatroneerde vlakke stempels te produceren. Met behulp van $\mu \mathrm{CP}$ zijn met deze stempels sub-100-nm-goudlijntjes succesvol gerepliceerd. De resolutie is alleen gelimiteerd door de mal, gebruikt tijdens het imprinten, en de natchemische etsstap (als metaalpatronen het doel zijn). De gecombineerde NIL- $\mu$ CP-procedure verenigt de voordelen van NIL (hoge resolutie) met die van $\mu \mathrm{CP}$, gebruik makend van het flexibele karakter van PDMS ("conformal" contact in zowel de NIL- als de afdruk-stappen) en het vlakke-stempel-concept (hoge resolutie, eliminatie van inktdiffusie en stempelvervorming). Het biedt dus een veelzijdige manier om hoge- 
resolutie-patronen te maken, in het bijzonder in combinatie met de grote chemische veelzijdigheid van $\mu \mathrm{CP}$.

Hoofdstuk 5 beschrijft een nieuwe nanopatroneringsstrategie door de integratie van CFL met het vlakke-stempel-concept. Hoge-resolutie CFL is gedemonstreerd met behulp van gepatroneerde harde Si-wafers als mal en vlak PDMS als substraat. Het gevormde polymeerpatroon is vervolgens gebruikt als masker voor de chemische functionalisering van de PDMS-stempels. De mechanische stabiliteit en het chemische patroon op het oppervlak van deze vlakke stempels maakt de plaats van inktoverdracht controleerbaar, van sub-100 nm tot meerdere- $\mu \mathrm{m}$ lengteschaal. Het gebruik van vlak PDMS als substraat in CFL en als stempel in $\mu \mathrm{CP}$ verhoogt de resolutie van beide methoden. Het gecombineerde $\mathrm{CFL}-\mu \mathrm{CP}$-proces biedt een doelmatige en goedkope methode om hoge-resolutie patronen te vormen.

Hoofdstuk 6 presenteert een hoge-resolutie zacht-lithografische techniek: nanovormen in capillairen (NAMIC). NAMIC maakt gebruik van een composiet PDMS-stempel met sub-100-nm-structuren gefabriceerd met NIL. Met NAMIC zijn diverse functionele materialen zoals fluorescerende kleurstoffen, eiwitten, nanodeeltjes, thermoplastische polymeren en geleidende polymeren uit oplossing op nanometerschaal gepatroneerd. De resolutie is alleen gelimiteerd door de mal gebruikt tijdens de NIL-stap. Benadrukt wordt dat NAMIC een eenvoudige, veelzijdige en voor grote oppervlakken geschikte patroneringsmethode is. De mogelijkheid tot patroneren, parallel en over meerdere $\mathrm{cm}^{2}$, maakt de fabricage van goed gedefiniëerde nanostructuren met hoge doorvoercapaciteittoepassingen mogelijk. Bovendien maakt NAMIC alleen gebruik van eenvoudige materialen (PDMS) en voordelige lithografische technieken (NIL), toegang gevend tot nanogepatroneerde structuren voor substantieel gereduceerde kosten, zowel qua tijd en apparatuur. NAMIC biedt aldus de mogelijkheid tot wetenschappelijk onderzoek naar nanobegrensde zelf-assemblage van verschillende materialen in nanofluidische kanalen, en het is toepasbaar als methode voor de fabricage van nano(opto)elektronische- of sensorapparatuur.

Hoofdstuk 7 vermeldt een hoge-resolutie zacht-lithografische techniek om gouden ( $\mathrm{Au})$ nanodeeltjes (nanoparticles; NPs) op oppervlakken te patroneren. Nanovormen in 
capillairen (NAMIC) gecombineerd met dithiocarbamaat- (DTC-) chemie is gebruikt ter hechting van zeer kleine Au-NPs uit oplossing om pakketten gelijkmatige en stabiele sub$50 \mathrm{~nm}$ quasi-1D gouden nanolijnen over grote gebieden $\left(>\mathrm{cm}^{2}\right)$ te vormen. De elektrische eigenschappen van deze gepatroneerde quasi-1D Au-NP-pakketten is onderzocht. Geleiding vertoonde niet-lineaire I-V-kenmerken en een zekere drempelspanning in overeenstemming met Coulomb-blokkade. Door de chemische hechting via de robuuste DTC-binding zijn de gepatroneerde NP-systemen stabiel in water en in organische oplosmiddelen. Het oppervlak van de gepatroneerde NP-pakketten kan dus verder gefunctionaliseerd worden met thiolliganden en runder-serum-albumine (BSA) kon succesvol via orthogonale assemblage op de Au-NP-pakketten geadsorbeerd worden. De techniek is bovendien toepasbaar voor de patronering en hechting van andere thiofiele deeltjes, inclusief superparamagnetische CdSegebaseerde "quantum dots" en FePt-NPs. De resultaten tonen aan dat NAMIC in combinatie met oppervlaktechemie een snelle en krachtige methode is voor het patroneren van colloïdale nanostructuren over grote oppervlakken, die daarmee een significante stap zet in de richting van integratie van bottom-up chemisch bereide nanostructuren met lithografisch gepatroneerde nanostructuren.

Samenvattend illustreert dit proefschrift enkele nieuwe onconventionele nanofabricagebenaderingen voor het patroneren van materie op de sub-100-nm-schaal. De vorming van bifunctionele, chemisch gepatroneerde vlakke PDMS-stempels verbeterde de bruikbaarheid van PDMS voor polaire inkten door het maken van hydrofiele patronen op het PDMSoppervlak. De combinatie van NIL of CFL met het vlakke-stempel-concept opent nieuwe wegen voor de fabricage van chemische patronen op vlak PDMS en verbetert de afdrukresolutie tot sub-100 nm. Het gebruik van een hybride PDMS-nanomal als sjabloon voor een nat-lithografische benadering heeft een eenvoudige maar krachtige methode opgeleverd voor het patroneren van diverse soorten materialen op de nanoschaal. De in dit proefschrift ontwikkelde hoge-resolutie, zacht-lithografische benaderingen zijn klaar voor gebruik in normale onderzoekslaboratoria als methode voor de patronering van verscheidene moleculen of nanomaterialen tot functionele nanostructuren, in het bijzonder in combinatie met de grote chemische veelzijdigheid van zachte lithografie. Deze processen 
zullen waardevol zijn voor de fabricage van toekomstige nano-electronica of bio-analyseapparatuur. 



\section{Acknowledgement}

Finally, it comes to the end. The last remaining part is to acknowledge all those people that have contributed to the work described in this thesis. This is an impossible task, given the many people that have helped to design, implement, apply, criticize, and sponsor the work. I am going to try anyway, and if your name is not listed, rest assured that my gratitude is not less than for those listed below.

First and foremost I would like to express my deep and sincere gratitude to my promotor and daily supervisor Professor Jurriaan Huskens. Dear Jurriaan, it has been an honor to be your first PhD student after you promoted to full professor. I appreciate all your contribution of time, ideas, and funding to make my $\mathrm{PhD}$ experience productive and stimulating. You have taught me, both consciously and un-consciously, how a good scientist should act. Your joy and enthusiasm for research, conscientious attitude towards work, perseverance, creativity and good organization was contagious and motivational for me. You are also quite open-minded which gave me enough freedom to choose my research orientation. I also have to thank you for your encouragement and understanding during my tough times in the $\mathrm{PhD}$ pursuit.

I am deeply grateful to my promotor, Professor David Reinhoudt. Dear David, thanks a lot for your suggestions, fresh ideas, encouragement and support during my $\mathrm{PhD}$ and stay at Twente. There are so many things I could learn from you as a successful scientist.

Besides my advisors, I have to thank a few former and present staff members in SMCT and MNF. Maria Péter supervised me at beginning of my PhD. Thanks for all the corrections you made to my report and the AFM instructions. Many thanks to Bart Jan and Pascal, who gave me a lot of suggestions for my research. I am also grateful to Wim, thanks for proofreading my thesis and for the careful corrections you made. Richard, thanks for your patience and help with all the problems that I have met with the computers, networks, etc. It is always nice to listen to the stories from your holiday trips. Marcel, thanks for all of your assistance in the chemical labs. I am pretty sure that you are the tallest friend I have 
had in my life. I would like to thank Ben Lammerink for the help with all of the paper work at beginning of my $\mathrm{PhD}$, and the moving of my apartment. Our secretaries, Izabel, Gerardine, and ex-secretaries, Melissa, Danielle, Marieke are thanked for handling the administrative works.

I still remember when I came to the interview, I hardly knew anything about lithography and it was rather a big challenge for me to work on this project as I only had a chemistry background. Thanks to the open environment of MESA+, which gave me the chance to meet and collaborate with many smart scientists and researchers from different backgrounds. I really enjoyed working in this atmosphere, collaborating with chemists, physicists, biologists, and engineers. Without these collaborations and contributions from different groups, the thesis would not have been possible.

For the collaborations, I would like to give my first thanks to Yiping Zhao, Erwin Berenschot, Niels Tas, and Henri Jansen from the Transducers Science and Technology (TST) group. Yiping, I cannot remember how many times I have asked to you to make imprint molds for me but if you look through my thesis you will see the importance of your nanoridges. Without those molds, the thesis would not be possible. Thank you for your patience and clever hands. I also have to thank Erwin for the careful corrections to our papers and Niels for the suggestions and discussions.

The work described in Chapter 7 was done together with Myoung-Hwan Park from UMass (Amherst). This chapter would not have been possible without your contributions. Myoung, it was really nice to work together with you and thanks for your nanoparticles and kind help at UMass. I would like to thank Professor Vincent Rotello for offering me a chance to come to Amherst and for facilitating the stay at UMass. I also have to thank a few friends that I made during my stay there: Yuxi Mu, Zhengjiang Zhu, and Jiansheng Feng, thanks for all of your help which made my life at Amherst much easier and unforgettable.

I am also indebted to Professor Wilfred van der Wiel, the chair of the NanoElectronics group. Thanks for your helpful suggestions and discussions in regards to all the electrical measurements.

During the work of my thesis, I had to spend a lot of time at the MESA+ cleanroom. I would like to thank all the people from the cleanroom and in particular Rene for the help 
with the RIE setup and Huib for the imprinting. I also have to thank Mark for the high resolution SEM imaging, Gerard Kip for XPS measurements, and Sander for the Probe Station setup.

I gratefully acknowledge the financial support by NanoNed, a national nanotechnology program coordinated by the Dutch Ministry of Economic Affairs. I need to thank all the NanoNed staff and user committee members within the Nanofabrication Flagship. Many successful meetings have been organized, and I have benefited a lot from these meetings. Special thanks to Dr. Jos Joore for inviting me to Pepscan and for the collaborations.

I would like to thank all the group members who during my stay at Twente, have provided such a pleasant, friendly, and international environment, for learning from each other, sharing different cultures and enjoying some outings. My first thanks go to my paranimfen Pieter Moonen and Shuhan Hsu. Pieter, you are such a well-organized person with a little bit typical german stubbornness. Thanks for all the Dutch translations you have made for me, especially the thesis summary. I wish you all the best for the rest of your $\mathrm{PhD}$ and your future career. Shuhan, I wish I could be as positive as you can. I really enjoyed your clear and cute laugh which I could even hear from the other end of the corridor. Thanks for being there to listen to me and to encourage me when I met troubles. I wish that you will always be happy. Lanti, such a quiet and polite lady, thanks for proofreading my thesis, I hope your little baby also enjoyed her first nanotechnology instruction. $\left({ }^{\wedge} \wedge\right.$ ) Jordi, thanks for reading my thesis as well. You are always very nice and present in a cool fashion. Good luck and best wishes for your project. I also have to thank many other former and present lab mates for their kind help: Xuemei for all the help at beginning of my $\mathrm{PhD}$ time; Veera for all the discussions with the project and introduction to the surface chemistry; András for the dendrimer inks and contact printing introduction; Pascale for the imprint introduction; Christiaan for the kind help in the cleanroom and nice introduction to the photolithography tricks; Xingyi and Arántzazu for the preparation of the nanoparticles; Alberto for the AFM setups; Huaping for the nice discussions with all the problems I have met; Kim for my English proofs; Chien-Ching and Maryana for going with me to many conferences, meetings; Janet for sharing the measurement times; Sven and Pim for the electrical measurements; and the many others who have taught, helped and supported me: 
Manon, Sachin, Lourdes, Christian, Tieme, Aldrik, Bianca, Francesca, Oktay, Henk, Dae June, Elisabetta, Martine, Ignacio, Srinidhi, Deniz, Albert, Mirko, Dorota, Denis, Jealemy, Vijay, Raluca, Carlo etc.

During these years, many Chinese friends came to Twente and shared nice moments with me. It is hard to mention all your names but I would like to mention a few of them: Bai wei, Chen Songyue, and Xu lixian (my dear neighbors, thanks for all the dinners, talks, and the support) He yizhi, He tao, Xu jiang, Wu zhongkai, Song chunlin, Zhou weihua, Gu hao, Shao xiaoying (Thanks for all the help when needed); Xiao li, Huang yiyu, Zhao yanhong, He ye, Yang jing, Li yixuan, Zhang jiayin, Ma xiao, Yang liang, Bian xia (the Chinese student association in Enschede); Jin mingliang, Shui lingling, Yang jing, Zhao yiping, Zhong zhicheng, Li chen, Zheng ding, Fei teng, Deng dongpo, Hong liang (my Chinese badminton partners); Zhong zhixun, Mei zikai, Yu yizi, Wang xiaojun, Yin zun (the Chinese Bible study group); He yifan, Lu hao, Li xiaomeng, Zhang xiao, Sheng, xiaoqin, Gang tian, Wan xin, Zhang yang, Lu jiwu, Yi jianxin, Qi hong, Shi weiqing, Zhao wei, Ma yujie, Chen qi, Sui xiaofeng, Song jing, Xue yanyan, Lin chao, Jin rong, Li jiajie, Tan jiexian, Wen xinyan etc. thanks for all the nice moments we shared together which made my life in Holland much more colorful.

Last, but not least, I thank my family: my parents, for giving me life, for educating me, and for your unconditional love. All I have achieved today would not be possible without the love from both of you. As an old Chinese saying "with elderly parents at home, a filial son must not travel far", I really appreciate your understanding and support when I studied abroad to pursue my interests. My wife, Qian ying, for being there, supporting me, encouraging me, when writing this thesis.

\section{Xuexin Duan}

Enschede, the Netherlands

January 2010 


\section{Cruuiculum Vitae}

Xuexin Duan was born on the 6th of July 1979, in Tianjin, China. He studied chemistry in Nankai University, China. In the summer of 2000 he entered the State Key Laboratory and Institute of Elemento-organic Chemistry, Nankai University to complete his undergraduate thesis paper. After one year he got his Bachelor degree of Organic Chemistry. In the same year he became a master student in the Institute of Polymer Chemistry in Nankai University, he studied the crystallization behavior of some unique hybrid linear and dendritic block copolymers for his master thesis. In 2004, he received his Master degree of the Chemistry and Physics of Polymer Science. Then he worked one year at Max-Planck Institute for Polymer Research in Mainz, Germany. From December 2005, he was a PhD candidate under the supervision of Prof. Jurriaan Huskens and Prof. David N. Reinhoudt, in the group of Molecular Nanofabrication (MNF) at the University of Twente, the Netherlands, on the project of high resolution soft lithography. The results of his research work are described in this thesis. 



\section{List of publications}

1. X. X. Duan, Y. P. Zhao, A. Perl, E. Berenschot, D. N. Reinhoudt, J. Huskens, "Nanopatterning by an Integrated Process Combining Capillary Force Lithography and Microcontact Printing" Adv. Funct. Mater. 2010, in press

2. X. X. Duan, D. N. Reinhoudt, J. Huskens, "Soft Lithography for Patterning Selfassembling Systems" book chapter of "Functional Supramolecular Architectures for Organic Electronics and Nanotechnology" Ed. Wiley-VCH: Weinheim 2010, in press.

3. X. X. Duan, Y. P. Zhao, A. Perl, E. Berenschot, D. N. Reinhoudt, J. Huskens, "HighResolution Contact Printing with Chemically Patterned Flat Stamps Fabricated by Nanoimprint Lithography" Adv. Mater. 2009, 21, 2798.

4. V. B. Sadhu, A. Perl, X. X. Duan, D. N. Reinhoudt, J. Huskens, "Supramolecular Microcontact Printing with Receptor-functionalized PDMS Stamps” Soft Matter 2009, 5,1198

5. H. P. Xu, X. Y. Ling, J. V. Bennekom, X. X. Duan, M. J. W. Ludden, D. N. Reinhoudt, M. Wessling, R. G. H. Lammertink, J. Huskens "Microcontact Printing of Dendrimers, Proteins, and Nanoparticles by Porous Stamps.” J. Am. Chem. Soc. 2009, 131, 797

6. X. X. Duan, V. B. Sadhu, A. Perl, M. Peter, D. N. Reinhoudt, J. Huskens, "Bifunctional, Chemically Patterned Flat Stamps for Microcontact Printing of Polar Inks" Langmuir 2008, 24, 3621

7. X. X. Duan, F. Yuan, M. Yang, B. L. He, W. Wang, "Alternating CrystallineAmorphous Layers in Hybrid Block Copolymers of Linear Poly(ethylene glycol) and Dendritic Poly(benzyl ether)." Macromol. Chem. Phys. 2004, 205, 1410

8. X. X. Duan, F. Yuan, X. J. Wen, M. Yang, H. Lin, P. C. Sun, B. L. He, W. Wang, "2D NMR Evidence of Formation of Triblock Copolymers of Linear Poly(ethylene glycol) with Two Dendritic Poly(bentzyl ether) Blocks" ) Acta Polym. Sin. 2004, 6, 908

9. Y. B. Zhou, X. X. Duan Q. L. Zhou, "Synthesis of New Chiral Hydrogenated Quinolinyl-Oxazoline Ligands" Chem. Res. Chin. Univ. 2001, 22, 130 


\section{Manuscripts Submitted:}

1. X. X. Duan, Y. P. Zhao, E. Berenschot, N. R. Tas, D. N. Reinhoudt, J. Huskens, "Large-area Nanoscale Patterning of Functional Materials by Nanomolding in Capillaries" submitted

2. M. H. Park, X. X. Duan Y. Ofir, B. Creran, D. Patra, X. Y. Ling, J. Huskens, V. M. Rotello, "Chemically Directed Immobilization of Nanoparticles on Gold Substrates and Orthogonal Assembly using Dithiocarbamate Bond Formationg" submitted

3. X. X. Duan, M. H. Park, Y. P. Zhao, E. Berenschot, Y. Ofir, V. M. Rotello, J. Huskens, "Assembly, Chemical Modification, and Electrical Characterization of Quasi-1D Gold Nanoparticle Arrays via Nanomolding in Capillaries Combined with Dithiocarbamate Bond" in preparation 